\title{
Dynamics and transport in random quantum systems governed by strong-randomness fixed points
}

\author{
Olexei Motrunich, ${ }^{1}$ Kedar Damle, ${ }^{1,2}$ and David A. Huse ${ }^{1}$ \\ ${ }^{1}$ Department of Physics, Princeton University, Princeton, New Jersey 08544 \\ ${ }^{2}$ Department of Electrical Engineering, Princeton University, Princeton, New Jersey 08544 \\ (Received 31 May 2000; revised manuscript received 24 August 2000; published 13 March 2001)
}

\begin{abstract}
We present results on the low-frequency dynamical and transport properties of random quantum systems whose low temperature $(T)$, low-energy behavior is controlled by strong-disorder fixed points. We obtain the momentum- and frequency-dependent dynamic structure factor in the random singlet (RS) phases of both spin-1/2 and spin-1 random antiferromagnetic chains, as well as in the random dimer and Ising antiferromagnetic phases of spin-1/2 random antiferromagnetic chains. We show that the RS phases are unusual "spin metals" with divergent low-frequency spin conductivity at $T=0$, and we also follow the conductivity through "metal-insulator"' transitions tuned by the strength of dimerization or Ising anisotropy in the spin-1/2 case, and by the strength of disorder in the spin-1 case. We work out the average spin and energy autocorrelations in the one-dimensional random transverse-field Ising model in the vicinity of its quantum critical point. All of the above calculations are valid in the frequency-dominated regime $\omega \gtrsim T$, and rely on previously available renormalization group schemes that describe these systems in terms of the properties of certain strong-disorder fixed-point theories. In addition, we obtain some information about the behavior of the dynamic structure factor and dynamical conductivity in the opposite "hydrodynamic" regime $\omega<T$ for the special case of spin-1/2 chains close to the planar limit (the quantum $x-y$ model) by analyzing the corresponding quantities in an equivalent model of spinless fermions with weak repulsive interactions and particle-hole symmetric disorder.

DOI: 10.1103/PhysRevB.63.134424

PACS number(s): 75.10.Jm, 78.70.Nx, 75.50.Ee, 71.30. $+\mathrm{h}$
\end{abstract}

\section{INTRODUCTION}

Disorder effects arising from quenched randomness are at the heart of many interesting and novel phenomena observed in condensed matter systems: examples include Griffiths singularities near phase transitions in disordered magnets (and the related phenomenon of local-moment formation in disordered electronic systems ${ }^{1}$ ), metal-insulator transitions in disordered electronic systems, ${ }^{2}$ and two-dimensional phenomena such as weak localization and the quantum Hall plateau transitions. $^{3}$

In particular, the interplay between disorder and quantum interference leads to unusual dynamics and transport in these systems. Such effects are well understood for disordered quantum systems in which many-body correlations are not significant (such as disordered Fermi liquids). In contrast, relatively little is reliably known about the effects of strong disorder in the presence of strong correlations (say, due to electron-electron interactions in an itinerant electronic system, or due to exchange interactions in a system with localized spin degrees of freedom).

However, there does exist one class of systems where theoretical tools are available to analyze this interplay between strong disorder, correlations, and quantum fluctuations; important examples include one-dimensional random antiferromagnetic spin chains ${ }^{4,5}$ and random quantum Ising models in one and two dimensions. ${ }^{6,7}$ In these quantum systems, it is possible to systematically treat disorder and correlation using a strong-disorder renormalization group (RG) technique that is designed to be accurate when the strength of the disorder, as measured by the widths of the distributions of the various couplings, is large. Such a strongdisorder approach works in these problems because these systems, when studied at ever larger length scales (and cor- respondingly lower energy scales), appear more and more disordered. More precisely, the low-energy effective theory obtained from the RG has the remarkable property that the widths of the distributions of the various couplings in the theory grow rapidly as the energy cutoff is lowered; this means that the RG procedure gives reliable results for the effective Hamiltonian that governs the low-energy properties of the system. Moreover, the extremely strong disorder present at low energies in the effective theory actually allows one to straightforwardly calculate some thermodynamic properties and ground-state correlators within the effective theory-this is, in essence, because strong disorder implies that some particular terms in the effective Hamiltonian dominate over all others; calculations can then be performed by treating these terms first and including the effects of the other terms perturbatively. This approach has been used successfully in the past to obtain a wealth of information about the low-temperature thermodynamics and ground-state correlators in such systems. ${ }^{6,4,8,9,7}$

Here, we exploit this simplicity that emerges at strong disorder to obtain the analytical results on the low-frequency dynamics and transport in these systems at low temperature $T$. Most of our results are obtained for $T=0$; these are expected to be exact at zero temperature in the low-frequency limit, and to remain valid at nonzero temperatures for low frequencies $\omega \gtrsim T$. Moreover, in certain special cases, we can also access the regime $\omega<T$.

In the remainder of this section, we introduce the various systems that are studied in this paper, and describe the organization of the rest of the paper. A brief summary of some of our results has already appeared elsewhere. ${ }^{10}$

Our focus is on three model systems. The first model we consider is the one-dimensional random antiferromagnetic $X X Z$ spin-1/2 chain with the Hamiltonian 


$$
\mathcal{H}_{X X Z}=\sum_{j}\left[J_{j}^{\perp}\left(s_{j}^{x} s_{j+1}^{x}+s_{j}^{y} s_{j+1}^{y}\right)+J_{j}^{z} s_{j}^{z} s_{j+1}^{z}\right],
$$

where $\vec{s}_{j}$ are spin-1/2 operators at lattice sites $j$ separated by spacing $a$, and both $J_{j}^{\perp}$ and $J_{j}^{z}$ are random positive exchange energies drawn from some probability distributions. Such a Hamiltonian describes the low-energy (magnetic) dynamics of insulating antiferromagnetic spin-1/2 chain compounds ${ }^{11,12}$ with chemical disorder that affects the bond strengths. We will also consider chains with slightly different probability distributions of the even and the odd bonds and study the effects of such enforced dimerization. The strength of the dimerization in the bonds is conveniently characterized by a dimensionless parameter $\delta$ defined as

$$
\delta=\frac{\overline{\ln J_{\mathrm{e}}}-\overline{\ln J_{\mathrm{o}}}}{\operatorname{var}\left(\ln J_{\mathrm{e}}\right)+\operatorname{var}\left(\ln J_{\mathrm{o}}\right)},
$$

where $J_{\mathrm{e}}\left(J_{\mathrm{o}}\right)$ represents even (odd) bonds, and the overbar and "var" denote correspondingly the average and variance over the distribution of bonds. Thus, we have $\delta>0(\delta<0)$ if even (odd) bonds are stronger on average. For future reference, we also introduce the basic length scale in this system,

$$
l_{v}=\frac{2 a}{\operatorname{var}\left(\ln J_{\mathrm{e}}\right)+\operatorname{var}\left(\ln J_{\mathrm{o}}\right)} .
$$

Detailed information about the spin dynamics in such systems can be obtained by inelastic neutron scattering (INS) experiments that directly probe the frequency- and momentum-dependent dynamic structure factor $S^{\alpha \beta}(k, \omega)$. At $T=0, S^{\alpha \beta}(k, \omega)$ has the spectral representation

$$
S^{\alpha \beta}(k, \omega)=\frac{1}{L} \sum_{m}\left\langle 0\left|\hat{s}_{-k}^{\alpha}\right| m\right\rangle\left\langle m\left|\hat{s}_{k}^{\beta}\right| 0\right\rangle \delta\left(\omega-E_{m}\right),
$$

where $\hat{s}_{k}^{\alpha}=\Sigma_{j} e^{i k x_{j}} s_{j}^{\alpha}$, and $\{|m\rangle\}$ denote the exact eigenstates of the system with excitation energies $E_{m}$ relative to the ground state $|0\rangle$. The symmetry of $\mathcal{H}_{X X Z}$ under rotations about the $z$ axis implies that we can restrict our attention to two independent components $S^{z z}$ and $S^{+-}$. The same symmetry also implies that the total $s_{\text {tot }}^{z}=\Sigma_{j} s_{j}^{z}$ is conserved-it then makes sense to talk of the spin transport in such a system. We characterize the transport of $s^{z}$ in terms of the dynamical spin conductivity $\sigma(\omega)$. The real part $\sigma^{\prime}(\omega)$ of $\sigma(\omega)$ is defined by the relation $P(\omega)=\sigma^{\prime}(\omega)|\nabla H|^{2}(\omega)$, where $P(\omega)$ is the power absorbed per unit volume by the system when a magnetic field with a uniform gradient $\nabla H(\omega)$ (with the field $H$ always in the $z$ direction) oscillating at frequency $\omega$ is applied along the length of the chain. From standard linear-response theory, we have the following Kubo formula for $\sigma^{\prime}(\omega)$ at $T=0$ :

$$
\sigma^{\prime}(\omega)=\frac{1}{\omega L} \sum_{m}\left|\left\langle m\left|\sum_{j=1}^{L} \tau_{j}\right| 0\right\rangle\right|^{2} \delta\left(\omega-E_{m}\right) .
$$

In the above, $\tau_{j}=i J_{j}^{\perp}\left(s_{j}^{+} s_{j+1}^{-}-s_{j+1}^{+} s_{j}^{-}\right) / 2$ is the current operator on link $j$ that transfers one unit of the $s^{z}$ from one site to the next. Here and everywhere in the following, the fre- quency $\omega$ is taken as positive for notational convenience. Note that both $S^{\alpha \beta}(k, \omega)$ and $\sigma^{\prime}(\omega)$ as defined here are selfaveraging in the thermodynamic limit.

The second model we consider is the random antiferromagnetic Heisenberg spin-1 chain with the Hamiltonian

$$
\mathcal{H}_{\mathrm{S1}}=\sum_{j} J_{j} \vec{S}_{j} \cdot \vec{S}_{j+1}
$$

where $\vec{S}_{j}$ are spin-1 operators on lattice sites $j$, and the $J_{j}$ are random positive nearest-neighbor exchanges; randomness in the system is characterized by a width $W$ of the corresponding distribution of log-exchanges $\ln \left(J_{j}\right)$. As in the spin-1/2 case, we can characterize spin dynamics and transport in terms of the dynamic structure factor and the dynamical conductivity; the definitions remain the same except for the obvious replacement of all spin-1/2 operators with their spin-1 counterparts. Experimental realizations of pure Heisenberg spin-1 chains are known, ${ }^{13}$ and experimental studies of systems with randomness have also been reported in the recent literature. ${ }^{14}$ We caution, however, that the degree of disorder needed to destroy the gapped Haldane phase of a pure spin-1 chain appears to be quite strong, ${ }^{15}$ and that all our calculations are done only in this strong-disorder regime.

The third problem that we consider is the one-dimensional random transverse-field Ising model

$$
\mathcal{H}_{\mathrm{RTFIM}}=-\sum_{j} J_{j} \sigma_{j}^{z} \sigma_{j+1}^{z}-\sum_{j} h_{j} \sigma_{j}^{x},
$$

with random ferromagnetic interactions $J_{j}$ and positive random transverse-fields $h_{j}$; here $\sigma_{j}$ are Pauli spin matrices. The strong-disorder RG approach, and its consequences for the low-temperature thermodynamics and static correlators, have been analyzed in greatest detail for this particular model. ${ }^{16}$ Also, there are extensive numerical results available for some dynamical properties. ${ }^{17}$ This model thus serves as a benchmark to test reliability of our approach to the calculation of dynamical properties in these strong-disorder systems; we will analyze various average autocorrelation functions in considerable detail and compare our results with the earlier numerical work.

The paper is organized as follows: We begin in Sec. II with a general discussion of the various types of states that we encounter in these models, along with an overview of our most important results for the dynamics and transport in various regimes; the last part of this section is devoted to a general outline of the basic approach that is used to obtain these results. Sections III, IV, and V present careful derivations of our results for the zero-temperature dynamical properties of the three model systems that we consider, with each section starting with a review of the basic RG approach used to study the corresponding system. In Sec. III we evaluate the dynamic structure factor and the dynamical conductivity in the various phases of the random $X X Z$ spin-1/2 chain. This is followed, in Sec. IV, by an analysis of the spin conductivity in the strongly-random Heisenberg antiferromagnetic spin-1 chains, and, in Sec. V, by an analysis of the average local dynamical properties of the random quantum 
Ising model in the vicinity of its critical point. Section VI is devoted to a qualitative analysis of the dynamical and transport properties of the $X X Z$ spin-1/2 chains at nonzero temperatures in the regime $\omega<T$, along with some quantitative calculations in the $X X$ spin- $1 / 2$ chain that are possible in this case because of the mapping to free fermions. We conclude, in Sec. VII, with a discussion of the possible experimental tests of some of our predictions for the one-dimensional random-exchange antiferromagnetic spin chains. Some technical details are relegated to the Appendix.

\section{OVERVIEW}

Broadly speaking, our results are for two types of states. First, there are ground states governed (and therefore best described by some suitable strong-disorder RG approach) by infinite-randomness fixed points; examples include the random singlet states of the spin-1/2 antiferromagnetic chains and the critical point of the random transverse-field Ising model. Then, there are the so-called "Griffiths" phases in the immediate vicinity of these critical states; in these phases, the low-energy renormalized randomness is strong, but not infinite.

In both cases the low-energy excitations are localized, but with a characteristic "localization length," i.e., the "size of the excitation," that diverges as a power of $\ln \omega$ for energy $\omega \rightarrow 0$. [We emphasize that this is the statement about the (rare) low-energy excitations and is indeed valid in the Griffiths phases, even though in this case all equal-time correlators at $T=0$ indicate a finite localization length; for details see the main body of the paper.] Apart from this logarithmically divergent "localization length," we can also define, from the integrated density of states $n_{\omega}$ for excitations up to energy $\omega$, a length $L_{\omega} \equiv n_{\omega}^{-1 / d}$ that is the typical spacing between these excitations in $d$ dimensions (the results we report here are for $d=1$, but similar phases do occur ${ }^{7}$ for $d>1$ ).

For a ground state governed by an infinite-randomness fixed point, $L_{\omega}$ diverges at low energies with the same power of $\ln \omega$ as the typical size of the excitation. This means a strongly divergent density of states at low energy, which allows the system to behave as a conductor if there is a conserved quantity (e.g., spin or particle number) to be transported. In a Griffiths phase, on the other hand, $L_{\omega} \sim \omega^{-1 / z}$, with $z$ a nonuniversal dynamical exponent that varies continuously within the phase. Here, the low-energy excitations are rare; they are typically spaced by distance $L_{\omega}$, which diverges as a power-law at low energy and thus is much larger than the excitation's typical size, which is diverging only logarithmically. In the RG language, the Griffiths phases are governed by lines of fixed points ending in the infinite-randomness critical fixed point; along such a line, the dynamical exponent $z$ varies continuously and diverges near the critical point.

In terms of the original microscopic model, the low-lying excitations in the Griffiths phases come from regions where the local-quenched random variables deviate strongly from their global averages. These deviations are such that the local averages would put that region in a different phase. If the system is not at a phase transition, the probability of such a rare region occurring and being of linear size $L$ is $e^{-c_{1} L^{d}}$ for large $L$, for some constant $c_{1}$. Such a rare region typically results in a low-lying mode with a sharply defined (in the sense that $c_{2}$, introduced below, is sharply defined) characteristic frequency proportional to $e^{-c_{2} L^{d}}$. This gives rise to a power-law low-energy density of states, with the dynamical exponent $z$ being determined by the ratio of the constants $c_{1} / c_{2}$. For a disordered Griffiths phase, the rare regions are finite "islands" of either an ordered phase, or a different disordered phase. The resulting low-lying excitations localized on these rare regions produce a low-frequency conductivity $\sigma^{\prime}(\omega)$ or scaled dynamic structure factor $\omega S(k, \omega)$ vanishing as $\omega^{1 / z}$ at low frequencies (apart from possible logarithmic factors attributable to singular low-energy behavior of the relevant matrix elements that may, in some cases, be sensitive to the logarithmically divergent size of the relevant excitations).

For one-dimensional systems, there are also power-law Griffiths effects in Ising-ordered phases. These occur because of rare regions locally in the disordered phase. The low-energy excitation associated with such a region is a domain wall (or "kink"). To produce a single such low-energy domain wall requires flipping the spontaneous magnetization on one side of the the wall, which is tantamount to flipping a semi-infinite piece of the chain. Such a flip of an infinite domain cannot occur at a finite (nonzero) frequency. The leading contribution to the low-frequency dynamics is then associated with two nearby such rare low-energy domain walls that allow the ordered domain between them to flip at a low but nonzero frequency. The result of this is that the low-frequency $\sigma^{\prime}(\omega)$ and $\omega S(k, \omega)$ vanish as $\omega^{2 / z}$ at low frequency in these one-dimensional Ising-ordered Griffiths phases (we are again ignoring possible logarithmic factors that can arise for precisely the same reasons as in the disordered phase). Note, however, that the Griffiths singularities in Ising-ordered phases in $d>1$ are of a very different character; in these cases, the low-energy density of states vanishes faster than any power of $\omega$, as is discussed in Ref. 7.

In Secs. III-V we will provide a detailed justification of these general observations by explicitly calculating the lowfrequency dynamical properties in a variety of cases. In the rest of this section, we review the phase diagrams of our model systems, and highlight our most important results in each case.

\section{A. Random antiferromagnetic $X X Z$ spin-1/2 chains}

\section{Phase diagram}

The phase diagram of the random antiferromagnetic $X X Z$ spin-1/2 chains is best understood as a product of the competition between the transverse part of the coupling $J^{\perp}$, which favors singlet formation, and the "classical" interaction $J^{z}$, which favors a ground state with Ising antiferromagnetic order.

When the $J^{\perp}$ dominate, the ground state can be loosely thought of as being made up of singlet pairs. In this random 


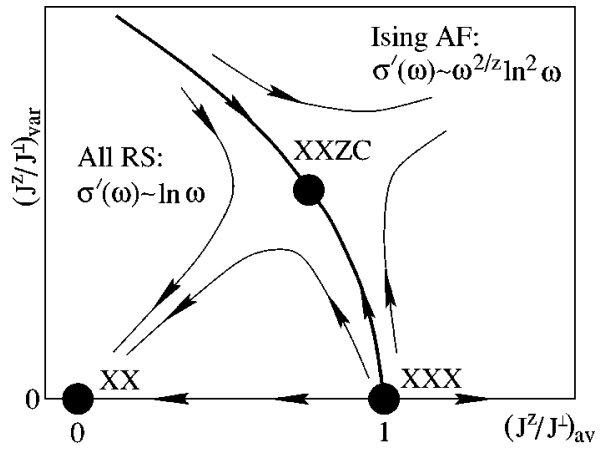

FIG. 1. Schematic phase diagram of random antiferromagnetic $X X Z$ spin-1/2 chains obtained in Ref. 4, showing the three different RS fixed points and RG flows. Our prediction for the low-frequency behavior of the dynamical conductivity is indicated for each phase. For details, see Sec. III; here $z=z\left(\delta_{\mathrm{IAF}}\right)$ is a (continuously varying) dynamical exponent in the IAF Griffiths phase.

singlet (RS) state, the interplay of disorder and quantum fluctuations locks each spin into a singlet pair with another spin; the two spins in a given singlet pair can have arbitrarily large spatial separation, with the disorder determining the particular pattern of the singlet bonds in a given sample. On the other hand, when the $J^{z}$ dominate, the system has Ising antiferromagnetic (IAF) order in the ground state (with the spins all oriented parallel to the $z$ axis), although Griffiths effects can fill in the gap leading to an IAF-ordered Griffiths phase.

These two states are separated by a quantum phase transition that occurs when the couplings $J^{\perp}$ and $J^{z}$ have roughly similar distributions (have roughly equal strengths). A special feature of this system is that the ground state at any point on the critical manifold is also a random singlet state, though the details of the excitation spectrum are somewhat different.

If we now turn on enforced bond dimerization starting with the RS state that obtains for small $J^{z}$, or the RS state of the Heisenberg chain, the system moves into a Griffiths phase dubbed the random dimer (RD) phase; in this phase the singlet bonds in the ground state now preferentially start on one sublattice and end on the other.

Schematic phase diagrams summarizing the above are shown in Figs. 1 and 2.

\section{Spin transport}

We characterize the spin transport properties of the various phases in terms of the low-frequency behavior of the dynamical conductivity: We find that the $T=0$ dynamical conductivity diverges at low frequencies in the RS phase as well as at the RS critical points as

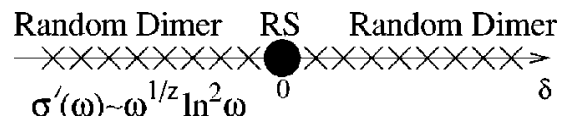

FIG. 2. The random dimer phases for $X X$ or Heisenberg spin$1 / 2$ antiferromagnetic chains, represented as lines of fixed points ending in the critical fixed point labeled RS that describes the random singlet state at zero dimerization (Ref. 9); here $z=z(\delta)$ is a dynamical exponent in the RD Griffiths phase.

$$
\sigma^{\prime}(\omega)=\mathcal{K}_{\mathrm{RS}} l_{v} \Gamma_{\omega},
$$

where we have taken the opportunity to introduce the logenergy scale

$$
\Gamma_{\omega} \equiv \ln \left(\Omega_{0} / \omega\right) .
$$

Here and henceforth we use $\Omega_{0}$ to denote the nonuniversal microscopic energy cutoff, which corresponds roughly to the energy scale in the bare Hamiltonian for our various models; also, we use $l_{v}$ to denote the nonuniversal microscopic length scale in the problem. For the $X X Z$ spin-1/2 system near the RS phase and with sufficiently strong disorder, which is what we assume in the following, the microscopic length $l_{v}$ is given by Eq. (3). (If, on the other hand, the bare disorder is weak and the system flows to strong disorder, then $l_{v}$ is the length scale at which the strength of the disorder becomes of order one.) $\mathcal{K}_{\mathrm{RS}}$ in Eq. (8) is an order-one numerical constant. The RS phase and the RS critical points separating it from the IAF phase are thus unusual spin conductors.

On the other hand, the IAF Griffiths phase is a spin insulator with the low-frequency $T=0$ dynamical conductivity

$$
\sigma^{\prime}(\omega)=\mathcal{K}_{\mathrm{IAF}} l_{v}\left(\omega / \Omega_{0}\right)^{2 / z} \operatorname{IAF} \ln ^{2}\left(\Omega_{0} / \omega\right),
$$

where $z_{\mathrm{IAF}}\left(\delta_{\mathrm{IAF}}\right)$ is a (continuously varying) dynamical exponent diverging at the critical point as $z_{\mathrm{IAF}} \sim \delta_{\mathrm{IAF}}^{-(2-\psi) / \lambda}$, and $\mathcal{K}_{\text {IAF }}$ is a nonuniversal amplitude vanishing at the transition as $\mathcal{K}_{\mathrm{IAF}} \sim \delta_{\mathrm{IAF}}^{(2-\psi) / \lambda}$. Here we parametrized the distance from the transition to the RS phase by $\delta_{\mathrm{IAF}} \equiv \bar{\Delta}-\bar{\Delta}_{c}$ (where $\Delta$ $\equiv J^{z} / J^{\perp}$ ). The exponent $\lambda$ is the relevant RG eigenvalue controlling the flow away from the critical fixed point describing the generic transition between the RS phase and the IAF phase, and the exponent $\psi$ characterizes the low-energy spectrum above the RS ground state at this critical point (see Ref. 4 and Sec. III A for details). The above result is expected to hold in the frequency regime $\omega \ll \Omega_{\delta_{\mathrm{IAF}}}$ with the crossover scale $\Omega_{\delta_{\mathrm{IAF}}}$ given in terms of $\delta_{\mathrm{IAF}}$ as $\ln \left(\Omega_{0} / \Omega_{\delta_{\mathrm{IAF}}}\right) \sim \delta_{\mathrm{IAF}}^{-(2-\psi) / \lambda}$.

Similarly, the RD phases are also spin insulators, with the $T=0$ low-frequency dynamical conductivity

$$
\sigma^{\prime}(\omega)=\mathcal{K}_{\mathrm{RD}} l_{v}\left(\omega / \Omega_{0}\right)^{1 / z_{\mathrm{RD}} \ln ^{2}\left(\Omega_{0} / \omega\right) ;}
$$

the dynamical exponent $z_{\mathrm{RD}}(\delta)$ in the RD phase diverges at the transition as $z_{\mathrm{RD}} \sim|\delta|^{-1}$, and the nonuniversal amplitude $\mathcal{K}_{\mathrm{RD}}$ vanishes at the transition as $\mathcal{K}_{\mathrm{RD}} \sim|\delta|$. As in the IAF phase, this result is valid at frequencies well below the corresponding crossover scale $\Omega_{\delta}$ (which can be also viewed as the conductivity pseudogap scale); in the RD phases $\ln \left(\Omega_{0} / \Omega_{\delta}\right) \sim 1 /|\delta|$.

Thus, in both the IAF phase and the RD phase, the conductivity has the functional form

$$
\sigma^{\prime}(\omega) \sim \omega^{\alpha} \ln ^{2} \omega,
$$

with the nonuniversal exponent $\alpha$ vanishing at the corresponding transition. Note that a similar form but with fixed $\alpha=2$ - the Mott formula-is obtained via the usual Mott argument for the $T=0$ dynamical conductivity of the onedimensional Anderson insulator (the fixed value of $\alpha$ in this 


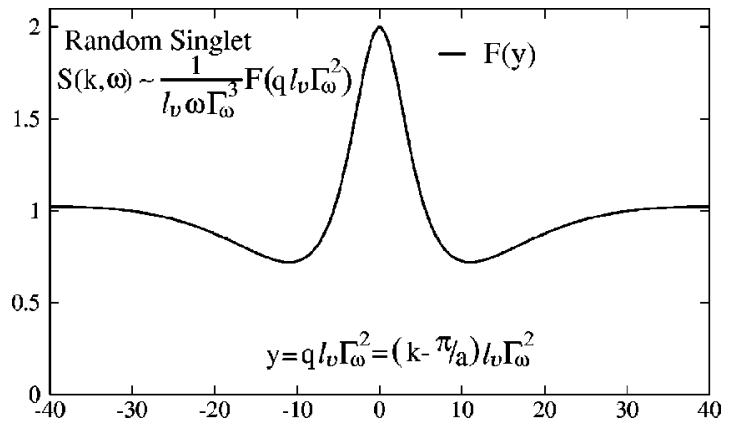

FIG. 3. Sketch of the dynamic structure factor at fixed $\omega \ll \Omega_{0}$ in the RS states.

case simply reflects the fact that low-energy density of states in the Anderson insulator is constant, in contrast to the situation in the Griffiths phases of interest to us here).

\section{Spin dynamics}

Turning to the spin dynamics, we find that the $T=0 \mathrm{dy}-$ namic structure factor in the RS states in the vicinity of $k$ $=\pi / a$ can be written in the following unusual scaling form

$$
S^{\alpha \beta}\left(k=\frac{\pi}{a}+q, \omega\right)=\frac{\mathcal{A}}{l_{v} \omega \ln ^{3}\left(\Omega_{0} / \omega\right)} \Phi\left[\left|q l_{v}\right|^{1 / 2} \ln \left(\Omega_{0} / \omega\right)\right]
$$

for $|q| \ll a^{-1}$ and $\omega \ll \Omega_{0}$; here $\alpha \beta \equiv+-$ or $z z, \mathcal{A}$ is an order-one numerical constant, $l_{v}$ is the microscopic length defined earlier, and $\Phi(x)$ is the fully universal function explicitly calculated in Sec. III. A plot of the momentum dependence of the dynamic structure factor near $k=\pi / a$ (at fixed low frequency) is shown in Fig. 3; an interesting aspect is the nonmonotonic nature of the line shape. We will see in Sec. III that this oscillatory behavior becomes more pronounced and leads to a really striking structure in the momentum dependence of the dynamic structure factor at (fixed) low frequency $\omega \ll \Omega_{\delta}$ in the random dimer phases; a plot of the expected $k$ dependence is shown in Fig. 4. A very similar dependence is also predicted in the IAF Griffiths phase close to the transition to the RS state.

As mentioned earlier, these results are expected to remain valid at small nonzero temperatures so long as the frequency

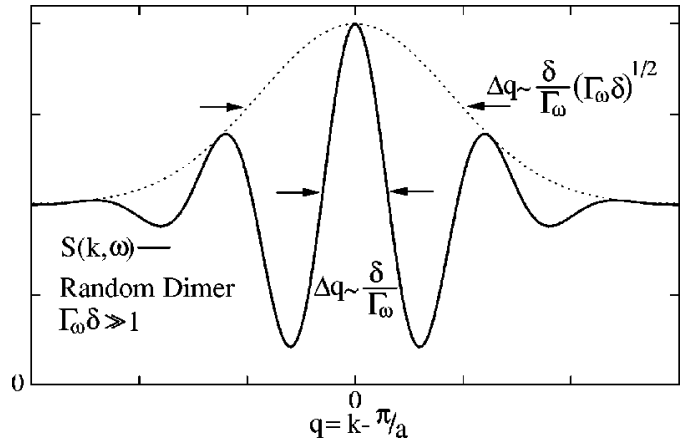

FIG. 4. Sketch of the dynamic structure factor at fixed $\omega \ll \Omega_{\delta}$ in the RD phases.

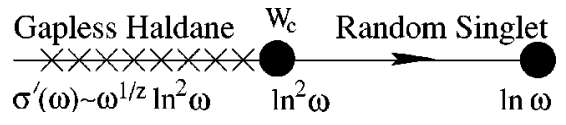

FIG. 5. Schematic phase diagram of the strongly disordered Heisenberg spin-1 chain (Ref. 5), along with our results for the dynamical conductivity in various regimes. Moving to the right along the horizontal axis corresponds to increasing disorder.

$\omega$ satisfies $\omega \gtrsim T$. In Sec. VI, we will see that we can partially overcome even this restriction in the vicinity of the $X X$ point.

\section{B. Spin-1 Heisenberg antiferromagnetic chains}

\section{Phases}

The effect of randomness on antiferromagnetic Heisenberg spin-1 chains is even more interesting. For spin-1, there are, in general three distinct phases possible in the presence of disorder. If the disorder is weak, and the support of the probability distribution $P(J)$ of the exchanges is confined to a narrow-enough region near the mean, then the system remains in the usual gapped, topologically ordered Haldane state. For stronger disorder, or when $P(J)$ has tails to large or small enough $J$, one has the "gapless Haldane", (GH) phase in which the system still has the topological order that characterizes the Haldane state, but becomes gapless due to Griffiths effects. Finally, if the disorder is extremely strong, with the (bare) distribution of exchanges broad on a logarithmic scale, a random singlet state completely analogous to the one encountered in the spin-1/2 case is obtained. While the GH state and the RS state are separated by a quantum critical point with universal critical properties (these properties are in fact controlled by a strong-disorder fixed point ${ }^{5,8}$ ), the corresponding transition between the gapped and gapless Haldane states is a nonuniversal feature of the phase diagram, depending sensitively on the nature of the initial distribution of couplings (see Fig. 5 for a summary of the universal aspects of the phase diagram).

\section{Overview of results}

In the spin-1 RS state, we obtain the same results for the dynamic structure factor and spin conductivity as in the spin$1 / 2$ RS state, as the low-energy behavior of the RS state does not depend on the spin magnitude except through the values of some microscopic scale factors. Unfortunately, once we move away from the random singlet state, it is difficult to discuss reliably the momentum dependence of the dynamic structure factor of the original spin-1 chain, because our actual calculations are done in an effective model (see Sec. IV A and Refs. 5 and 8 for details) in which much of the spatial information about the original system is missing.

However, it is still possible to calculate transport properties, such as the dynamical conductivity, that are insensitive to the details of the spatial structure (this is, in essence, a consequence of spin conservation). At the critical point separating the gapless Haldane state from the random singlet state, we find for $\omega \ll \Omega_{0}$ 


$$
\sigma^{\prime}(\omega)=\mathcal{K}_{\mathrm{HY}} l_{v} \ln ^{2}\left(\Omega_{0} / \omega\right),
$$

which is a stronger divergence than in the strong-disorder $\mathrm{RS}$ phase; here $l_{v}$ is the nonuniversal microscopic length scale beyond which the effective model applies, $\Omega_{0}$ is the corresponding microscopic energy scale, and $\mathcal{K}_{\mathrm{HY}}$ is an order-one numerical constant. Thus, the critical point separating the RS phase from the $\mathrm{GH}$ phase is also an unconventional "spin metal." The GH phase, on the other hand, is a "'spin insulator," not unlike the RD phase of spin-1/2 chains. We find for the conductivity in the $\mathrm{GH}$ phase

$$
\sigma^{\prime}(\omega) \sim \mathcal{K}_{\mathrm{GH}} l_{v}\left(\omega / \Omega_{0}\right)^{1 / z_{\mathrm{GH}}} \ln ^{2}\left(\Omega_{0} / \omega\right) .
$$

The dynamical exponent $z_{\mathrm{GH}}$ varies continuously in the gapless Haldane phase, diverging at the critical point as $z_{\mathrm{GH}}$ $\sim\left(W_{c}-W\right)^{-\nu / 3}$, while the nonuniversal amplitude $\mathcal{K}_{\mathrm{GH}}(W)$ remains nonzero as one approaches the critical point. In the above, $W_{c}$ is the critical value of the bare disorder (the parameter $W$ has already been defined in Sec. I), and the correlation length exponent $\nu=6 /(\sqrt{13}-1)$ is known from the analyses in Refs. 5 and 18.

\section{Random quantum Ising spin chains}

\section{Phases}

The self-dual nature of the random transverse field Ising model in one dimension implies that the system will be in a critical state if the distributions of bonds and fields are identical. The deviation from criticality may be parametrized by

$$
\delta=\frac{\overline{\ln h}-\overline{\ln J}}{\operatorname{var}(\ln h)+\operatorname{var}(\ln J)},
$$

with $\delta>0$ corresponding to the quantum disordered paramagnet, and $\delta<0$ corresponding to the ordered ferromagnet. (Note that we use " $\delta$ " ' both as a dimensionless measure of dimerization in spin-1/2 chains, and in the present context; there is however no cause for confusion and the meaning will always be clear from the context in what follows.)

This quantum critical point is flanked, for small $|\delta|$ on either side, by paramagnetic and ferromagnetic Griffiths phases with gapless excitations.

\section{Overview of results}

As mentioned earlier, these Griffiths phases and the quantum critical point separating them are among the bestunderstood examples of such strong-randomness phenomena. However, all previous analyses of the dynamical properties relied on numerical results supplemented by scaling ideas.

In contrast, our approach allows us to analytically calculate the average local autocorrelations of both the spin and the energy operators at, and in the vicinity of, the quantum critical point, as well as obtain the scaling behavior of the dynamic structure factor of the spins. The main features of the average autocorrelations (as well as distributions of autocorrelations, which we do not address here) have already been noted in the earlier numerical work (Ref. 17), while our results on the dynamical structure factor are new. Here, we only highlight some of the subtleties, missed in these numerical studies, that our analytical work has uncovered regarding the autocorrelations - a complete tabulation of our predictions (and their interpretation in terms of Griffiths effects) is given in Sec. V.

Our results for the $T=0$ imaginary-time off-critical spin autocorrelation in the bulk have the form

$$
\left[C_{\mathrm{loc}}\right]_{\mathrm{av}}(\tau) \sim \frac{|\ln \tau|}{\tau^{n / z(\delta)}},
$$

where $z(\delta)$ is the continuously varying dynamical exponent characterizing the Griffiths phases (from the results of Ref. 6, $z^{-1} \approx 2|\delta|$, for small enough $\delta$ ). In the above, the parameter $n$ distinguishes between the disordered and ordered phases with $n=1$ in the disordered phase and $n=2$ in the ordered phase. Thus, the exponent controlling the power-law decay in the ordered Griffiths phase is twice $z^{-1}$, while the corresponding exponent in the paramagnetic Griffiths phase is $z^{-1}$. This reflects the physical distinction between the disordered and the Ising ordered Griffiths phases noted in our general discussion at the beginning of this overview. Moreover, the autocorrelations in the Griffiths phases are not pure power law, but have a logarithmic correction, which reflects the fact that the appropriate "spin" degrees of freedom relevant at a time-scale $\tau$ have an effective moment of order $|\ln \tau|$. Both these subtleties have been ignored when extracting the dynamical exponent from the numerical results for the average spin autocorrelations via the ansatz $\left[C_{\mathrm{loc}}\right]_{\mathrm{av}}(\tau)$ $\sim 1 / \tau^{1 / z(\delta)}$, and this could account for some of the discrepancies observed in the numerical studies. Similar remarks apply to other average autocorrelations considered, and we refer to Sec. V for details.

\section{The basic strategy}

We conclude with an overview of the basic strategy introduced by us in Ref. 10 for the calculation of dynamical and transport properties-we will be using this approach over and over again in what follows, and while the details will differ from calculation to calculation, the basic approach will remain unchanged.

Consider, for concreteness, the calculation of the dynamic structure factor $S^{\alpha \beta}(k, \omega)$ for the Hamiltonian $\mathcal{H}_{X X Z}$. The basic idea is to eliminate high-energy degrees of freedom using an appropriate strong-disorder renormalization group procedure (in this case, the singlet RG reviewed in Sec. III A), and trade in the spectral sum Eq. (4) for a sum over the eigenstates of the renormalized Hamiltonian $\tilde{\mathcal{H}}_{X X Z}$, which has fewer degrees of freedom and renormalized bond strengths. This renormalized spectral sum must use the matrix elements of the renormalized versions of the spin operators; these renormalized operators are of course defined by the requirement that their matrix elements between the eigenstates of the renormalized problem reproduce the matrix elements of the original operators between the corresponding eigenstates of the original problem. In the systems of interest to us, the low-energy renormalized randomness is very large. In the renormalized problem at the energy cutoff 
$\Omega \ll \Omega_{0}$, the effective bonds thus have a very broad distribution characteristic of the fixed point to which the system flows in the low energy limit. This allows us to reason as follows: Focus on pairs of spins coupled by "strong" bonds in the renormalized problem, with strengths equal to the cutoff $\Omega$. The broad distribution of bonds implies that these pairs are effectively isolated from their neighbors. It is therefore possible to unambiguously identify the excited states of these pairs with excitations of the full system at the same energies and work out the matrix elements connecting these to the ground state using the renormalized operators. Thus, to calculate the spectral sum Eq. (4), the RG is run till the cutoff $\Omega$ equals $\Omega_{\text {final }}$, and the problem is reduced to calculating the renormalized spectral sum in this new theory; $\Omega_{\text {final }}$ is chosen so that the energy of such excited states (associated with these strong bonds) measured from the ground state equals $\omega$. The calculation of $S^{\alpha \beta}(k, \omega)$ then becomes a counting problem. One uses the known statistical properties of the renormalized bonds in the theory with cutoff $\Omega_{\text {final }}$ to calculate the number of such strong bonds, and simply adds up their contributions weighted by the corresponding matrix elements to obtain the required result. This result is expected to be asymptotically accurate in the limit of small $\omega$, since these contributions clearly dominate in the low-frequency limit. A certain simplicity thus emerges when the low-energy effective theory has strong disorder, and we will exploit this to the fullest in what follows.

\section{DYNAMICS AND TRANSPORT IN THE $s=1 / 2 \quad X X Z$ CHAINS}

\section{A. Detailed characterization of the phases}

\section{Singlet $R G$ description of the random singlet states: A review}

We begin by noting that the weak-randomness analysis of Doty and Fisher ${ }^{19}$ implies that randomness is relevant for pure antiferromagnetic $X X Z$ spin-1/2 chains for $0 \leqslant J^{z} / J^{\perp}$ $\leqslant 1$; any amount of randomness is thus expected to drive the system to strong disorder in this entire regime.

In the strong-disorder regime, the singlet $\mathrm{RG}$ proceeds as follows: ${ }^{20,4}$ We look for the bond with the largest $J^{\perp}$ coupling, say $J_{23}^{\perp}$ between spins 2 and 3; this sets the energy cutoff $\Omega \equiv \max \left\{J_{j}^{\perp}\right\}$. We first solve the corresponding twospin problem and introduce the neighboring bonds later as a perturbation. So long as the $J^{z}$ couplings are not large compared to the $J^{\perp}$ couplings, the ground state of the two-spin problem will always be a singlet separated by a large gap from the triplet excited states. We can then trade our original Hamiltonian in for another Hamiltonian (determined perturbatively in the ratio of the neighboring bonds to the strongest bond) that acts on a truncated Hilbert space with the two sites connected by the "strong" bond removed. To leading order, this procedure renormalizes the Hamiltonian

$$
\mathcal{H}_{4 \mathrm{sites}}=\sum_{j=1}^{3}\left[J_{j}^{\perp}\left(s_{j}^{x} s_{j+1}^{x}+s_{j}^{y} s_{j+1}^{y}\right)+J_{j}^{z} s_{j}^{z} s_{j+1}^{z}\right]
$$

to

$$
\widetilde{\mathcal{H}}_{14}=\widetilde{J}_{1}^{\perp}\left(s_{1}^{x} s_{4}^{x}+s_{1}^{y} s_{4}^{y}\right)+\widetilde{J}_{1}^{z} s_{1}^{z} s_{4}^{z}
$$

with $\widetilde{J}_{1}^{\perp}=J_{1}^{\perp} J_{3}^{\perp} /\left(J_{2}^{\perp}+J_{2}^{z}\right)$ and $\widetilde{J}_{1}^{z}=J_{1}^{z} J_{3}^{z} / 2 J_{2}^{\perp}$; note that the length of this new bond is $\widetilde{l}_{1}=l_{1}+l_{2}+l_{3}$. This procedure, if it remains valid upon iteration, thus ultimately leads to the random singlet state described in the overview.

A complete understanding of the possible phases then requires an analysis of the effects of iterating the basic RG procedure. Such an analysis was performed in Ref. 4 leading to the following conclusions (see Fig. 1): So long as the $J^{z}$ couplings do not dominate over the $J^{\perp}$ couplings and therefore do not produce a state with Ising antiferromagnetic order, the ground state is a random singlet state. In this case, a detailed characterization of the low-energy effective Hamiltonian is best couched in terms of logarithmic variables as follows: Let $\Omega \equiv \max \left\{J_{j}^{\perp}\right\}$ at any given stage of the RG, and define the $\log$-cutoff $\Gamma \equiv \ln \left(\Omega_{0} / \Omega\right)$. Also define $\log$ couplings $\zeta_{j} \equiv \ln \left(\Omega / J_{j}^{\perp}\right)$ and log-anisotropy parameters $D_{j}$ $\equiv \ln \left(\Delta_{j}\right)$, where $\Delta_{j} \equiv J_{j}^{z} / J_{j}^{\perp}$. As $\Gamma$ increases, the fraction of remaining sites $n_{\Gamma}$ at log-cutoff scale $\Gamma$ is given as $n_{\Gamma}$ $\sim 1 / \Gamma^{2}$. When the $J^{\perp}$ couplings dominate, the system rapidly flows to the " $X X$-RS" fixed point and the probability distribution $P(\zeta, \Delta, l \mid \Gamma)$ that determines the strengths and lengths of the bonds connecting the remaining sites in the effective Hamiltonian quickly converges to the following scaling form characteristic of the $X X$-RS fixed point: $P(\zeta, \Delta, l \mid \Gamma)$ $=\left(1 / \Gamma^{3}\right) \mathcal{P}_{1}\left(\zeta / \Gamma, l / \Gamma^{2}\right) \times \delta(\Delta)$. The function $\mathcal{P}_{1}$ has been characterized in detail in Ref. 4; here we only note that $\int d y \mathcal{P}_{1}(x, y)=e^{-x}$. Between the IAF phase and this $X X$-RS phase lie two kinds of critical points. If the initial problem has full Heisenberg symmetry $\left(J^{z}=J^{\perp}\right.$ for each bond), the low-energy effective Hamiltonian preserves this symmetry and has bond strengths and lengths drawn from the same probability distribution: $P(\zeta, l \mid \Gamma)=\left(1 / \Gamma^{3}\right) \mathcal{P}_{1}\left(\zeta / \Gamma, l / \Gamma^{2}\right)$. In the RG language, the Heisenberg system is critical and is controlled by the " $X X X$-RS" critical fixed point. Finally, in this language, the generic critical point between the IAF phase and the $X X$-RS phase is controlled by the " $X X Z C$-RS' fixed point- the low-energy effective theory has bond strengths and lengths drawn from a distribution $P(\zeta, D, l \mid \Gamma)=\left(1 / \Gamma^{3+\psi}\right) \mathcal{P}_{2}\left(\zeta / \Gamma, D / \Gamma^{\psi}, l / \Gamma^{2}\right)$ with $\psi<1$ and $\int d y \mathcal{P}_{2}(x, y, z)=\mathcal{P}_{1}(x, z)$. Notice that these scaling forms imply that the distributions of the couplings become infinitely broad as $\Omega \rightarrow 0$; thus, the RG becomes asymptotically exact at low energies and, in particular, predicts the ground-state properties and low-temperature thermodynamics correctly.

\section{Scaling description of the Ising antiferromagnet}

On the Ising antiferromagnet side, the singlet RG becomes invalid at low energies, and the system has a ground state with IAF order. The proper characterization of the system at these low energies is in terms of IAF-ordered spin clusters, as well as the domain-wall excitations that act to disrupt this order. This section is devoted to providing such a description. In what follows, we will be considering mainly the IAF phase close to the transition to the RS state. In this regime, the system will "look" IAF ordered only well below a crossover energy $\Omega_{\delta_{\mathrm{IAF}}}$, while resembling a critical system 
controlled by the $X X Z C$ critical point above the crossover scale. $\Omega_{\delta_{\text {IAF }}}$ is the scale at which the singlet RG breaks down and is thus determined by the properties of the RG flows in the vicinity of the $X X Z C$ critical point. The corresponding log-energy scale $\Gamma_{\delta_{\text {IAF }}} \equiv \ln \left(\Omega_{0} / \Omega_{\delta_{\text {IAF }}}\right)$ is given as ${ }^{4} \Gamma_{\delta_{\text {IAF }}}$ $\sim \delta_{\mathrm{IAF}}^{-\theta}$, with $\theta=(2-\psi) / \lambda$, where $\lambda$ is the leading relevant RG eigenvalue at the $X X Z C$ fixed point and $\psi$ has already been defined in the previous section. Below, we construct a scaling description of the IAF phase near criticality by combining information obtained from the singlet RG about the nature of the system at this crossover scale, with a "cluster RG" approach that is designed to work in the limit of low energies (well below $\Omega_{\delta_{\mathrm{IAF}}}$ ) above the IAF-ordered ground state.

We begin with a sketch of our cluster RG approach. Consider the Hamiltonian $\mathcal{H}_{X X Z}$ with $J^{z}$ couplings completely dominating the $J^{\perp}$ couplings. Now, spins tend to order antiferromagnetically, and we can try formulating a cluster RG similar to that for the ordered phase of the random transverse-field Ising model. Consider combining two such spins, say $s_{2}$ and $s_{3}$, coupled by a strong bond $J_{2}^{z}$ into a new "superspin" $\tilde{s}_{(23)}$. If we identify the two states $\left|\Uparrow_{(23)}\right\rangle$ and $\left|\Downarrow_{(23)}\right\rangle$ of this superspin with the states $\left|\uparrow_{2} \downarrow_{3}\right\rangle$ and $\left|\downarrow_{2} \uparrow_{3}\right\rangle$ (which is not a unique choice), and treat the $J^{\perp}$ couplings to second order in perturbation theory, the effective Hamiltonian that we obtain is

$$
\begin{aligned}
\widetilde{\mathcal{H}}_{1(23) 4}= & \widetilde{J}_{1}^{z} s_{1}^{z} \tilde{s}_{(23)}^{z}-\widetilde{J}_{3}^{z} s_{4}^{z} \tilde{s}_{(23)}^{z}+\widetilde{h}_{(23)} \widetilde{s}_{(23)}^{x}- \\
& -\widetilde{J}_{1(23) 4}^{\perp}\left(s_{1}^{+} \tilde{s}_{(23)}^{-} s_{4}^{-}+s_{1}^{-} \tilde{s}_{(23)}^{+} s_{4}^{+}\right),
\end{aligned}
$$

where $\widetilde{h}_{(23)}=J_{2}^{\perp}, \widetilde{J}_{1(23) 4}^{\perp}=J_{1}^{\perp} J_{3}^{\perp} / J_{2}^{z}, \widetilde{J}_{1}^{z}=J_{1}^{z}+\left(J_{1}^{\perp}\right)^{2} / J_{2}^{z}$, and $\widetilde{J}_{3}^{z}=J_{3}^{z}+\left(J_{3}^{\perp}\right)^{2} / J_{2}^{z}$. Thus, we see that new terms, not present in the original Hamiltonian, are generated: an effective transverse field, which acts to flip the new spin, and also a threespin exchange interaction. Before we proceed, a couple of comments regarding the new terms: The effective transverse field appears because the ground state of $\mathcal{H}_{23}$ is not exactly a degenerate doublet (the two lowest eigenstates, which are the symmetric and antisymmetric combinations of $\left|\uparrow_{2} \downarrow_{3}\right\rangle$ and $\left|\downarrow_{2} \uparrow_{3}\right\rangle$, are actually split by a small energy $J_{2}^{\perp}$ ). Note also that the three-spin term does not violate spin conservation; for example, if we consider coupling the conserved total $s_{\text {tot }}^{z}$ to a magnetic field, we immediately realize that the superspin $\tilde{s}_{(23)}$ does not couple to this field.

In principle, we may proceed with such a clustering process, keeping track of all additional one- or two- or multispin-flip terms that are generated. While this RG is not analytically tractable, we do not expect the generated terms to have any drastic consequences, since they generally become weaker and weaker, while the $J^{z}$ couplings remain almost unchanged. Alternatively, we can remedy this proliferation of new couplings by combining an odd number of spins at a time-because of the symmetries of the Hamiltonian, any odd length chain will have a degenerate pair of ground states with the total $s_{\text {tot }}^{z}= \pm \frac{1}{2}$. In addition, three-spin terms of the form encountered previously will now be forbidden by spin conservation. More explicitly, if we combine three spins, say $s_{2}, s_{3}$, and $s_{4}$, with relatively strong couplings $J_{2}^{z}$ and $J_{3}^{z}$, into a new superspin $\tilde{s}_{2} \equiv \tilde{s}_{(234)}$, and treat the $J^{\perp}$ couplings perturbatively, the $X X Z$ form of the effective Hamiltonian is preserved, with the new couplings $\widetilde{J}_{12}^{z}=J_{1}^{z}+\left(J_{1}^{\perp}\right)^{2} /\left(2 J_{2}^{z}\right)$, $\widetilde{J}_{25}^{z}=J_{4}^{z}+\left(J_{4}^{\perp}\right)^{2} /\left(2 J_{3}^{z}\right), \quad \widetilde{J}_{12}^{\perp}=-2 J_{1}^{\perp} J_{3}^{\perp} / J_{2}^{z}, \quad$ and $\quad \widetilde{J}_{25}^{\perp}$ $=-2 J_{2}^{\perp} J_{4}^{\perp} / J_{3}^{z}$.

Either way, we will have effective spin-half objects with dominant Ising AF interactions. Almost always, we will be decimating strong $J^{z}$ couplings making larger and larger clusters, with the other $J^{z}$ couplings remaining essentially unchanged, and the remaining $J^{\perp}$ couplings growing weaker and weaker. Only rarely will there be a bond with a $J^{\perp}$ coupling large compared to the neighboring couplings, and this will then produce a singlet. Thus, the picture that emerges is very reminiscent of the ordered phase in the RTFIM.

We may now combine this schematic cluster RG description valid at low energies, with information about the crossover region obtainable from the singlet RG. At the crossover scale, the distribution of $\zeta^{z} \equiv \ln \left(\Omega_{\delta_{\mathrm{IAF}}} / J^{z}\right)$ is given as $P\left(\zeta^{z} \mid \Gamma_{\delta_{\mathrm{IAF}}}\right) \sim \Gamma_{\delta_{\mathrm{IAF}}}^{-1} \exp \left(-\zeta^{z} / \Gamma_{\delta_{\mathrm{IAF}}}\right)$. Roughly speaking, beyond the crossover scale, the cluster RG merely eliminates the strongest bonds from this distribution, but keeps the lowenergy tail of the distribution unchanged. We thus expect a line of (classical) IAF fixed points, with properties varying smoothly with the distance from the criticality. The density of spin degrees of freedom $n_{\Gamma}$ in the renormalized theory is expected to decrease as $n_{\Gamma} \sim \Gamma_{\delta_{\mathrm{IAF}}}^{-2} e^{-c \Gamma / \Gamma} \delta_{\delta_{\mathrm{IAF}}}$ below the crossover scale $\Omega_{\delta_{\text {IAF }}}$, where $c$ is some order-one constant. This immediately gives us the density of states $\rho(\omega)$ $\sim \omega^{-1} n_{\Gamma_{\omega}} \Gamma_{\delta_{\mathrm{IAF}}}^{-1} \sim \delta_{\mathrm{IAF}}^{3 \theta} \omega^{-1+1 / z_{\mathrm{IAF}}}$, with the continuously varying dynamical exponent $z_{\mathrm{IAF}} \sim \delta_{\mathrm{IAF}}^{-\theta}$. The typical size of the excitations dominating the density of states scales as $l_{\mathrm{dw}}(\omega) \sim l_{v} \Gamma_{\delta_{\mathrm{IAF}}} \Gamma_{\omega}$ and is much smaller than their typical

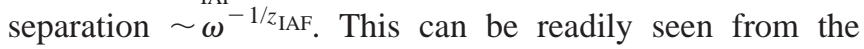
qualitative picture of "preformed tails:" the length $l_{\mathrm{dw}}(\omega)$ of a renormalized bond with $\zeta^{z}=0$ in the theory with cutoff $\omega \ll \Omega_{\delta_{\text {IAF }}}$ scales in the same way as the length of a bond with $\zeta^{z} \sim \ln \left(\Omega_{\delta_{\text {IAF }}} / \omega\right)$ in the theory at the crossover scale $\Omega_{\delta_{\mathrm{IAF}}}$. On the other hand, the distribution of the logcouplings $\zeta^{\perp} \equiv \ln \left(\Omega / J^{\perp}\right)$ is expected to broaden exponentially as a function of $\Gamma$ : for example, when we combine $n$ spins that are active at the crossover scale into a cluster, the effective transverse coupling acting on this cluster will be of order

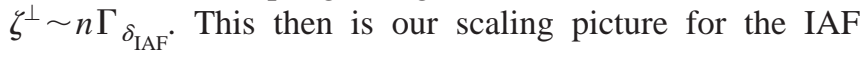
phase; the important conclusion that emerges from this analysis is the fact that the transition to the RS state is preceded by a Griffiths phase- - the IAF Griffiths phase-with a continuously varying power-law singularity in the lowenergy density of states.

Finally, it is also possible to obtain a rather direct identification of the low-energy modes in terms of the rare regions that dominate the low-energy dynamics; we conclude by sketching this briefly here. The RG picture suggests that in 
the IAF phase the typical excitations at low energies $\omega$ are classical domain-wall excitations that live on the effective bonds with weak effective couplings $\widetilde{J}^{z} \sim \omega$. Such a weak effective $\widetilde{J}^{z}$ can appear only across a long region that is locally in the RS phase. More quantitatively, a region of length $L$ locally in the RS phase effectively corresponds to a weak bond with $\widetilde{J}^{z} \sim \Omega_{0} e^{-c_{z} L}$. The number density of such regions is roughly $\sim p^{L}$ for some $p<1$. The density of such regions with $\widetilde{J}^{z} \lesssim \omega$ is thus some power of $\omega$ that we choose to write as $n(\omega) \sim \omega^{1 / z}$ with some exponent $z$; the most numerous such regions will have some "optimal" (for a given $\left.\delta_{\mathrm{IAF}}\right)$ microscopic structure, but whatever this structure is, the corresponding optimal exponent $z$ can be directly identified with the dynamical exponent $z\left(\delta_{\mathrm{IAF}}\right)$ of this phase. This picture thus predicts that the typical separation of such regions is of order $\omega^{-1 / z}$, while their lengths are only of order $|\ln \omega|$, in complete agreement with the schematic RG approach.

\section{Singlet $R G$ description of the random dimer phases: A review}

While the effects of dimerization are not understood in detail in all regimes, it is possible ${ }^{9}$ to use the singlet RG and follow the flows for a chain with full Heisenberg symmetry and for a chain in the vicinity of the $X X$-RS point. In these cases, a mapping to the off-critical flows of the RTFIM provides a detailed characterization of the so-called RD phases that result. In either case, the picture that emerges can be summarized as follows: ${ }^{9}$ For concreteness, assume $\delta>0$. If disorder is strong and $\delta \ll 1$, then the even and the odd bonds renormalize essentially as in the corresponding RS state till the log-energy scale $\Gamma \sim \Gamma_{\delta} \equiv 1 / \delta$. Beyond this scale, the remaining odd bonds rapidly become much weaker relative to the remaining even bonds; the distribution of the even logcouplings $P^{\mathrm{e}}(\zeta \mid \Gamma)=\int d l P^{\mathrm{e}}(\zeta, l \mid \Gamma)$ approaches some limiting distribution with a finite but large width, while distribution of the odd log-couplings $P^{\mathrm{o}}(\zeta \mid \Gamma)=\int d l P^{\mathrm{o}}(\zeta, l \mid \Gamma)$ grows infinitely broad. In the RG language, the system renormalizes to some point on a line of RD fixed points (from this point of view, the RS states at $\delta=0$ represent critical points separating RD fixed points with opposite dimerization, see Fig. 2). The corresponding joint distributions of the log-couplings and the lengths have been worked out in Ref. 6; here we only note that $P^{\mathrm{e}}(\zeta \mid \Gamma)=\tau_{0}(\Gamma) e^{-\zeta \tau_{0}(\Gamma)}$ with $\tau_{0}(\Gamma) \approx 2 \delta$, while $P^{\mathrm{o}}(\zeta \mid \Gamma)=u_{0}(\Gamma) e^{-\zeta u_{0}(\Gamma)} \quad$ with $\quad u_{0}(\Gamma) \approx 2 \delta e^{-2 \delta \Gamma}$. The ground state again consists of singlet pairs made up of one spin on an even site $i$ and a second spin on some odd site $j$. Note, however, that while $i>j$ and $i<j$ are equally probable in the RS state, in the RD phase with $\delta>0$ one almost always has $j>i$ (with the exception of a few high-energy pairs of small spatial extent).

\section{B. Dynamic structure factor}

In this section, we summarize our calculations of the dynamic structure factor in the different regions of the phase diagram of spin-1/2 XXZ chains. Our approach has already been reviewed in general terms in Sec. II D, and our calculations here represent one of the simplest examples of this approach at work.

We begin by considering only the leading term in the perturbative expansion for the renormalized spin operators; the results obtained in this manner give the correct leading behavior at low frequencies (some justification of this is given in Sec. III E, where we discuss the role of higher-order terms).

\section{Random singlet states}

The leading-order "operator renormalizations" needed are particularly simple: the spin operator $\vec{s}$ remains unchanged for each of the "surviving" spins and is effectively zero for each of the "decimated" spins (i.e., spins that are already locked into singlets with other spins).

Consider first $S^{z z}(k, \omega)$; in our formulation of the singlet RG, Sec. III A, the following analysis applies to a general $X X Z$ singlet state (i.e., remains valid so long as the ground state does not have IAF order). Consider two spins $L$ and $R$ connected by a strong bond $\left(\widetilde{J}^{\perp}, \widetilde{J}^{z}\right)$ in the renormalized theory with cutoff $\Omega_{\text {final }}$. The spin operators $s_{L / R}^{z}$ connect the singlet ground state of this pair only to the triplet state $\left|t_{0}\right\rangle$ (with $m_{z}=0$ ), which is separated from the singlet state by a gap $\widetilde{J}^{\perp}$. Therefore, the energy-scale $\Omega_{\text {final }}$ at which we stop the RG is $\Omega_{\text {final }}=\omega$ in this case (remember that the cutoff was defined as $\Omega=\max \left\{J^{\perp}\right\}$ ). We thus consider the renormalized spectral sum

$$
S^{z z}(k, \omega)=\frac{1}{L} \tilde{\sum_{m}}\left|\left\langle m\left|\sum_{j} e^{i k x_{j}} \widetilde{S}_{j}^{z}\right| 0\right\rangle\right|^{2} \delta\left(\omega-\widetilde{E}_{m}\right),
$$

where the tildes remind us of the fact that this spectral sum now refers to the new Hamiltonian with energy cutoff $\Omega_{\text {final }}=\omega$; this renormalized Hamiltonian has $n_{\Gamma_{\omega}}$ spins per unit length with the distribution of couplings and bond lengths characteristic of the fixed point to which the system flows in the low-energy limit. The sum in Eq. (18) is dominated by the excitations to the triplet state $\left|t_{0}\right\rangle$ of pairs of spins connected by the (renormalized) bonds with $\widetilde{J}^{\perp}=\omega$; these pairs are precisely the ones that are being eliminated at this energy scale. The corresponding matrix element for each such pair is simply $\left(1-e^{i k \tilde{l}}\right) / 2$, where $\tilde{l}$ is the length of the bond connecting the pair; this allows us to write

$$
S^{z z}(k, \omega) \sim n\left(\Gamma_{\omega}\right) \int d l d \zeta\left|1-e^{i k l}\right|^{2} P\left(\zeta, l \mid \Gamma_{\omega}\right) \delta\left(\omega-\omega e^{-\zeta}\right)
$$

for $\omega \ll \Omega_{0}$ in any RS state.

The calculation of $S^{+-}(k, \omega)$ is more involved since the gap to the relevant triplet excited state $\left|t_{1}\right\rangle$ (with $m_{z}=1$ ) of a pair of spins connected by a strong bond $\left(\widetilde{J}^{\perp}, \widetilde{J}^{z}\right)$ is now $\left(\widetilde{J}^{\perp}+\widetilde{J}^{z}\right) / 2$. We consider each of the three cases $(X X, X X X$, and $X X Z C$ ) separately: (1) In the $X X X$ case, the Heisenberg symmetry of the problem guarantees that $S^{x x}=S^{y y}=S^{z z}$. (2) 
When the system approaches the $X X$ point at low energies, we have $\widetilde{J}^{z} \ll \widetilde{J}^{\perp}$ implying that the relevant gap is approximately $\widetilde{J}^{\perp} / 2$. Thus, to calculate $S^{+-}(k, \omega)$ we now have to stop the RG at the scale $\Omega_{\text {final }}=2 \omega$. From Eq. (19), it is clear that this leaves our answer unchanged except for the values of various non-universal scale factors. (3) The $X X Z C$ critical point needs special attention. In this case $\widetilde{J}^{z} / \widetilde{J}^{\perp}$ can have a range of values. As a result, the excited states that dominate the spectral sum Eq. (4) are not simply obtained by stopping the RG at any particular $\Omega_{\text {final }}$ and looking at the singlets forming only at this scale. Instead, for any $\Omega_{\text {final }} \in(0,2 \omega)$ there will be some singlets formed at this scale that will contribute to the spectral sum, namely, the pairs coupled by strong bonds with $\widetilde{J}^{\perp}=\Omega_{\text {final }}$ and $\widetilde{J}^{z}=2 \omega-\Omega_{\text {final }}$. Note that there is no double-counting here since we are considering only the pairs that are being eliminated at each energy scale. Thus, we have

$$
\begin{aligned}
S^{+-}(k, \omega) \sim & \int d \Gamma \operatorname{dld} \operatorname{Dn}(\Gamma)\left|1-e^{i k l}\right|^{2} P(0, D, l \mid \Gamma) \\
& \times \delta\left(\omega-\Omega_{0} e^{-\Gamma}\left[1+e^{D}\right] / 2\right) .
\end{aligned}
$$

Rewriting this in terms of the scaling probability distribution $\mathcal{P}_{2}$ and using the delta function to do the $\Gamma$ integral gives us

$$
\begin{aligned}
S^{+-}(k, \omega) \sim & \frac{1}{\omega \Gamma_{\omega}} \int d \bar{l} d \bar{D} \frac{n\left[\Gamma_{\omega} \Upsilon_{\omega}(\bar{D})\right]}{\Upsilon_{\omega}^{3+\psi}(\bar{D})}\left|1-e^{i \bar{k} \bar{l}}\right|^{2} \\
& \times \mathcal{P}_{2}\left(0, \frac{\bar{D}}{\Upsilon_{\omega}^{\psi}(\bar{D})}, \frac{\bar{l}}{\Upsilon_{\omega}^{2}(\bar{D})}\right),
\end{aligned}
$$

where we have defined $\bar{D}=D / \Gamma_{\omega}^{\psi}, \bar{l}=l / \Gamma_{\omega}^{2}, \bar{k}=k \Gamma_{\omega}^{2}$, and

$$
\Upsilon_{\omega}(\bar{D})=1+\frac{\ln \left(1+e^{\bar{D} \Gamma_{\omega}^{\psi}}\right)-\ln 2}{\Gamma_{\omega}}
$$

Now, since $\psi<1$, it is permissible to take the $\Gamma_{\omega} \rightarrow \infty$ limit of $\Upsilon_{\omega}(\bar{D})$ before doing the $\bar{D}$ integral, in other words, we can replace $Y$ by 1 in the low-energy limit. The $\bar{D}$ integral can then be done trivially, and the final expression is identical in form to Eq. (19). More physically, a given bond $\left(J^{\perp}, J^{z}\right)$ is described fairly well (on a logarithmic scale) by one of these two couplings; we chose the characteristic scale to be $J^{\perp}$. Now, the random anisotropy leads to an uncertainty $\left|\ln \left(J^{\prime} / J^{\perp}\right)\right| \sim \Gamma^{\psi}$ in the corresponding log-energy scale. This uncertainty is much smaller than the already existing typical spread in the log-energies or the typical log-energies themselves, which are both of order $\Gamma$. The leading behavior at low frequencies is therefore not affected.

Thus, in the limit of low frequencies both $S^{z z}(k, \omega)$ and $S^{+-}(k, \omega)$ can be expressed in terms of the scaling probability distribution $\mathcal{P}_{1}$ as

$$
S(k, \omega) \sim \frac{n\left(\Gamma_{\omega}\right)}{\omega \Gamma_{\omega}} \int d \bar{l}\left|1-e^{i \bar{k} \bar{l}}\right|^{2} \mathcal{P}_{1}(0, \bar{l}) .
$$

Let us first focus on the regime $|q| \equiv|k-\pi / a| \ll a^{-1}$. Note that all unscaled lengths $l$ are odd multiples of the unit length $a$, and therefore $e^{i k l}=-e^{i q l}$. The integral in Eq. (23) can be evaluated using the characterization of the function $\mathcal{P}_{1}(0, y)$ available in Ref. 6; the result is the following rather unusual scaling form at all three RS fixed points:

$$
S\left(k=\frac{\pi}{a}+q, \omega\right)=\frac{\mathcal{A}}{l_{v} \omega \ln ^{3}\left(\Omega_{0} / \omega\right)} \Phi\left[\left|q l_{v}\right|^{1 / 2} \ln \left(\Omega_{0} / \omega\right)\right] .
$$

Note that we have suppressed the component labels on $S(k, \omega)$ as the two independent components obey the same scaling form, but with different values in general of the numerical constant $\mathcal{A}$ and the microscopic length-scale $l_{v}$. The universal function $\Phi(x)$ can be written as

$$
\Phi(x)=1+x \frac{\cos (x) \sinh (x)+\sin (x) \cosh (x)}{\cos ^{2}(x) \sinh ^{2}(x)+\sin ^{2}(x) \cosh ^{2}(x)} .
$$

The resulting $S(k, \omega)$ is shown on Fig. 3. There is a fairly straightforward interpretation of the main features of this line shape: The peak at $q=0$ (i.e., at $k=\pi / a$ ) reflects the predominantly antiferromagnetic character of the low-energy fluctuations; in our language, this is a direct consequence of the fact that the (renormalized) bonds all have odd lengths in units of $a$. The strongly damped oscillations with the period and the decay scale both of order $\Gamma_{\omega}^{-2}$ express the properties of the distribution of lengths of the strong bonds: both the average and the RMS fluctuation of this distribution of lengths are of order $\Gamma_{\omega}^{2}$.

While this result is interesting, one needs to analyze the effects of higher-order terms in the operator renormalizations before accepting its consequences for possible neutron scattering experiments. We will argue in Sec. III E that higherorder corrections do not modify the functional form (25) of the features in $S(k, \omega)$ at fixed $\omega$ but only add an "incoherent" background (of strength comparable to that of the features) and suppress the amplitude of the features by a nonuniversal multiplicative factor of order one.

A similar scaling function can be derived for the regime $|k| \ll a^{-1}$. Repeating the above analysis gives

$$
S(k, \omega)=\frac{\mathcal{A}^{\prime}}{l_{v} \omega \ln ^{3}\left(\Omega_{0} / \omega\right)} \widetilde{\Phi}\left[\left|k l_{v}\right|^{1 / 2} \ln \left(\Omega_{0} / \omega\right)\right],
$$

with $\widetilde{\Phi}(x)=2-\Phi(x)$, and $\mathcal{A}^{\prime}$ an order-one numerical constant. This scaling function vanishes for $k \rightarrow 0$; for small $k$ we have $S(k, \omega) \sim l_{v} k^{2} \ln \left(\Omega_{0} / \omega\right) / \omega$. We must therefore consider the possibility that higher-order corrections may overwhelm this scaling result and render it irrelevant. This is indeed expected to happen for $S^{+-}(k, \omega)$ away from the $X X X$ point. However, we expect the scaling result to be valid quite generally for $S^{z z}(k, \omega)$ because spin conservation guarantees that the higher-order corrections to $S^{z z}(k, \omega)$ must also vanish as $k \rightarrow 0$ (see Sec. III E for a detailed discussion of this point). 


\section{Dynamic structure factor in random dimer phases: "Sharpness" of the Griffiths regions}

Next, we consider the spin dynamic structure factor in the $X X$ and $X X X$ random dimer phases introduced in Sec. III A 3. The same approach as for the RS states goes over unchanged, and we write

$$
\begin{aligned}
S(k, \omega) \sim & n\left(\Gamma_{\omega}\right) \int d l d \zeta\left|1-e^{i k l}\right|^{2} \\
& \times\left[P^{0}\left(\zeta, l \mid \Gamma_{\omega}\right)+P^{\mathrm{e}}\left(\zeta, l \mid \Gamma_{\omega}\right)\right] \\
& \times \delta\left(\omega-\omega e^{-\zeta}\right)
\end{aligned}
$$

for both $S^{z z}(k, \omega)$ and $S^{+-}(k, \omega)$ in both the $X X$ and $X X X$ RD phases. (We are again being sloppy about the distinction between $\Gamma_{\omega}$ and $\Gamma_{\omega / 2}$, as this can be absorbed in the definition of the nonuniversal scale factors that enter our expressions.)

Using the results of Ref. 6, it is a simple matter to obtain the full crossover from the RS-like behavior of the structure factor in the regime $1 \ll \Gamma_{\omega} \leq \Gamma_{\delta}$ to the behavior characteristic of the RD phase in the regime $\Gamma_{\omega} \gg \Gamma_{\delta}$. Here, we focus on the behavior in the regime $\Gamma_{\omega} \gg \Gamma_{\delta}$, as this exhibits some rather unusual features. At these low energies, the even bonds dominate over the odd bonds, and the contribution of the odd bonds to the sum Eq. (27) is negligible (we are assuming $\delta>0$ for concreteness). For wave vectors in the vicinity of $k=\pi / a$ with $|q| \equiv|k-\pi / a| \ll \delta^{2} / l_{v}$ (i.e., probing lengths larger than the correlation length $\xi_{\mathrm{av}} \sim l_{v} / \delta^{2}$ ) we obtain

$$
S(k, \omega)=\frac{\mathcal{C}|\delta|^{3} \Omega_{0}^{-1 / z_{\mathrm{RD}}}}{l_{v} \omega^{1-1 / z_{\mathrm{RD}}}}\left[1+\cos \left(l_{v} q \Gamma_{\omega} /|\delta|\right) e^{-c l_{v}^{2} q^{2} \Gamma_{\omega} /|\delta|^{3}}\right]
$$

where $\mathcal{C}$ and $c$ are some order-one constants, and we have chosen to write the power-law prefactor in terms of the dynamical exponent $z_{\mathrm{RD}}$. (As far as our RG calculations are concerned, $z_{\mathrm{RD}}^{-1}=2|\delta|$ for small $|\delta|$. However, the effective value of $\delta$ that enters this expression is expected to acquire a nonuniversal multiplicative renormalization from the highenergy physics, and the only reliable statement we can make is that $z_{\mathrm{RD}}^{-1} \sim|\delta|$ for small enough $|\delta|$.) This result has a striking oscillatory structure (see Fig. 4) that is not suppressed significantly by the exponential factor, since $\sqrt{\Gamma_{\omega} /|\delta|^{3}}$ $\ll \Gamma_{\omega} /|\delta|$ in the regime under consideration. This is best understood as a novel signature of the sharply defined geometry of the rare Griffiths regions that contribute to the scattering at a given low energy (i.e., that are filtered out by their energy). More precisely, the average length of such regions is of order $l_{v} \Gamma_{\delta}^{2}\left(\Gamma_{\omega} / \Gamma_{\delta}\right)=l_{v} \Gamma_{\omega} /|\delta|$, while the root-meansquare fluctuations in the length are only of order $l_{v} \Gamma_{\delta}^{2} \sqrt{\Gamma_{\omega} / \Gamma_{\delta}}=l_{v} \sqrt{\Gamma_{\omega} /|\delta|^{3}}$. Our results thus suggest that low-energy INS experiments would be able to pick up the sharply defined geometry of such Griffiths regions in the RD phases in one dimension.

This feature of the Griffiths regions in one dimension was noted in Ref. 6, Sec. IVB, in the context of the RTFIM, where it was conjectured also that other properties of such low-energy regions are likewise sharply defined: for example, in the disordered phase of the RTFIM, the magnetic moment of the Griffiths regions with a given characteristic energy is sharply defined ${ }^{21}$ and proportional to the (sharply defined) length of such regions. (In fact, similar "sharpness", is expected to hold for any bond "property" that "rides" on top of the singlet $\mathrm{RG}$ via recursion relation $\tilde{x}=x_{1}+x_{3}$ $+\Upsilon x_{2}$ when bond $J_{2}$ is eliminated.) We expect to see a signature of this sharpness of the Griffiths regions also in the dynamic structure factor $S^{z z}$ in the IAF Griffiths phase (see below) and also in the Griffiths phases of the onedimensional RTFIM (Sec. V C). Finally, an interesting question, which we leave unanswered for now, is whether similar sharpness in the properties of the Griffiths regions at a given energy occurs and has observable consequences in higher dimensions as well, e.g., in the disordered phase of the $d>1$ RTFIM.

\section{IAF Griffiths phase}

Let us first consider $S^{z z}(k, \omega)$ in the IAF Griffiths phase. As discussed in Sec. III A 2, the dominant low-energy excitations in this phase are classical domain walls. However, it is clear that such excitations do not contribute at all to $S^{z z}(k, \omega)$, since they cannot be excited from the ground state by the action of operators like $\hat{s}_{k}^{z}$, which conserve the total $s_{\text {tot }}^{z}$. The leading excitations that do contribute to $S^{z z}$ can clearly be identified in the RG picture with the $m_{z}=0$ excited states of pairs of superspins, with each pair connected by a bond with $\widetilde{J}^{\perp} \sim \omega$ and forming a singlet (note that this is true regardless of the value of the corresponding $\widetilde{J}^{z}$ ). Now, it is easy to generate a weak $\widetilde{J}^{\perp}$ coupling of order $\omega$ in the IAF phase, since any typical region of length $L$ will have an effective $\widetilde{J}^{\perp}$ of order $\Omega_{0} e^{-c_{x} L}$ (and an effective $\widetilde{J}^{z}$ typically much stronger). What is more difficult is to isolate such a region from becoming a part of a larger cluster, otherwise this region cannot support spin fluctuations at frequency $\omega$. For this, we need two rare RS-like segments (domain walls) with $\widetilde{J}^{z} \lesssim \omega$, one on each side of our (typical) region. Thus, we need two domain walls, which are usually separated by a large distance of order $\omega^{-1 / z}$, to occur close to each other; the "density" of such occurrences is $\sim \omega^{2 / z_{\mathrm{IAF}}}$. The separation of the two domain walls - the length of the IAF-ordered cluster that they isolate-must be of order $|\ln \omega|$. More precisely, if the IAF-ordered cluster has length $L$, it can be thought of as consisting of the $n \sim L / \Gamma_{\delta_{\text {IAF }}}^{2}$ strongly Isingcoupled spins that are active at the crossover scale; the effective bonds connecting these spins at the crossover scale typically satisfy $\ln \left(\widetilde{J}^{\perp} / \widetilde{J}^{z}\right) \sim-\Gamma_{\delta_{\text {IAF }}}$. The requirement that the spin-flip coupling for this cluster is $\omega$ fixes the length of this cluster to be $L=l_{v} \Gamma_{\omega} \Gamma_{\delta_{\mathrm{IAF}}}$, while the uncertainty in this length can only be of order $l_{v} \sqrt{\Gamma_{\omega} \Gamma_{\delta_{\mathrm{IAF}}}^{3}} \ll l_{v} \Gamma_{\omega} \Gamma_{\delta_{\mathrm{IAF}}}$.

We are now ready to calculate $S^{z z}(k=\pi / a+q, \omega)$ in the regime $|q|^{-1} \gg l_{v} \Gamma_{\delta_{\mathrm{IAF}}}^{2}$, in addition to $\omega \ll \Omega_{\delta_{\mathrm{IAF}}}$. The leading- 
order renormalization of the $s_{j}^{z}$ in the cluster RG is simple: $s_{j}^{z}$ is renormalized to $(-1)^{j} s_{c}^{z}$ for each spin $j$ that is active in some cluster $c$, and renormalizes to zero for every spin that forms a singlet. Assuming that such clusters "look" fairly uniform on the length scales larger than $l_{v} \Gamma_{\delta_{\mathrm{IAF}}}^{2}$, and adding up the contributions from all such isolated clusters with effective spin fluctuation frequency $\omega$, we obtain

$$
\begin{aligned}
S^{z z}\left(k=\frac{\pi}{a}+q, \omega\right)= & \frac{\mathcal{C}^{\prime}\left|\delta_{\mathrm{IAF}}\right|^{7 \theta} \Omega_{0}^{-2 / z} \mathrm{IAF}}{q^{2} l_{v}^{3} \omega^{1-2 / z_{\mathrm{IAF}}}} \\
& \times\left[1-\cos \left(q l_{v} \Gamma_{\omega} /\left|\delta_{\mathrm{IAF}}\right|^{\theta}\right)\right. \\
& \times e^{\left.-c q^{2} l_{v}^{2} \Gamma_{\omega} /\left|\delta_{\mathrm{IAF}}\right|^{3 \theta}\right],}
\end{aligned}
$$

where $\mathcal{C}^{\prime}$ and $c$ are some order-one constants and the power of the $\delta_{\mathrm{IAF}}$ that appears in the prefactor has been fixed by demanding consistency with the off-critical scaling form

$$
S^{z z}\left(k=\frac{\pi}{a}+q, \omega\right)=\frac{\mathcal{A}}{l_{v} \omega \Gamma_{\omega}^{3}} \Psi\left(\frac{\Gamma_{\omega}}{\Gamma_{\delta_{\mathrm{IAF}}}},\left|q l_{v}\right|^{1 / 2} \Gamma_{\omega}\right),
$$

with $\Psi(0, y)=\Phi(y)$. Note also that the overall $1 / q^{2}$ dependence is a consequence of the fact that the spins contributing to the scattering have been taken to be distributed uniformly over a sharply defined region (the cluster); we expect this to cross over to a much faster decay at large momenta (such that $|q|^{-1} \sim l_{v} \Gamma_{\delta_{\text {IAF }}}^{2}$ ) well outside the range of validity of our scaling picture.

The situation is quite different for $S^{+-}(k, \omega)$. As we shall see in Sec. III C, the renormalization of the $s_{j}^{ \pm}$spin operators is quite nontrivial, and we are unable to make an equally detailed prediction for $S^{+-}$. However, we expect that the matrix element for producing domain-wall excitations with energies of order $\omega$ by the action of $s^{ \pm}$on the ground state is strongly suppressed as some power of $\omega$, giving rise to a correspondingly small value for $S^{+-}(k, \omega)$ at small $\omega$.

\section{Average local autocorrelations}

The same approach can be used to calculate average autocorrelation functions, and this section is devoted to a brief account of our results.

We consider the local dynamical susceptibilities

$$
\chi_{j j}^{\alpha \alpha}(\omega)=\sum_{m}\left|\left\langle m\left|s_{j}^{\alpha}\right| 0\right\rangle\right|^{2} \delta\left(\omega-E_{m}\right),
$$

where $\alpha=z$ or $\alpha=x$. A knowledge of the low-frequency behavior of these susceptibilities can immediately be translated into information about the long-time limit of the corresponding imaginary-time autocorrelation functions

$$
C_{j j}^{\alpha \alpha}(\tau)=\left\langle s_{j}^{\alpha}(\tau) s_{j}^{\alpha}(0)\right\rangle .
$$

\section{1. $R S$ states and $R D$ phases}

As long as one is interested only in averages of such local quantities (over different realizations of disorder), it again suffices to consider only the leading-order spin-operator renormalizations. We thus already have all the ingredients needed to calculate these average dynamical susceptibilities: our basic approach is familiar enough by now, and the relevant results of Ref. 4 for the renormalized bond distributions have already been reviewed in Sec. III A. Below, we will be correspondingly brief. We first give our results for the average local dynamical susceptibilities and then translate these to results for the long-time behavior of the corresponding average autocorrelation functions. ${ }^{22}$ The leading behavior is the same for both $\alpha=z$ and $\alpha=x$, so we drop all superscripts.

For a bulk-spin, we obtain

$$
\left[\chi_{\mathrm{loc}}\right]_{\mathrm{av}}(\omega) \sim \frac{n\left(\Gamma_{\omega}\right)}{\omega}\left[P_{0}^{\mathrm{e}}\left(\Gamma_{\omega}\right)+P_{0}^{\mathrm{o}}\left(\Gamma_{\omega}\right)\right] .
$$

For the critical RS states $\left(P^{\mathrm{e}}=P^{\mathrm{o}}\right)$ we find

$$
\begin{gathered}
{\left[\chi_{\mathrm{loc}}\right]_{\mathrm{av}}(\omega) \sim \frac{1}{\omega|\ln \omega|^{3}},} \\
{\left[C_{\mathrm{loc}}\right]_{\mathrm{av}}(\tau) \sim \frac{1}{|\ln \tau|^{2}},}
\end{gathered}
$$

while off-critical—in the RD phases—we find

$$
\begin{gathered}
{\left[\chi_{\mathrm{loc}}\right]_{\mathrm{av}}(\omega) \sim \frac{|\delta|^{3}}{\omega^{1-1 / z_{\mathrm{RD}}}},} \\
{\left[C_{\mathrm{loc}}\right]_{\mathrm{av}}(\tau) \sim \frac{|\delta|^{3}}{\tau^{1 / z_{\mathrm{RD}}}} .}
\end{gathered}
$$

Similarly, for an end-spin $s_{1}$ of a semi-infinite chain (with $j \geqslant 1$ ) we obtain

$$
\left[\chi_{1}\right]_{\mathrm{av}}(\omega) \sim \frac{P_{0}^{\mathrm{e}}\left(\Gamma_{\omega}\right) P_{0}^{\mathrm{o}}\left(\Gamma_{\omega}\right)}{\omega} .
$$

For the RS states we find

$$
\begin{gathered}
{\left[\chi_{1}\right]_{\mathrm{av}}(\omega) \sim \frac{1}{\omega|\ln \omega|^{2}},} \\
{\left[C_{1}\right]_{\mathrm{av}}(\tau) \sim \frac{1}{|\ln \tau|},}
\end{gathered}
$$

and in the RD phases

$$
\begin{gathered}
{\left[\chi_{1}\right]_{\mathrm{av}}(\omega) \sim \frac{\delta^{2}}{\omega^{1-1 / z_{\mathrm{RD}}}},} \\
{\left[C_{1}\right]_{\mathrm{av}}(\tau) \sim \frac{\delta^{2}}{\tau^{1 / z_{\mathrm{RD}}}} .}
\end{gathered}
$$

\section{IAF Griffiths phase}

In the IAF phase, unlike in the singlet states, we need to make a distinction between $\chi^{z z}$ and $\chi^{x x}$. Consider first 
$\left[\chi_{\mathrm{loc}}^{z z}\right]_{\mathrm{av}}(\omega)$. From our previous discussion of the IAF phase, it is clear that, in the regime $\omega \ll \Omega_{\delta_{\text {IAF }}}$, the dominant contributions come from IAF-ordered clusters of lengths $\sim \Gamma_{\omega} \Gamma_{\delta_{\text {IAF }}}$ (i.e., with effective spin-flip couplings of order $\omega$ ) that are isolated from the rest of the system by domain walls with $\widetilde{J}^{z} \lesssim \omega$ on either side. From the scaling picture of the phase, we get

$$
\begin{gathered}
{\left[\chi_{\mathrm{loc}}^{z z}\right]_{\mathrm{av}}(\omega) \sim \delta_{\mathrm{IAF}}^{4 \theta} \frac{\Omega_{0}^{-2 / z_{\mathrm{IAF}}} \ln \left(\Omega_{0} / \omega\right)}{\omega^{1-2 / z_{\mathrm{IAF}}}},} \\
{\left[C_{\mathrm{loc}}^{z z}\right]_{\mathrm{av}}(\tau) \sim \delta_{\mathrm{IAF}}^{4 \theta} \frac{\ln \left(\Omega_{0} \tau\right)}{\left(\Omega_{0} \tau\right)^{2 / z_{\mathrm{IAF}}}} .}
\end{gathered}
$$

The analysis is more complicated for $\left[\chi_{\mathrm{loc}}^{x x}\right]_{\mathrm{av}}(\omega)$, and we can only make a plausible estimate for this quantity. This is because the $x$ and $y$ components of the spin operators renormalize in a nontrivial way under the cluster RG. The origin of this difficulty may be seen as follows: Consider, for example, combining three spins $s_{2}, s_{3}$, and $s_{4}$, connected by strong $J_{2}^{z}$ and $J_{3}^{z}$, into a superspin $\tilde{s}_{(234)}$. To zeroth order, all three operators $s_{2}^{+}, s_{3}^{+}$, and $s_{4}^{+}$renormalize to zero. To first order, $s_{2}^{+}$and $s_{4}^{+}$renormalize to $\left(s_{2}^{+}\right)^{\mathrm{eff}}=-s_{1}^{+} J_{1}^{\perp} / J_{2}^{z}$ $-\tilde{s}_{(234)}^{+} 2 J_{3}^{\perp} / J_{2}^{z}, \quad\left(s_{4}^{+}\right)^{\mathrm{eff}}=-s_{5}^{+} J_{4}^{\perp} / J_{3}^{z}-\tilde{s}_{(234)}^{+} 2 J_{2}^{\perp} / J_{4}^{z}$, while $s_{3}^{+}$renormalizes to zero to this order. Roughly speaking, the original spin-flip operators of the (active) spins have projections onto the remaining effective cluster spin-flip operators with components given by the ratio of the corresponding effective spin-flip couplings to the original spin-flip couplings.

Now, the dominant contributions to $\left[\chi_{\text {loc }}^{x x}\right]_{\mathrm{av}}(\omega)$ come from the low-energy (of order $\omega$ ) domain-wall excitations, which are represented in the RG picture by the bonds with $\widetilde{J}^{z} \sim \omega$ connecting the effective spins (clusters) in the effective theory with the renormalized cutoff $\omega$. The matrix element for producing such an excitation by a bare spin-flip operator of a spin active in one of these clusters will be of order of the corresponding $\widetilde{J}^{\perp}$, while the number of such spins contributing will be of order of some effective "moment" $\mu_{x}$ of this cluster. Because of the matrix element proportional to $\widetilde{J}^{\perp}$, there will be a significant contribution only if this $\widetilde{J}^{\perp}$ is also of order $\omega$. As we have already seen, this can happen only if such an IAF-ordered cluster has length of order $\Gamma_{\omega}$ and is isolated from the rest by RS-like regions (domain walls) with $J^{z} \leq \omega$ on either side. We already know how to estimate the number density of such Griffiths regions. As far as the effective moment $\mu_{x}$ of such an IAF cluster is concerned, we can only make a crude estimate that bounds it from above by the number of spins that are active in this cluster: $\mu_{x}(\omega) \leq \Gamma_{\omega}$; however, we are unable to obtain the precise power of the logarithm that enters the energy dependence of the effective moment. We therefore leave out the logarithmic correction, and only write the dominant power-law part of our estimate:

$$
\begin{gathered}
{\left[\chi_{\mathrm{loc}}^{x x}\right]_{\mathrm{av}}(\omega) \sim \omega^{1+2 / z \mathrm{IAF}},} \\
{\left[C_{\mathrm{loc}}^{x x}\right]_{\mathrm{av}}(\tau) \sim \frac{1}{\tau^{2+2 / z}} .}
\end{gathered}
$$

\section{Spin transport}

This section is devoted to a discussion of the dynamical spin conductivity $\sigma^{\prime}(\omega)$ in the spin-1/2 $X X Z$ chains. Our task here is to evaluate the Kubo formula Eq. (5) in the low-frequency limit. For the RS and RD states, we will use information available from the scaling solutions to the singlet $R G$ recursion relations to achieve this, while in the IAF phase, we will use the scaling picture of the Griffiths phase we have developed earlier. Our results for the dynamical conductivity are summarized in Figs. 1 and 2.

\section{Random singlet states}

We first need to work out the rules that govern the renormalizations of the current operators. Assume once again that $J_{23}^{\perp}$ is the strongest bond. We wish to work out perturbatively the renormalized operators $\tilde{\tau}_{1 / 2 / 3}$ that we trade in $\tau_{1 / 2 / 3}$ for, when we freeze spins 2 and 3 in their singlet ground state (the other current operators to the left and right of this segment are left unchanged to leading order by the renormalization). Now, note that these other operators have overall scale factors in them that are nothing but the corresponding $J^{\perp}$ couplings. In order to be consistent, we clearly need to work out $\tilde{\tau}_{1 / 2 / 3}$ correct to $O\left(\widetilde{J}_{14}^{\perp}\right)$ (where $\widetilde{J}_{14}$ is the effective bond connecting spins 1 and 4 after we freeze out spins 2 and 3) by adding the effects of virtual fluctuations to the projections of $\tau_{1 / 2 / 3}$ into the singlet subspace. An explicit calculation gives the simple result that all three operators renormalize to the same operator $\tilde{\tau}_{1 / 3}=\tilde{\tau}_{2}=i \widetilde{J}_{14}^{\perp}\left(s_{1}^{+} s_{4}^{-}-s_{4}^{+} s_{1}^{-}\right) / 2$, which we will denote henceforth by $\tilde{\tau}_{1}$ for consistency of notation.

As we carry out the RG, the above result implies that the total current operator $\sum_{j=1}^{L} \tau_{j}$ entering Eq. (5) renormalizes to $\widetilde{\Sigma}_{j} \widetilde{l}_{j} \tilde{\tau}_{j}$, where $j$ now labels the remaining sites of the renormalized system, and the $\widetilde{l}_{j}$ are the lengths of the corresponding renormalized bonds. (Note that this result makes sense physically and is a consequence of spin conservation: when a magnetic field with a uniform gradient is applied along the length of the chain, the effective lengths $\widetilde{l}_{j}$ measure the "phase" along the chain of this "driving potential.") Consider two spins connected by a strong bond $\left(\widetilde{J}^{\perp}, \widetilde{J}^{z}\right)$ in the renormalized theory with cutoff $\Omega_{\text {final }}$. Since the current operator living on this bond connects the singlet ground state of the pair only to the triplet state $\left|t_{0}\right\rangle$ separated from the singlet by a gap $\widetilde{J}^{\perp}$, we choose $\Omega_{\text {final }}=\omega$ and consider the renormalized spectral sum

$$
\sigma^{\prime}(\omega)=\frac{1}{\omega L} \tilde{\sum}_{m}^{\sim}\left|\left\langle m\left|\tilde{\sum}_{j}^{\sim} \tilde{l}_{j} \tilde{\tau}_{j}\right| 0\right\rangle\right|^{2} \delta\left(\omega-\widetilde{E}_{m}\right) .
$$

This spectral sum is dominated by precisely the $\left|t_{0}\right\rangle$ triplet excitations of pairs of spins that are connected by the (effec- 
tive) bonds with $\widetilde{J}^{\perp}=\omega$ and are being eliminated at this energy scale; the corresponding matrix element is just $\tilde{l} \omega / 2$, where $\tilde{l}$ is the length of the bond connecting the pair. In the thermodynamic limit, we thus have

$$
\sigma^{\prime}(\omega) \sim \frac{n\left(\Gamma_{\omega}\right)}{\omega} \int d l d \zeta \omega^{2} l^{2} P\left(\zeta, l \mid \Gamma_{\omega}\right) \delta\left(\omega-\omega e^{-\zeta}\right) .
$$

This immediately yields our central result

$$
\sigma^{\prime}(\omega)=\mathcal{K}_{\mathrm{RS}} l_{v} \ln \left(\Omega_{0} / \omega\right),
$$

valid for $\omega \ll \Omega_{0}$. Here, $\mathcal{K}_{\mathrm{RS}}$ is an order-one numerical constant, $l_{v}$ is the microscopic length-scale defined earlier, and $\Omega_{0}$ is the microscopic energy cutoff. Notice that this analysis holds equally well at all three RS fixed points, which differ only in the corresponding values of the nonuniversal scale factors.

A brief digression is in order, before we go on to discuss this result: The real part of the dynamical conductivity can be related (on general grounds) to the behavior of the dynamic-structure factor $S^{z z}(k, \omega)$ near $k=0$

$$
\sigma^{\prime}(\omega)=\omega \frac{1}{2} \frac{d^{2}}{d k^{2}} S^{z z}(k, \omega) ;
$$

this can be checked by comparing directly the corresponding spectral sums and noticing that the action of the two operators $\mathcal{T}=\Sigma_{j} \tau_{j}$ and $\mathcal{V}=\Sigma_{j} j \sigma_{j}^{z}$ on the eigenstates of the Hamiltonian $\mathcal{H}$ are related through $\mathcal{T}=i[\mathcal{H}, \mathcal{V}]$. It is easy to check, using the scaling form Eq. (26), that our result for the conductivity is consistent, as it must be, with our previously derived result for the dynamic structure factor.

Going back to Eq. (42), we see that $\sigma^{\prime}(\omega)$ diverges logarithmically for small $\omega$ in the unusual "spin-metal" phase controlled by the $X X$ fixed point as well as at the critical points ( $X X X$ and $X X Z C)$ separating this phase from the "insulating" phase with Ising antiferromagnetic order in the ground state. Note that this "metal-insulator" transition has the curious feature that the quantum critical points separating the conducting phase from the insulating phase have the same $T=0$ transport properties as the conducting phase.

\section{IAF Griffiths phase}

On the insulating side, we expect $\sigma^{\prime}(\omega)$ to be suppressed

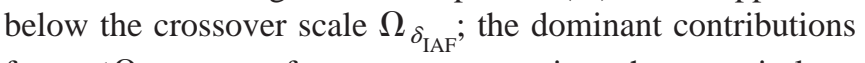
for $\omega \ll \Omega_{\delta_{\text {IAF }}}$ come from some rare regions that contain long finite segments locally in the "metallic" phase.

We begin by providing a rough estimate of these contributions to $\sigma^{\prime}(\omega)$ : In our sample, consider a (large) region of length $L$ locally in the RS phase; the number density of such regions is roughly $p^{L}$, with some $p<1$ (which depends on the distance from the transition). If these regions are effectively isolated from the rest of the system, the average power absorption per spin in each such region is proportional to the finite-size conductivity calculated in the Appendix:

$$
W=L \sigma_{\mathrm{RS}}^{\prime}(\omega, L) \sim L^{3 / 2} \exp \left(-c|\ln \omega|^{2} / L\right),
$$

where we have assumed that $L$, although large, satisfies $L$ $\ll|\ln \omega|^{2}$ (this assumption will turn out to be self-consistent). The total power absorbed in the sample is then obtained by summing over all such regions:

$$
\sigma^{\prime}(\omega) \sim \int d L p^{L} L^{3 / 2} \exp \left(-c|\ln \omega|^{2} / L\right) .
$$

Evaluating this integral by a saddle-point method, we find that the lengths that dominate are of order $|\ln \omega|$ (our assumption about the lengths is thus valid), and arrive at the following estimate

$$
\sigma^{\prime}(\omega) \sim \omega^{\alpha}|\ln \omega|^{2},
$$

where $\alpha=\alpha\left(\delta_{\mathrm{IAF}}\right)>0$ is a continuously varying exponent vanishing at the transition. While this argument is suggestive, we find it more convincing ${ }^{23}$ to take an alternative route based on the scaling picture we have developed earlier for the IAF phase, which has the added advantage of allowing us to relate the exponent $\alpha$ to the dynamical exponent $z\left(\delta_{\mathrm{IAF}}\right)$. This is what we turn to next.

We have already seen that the most numerous low-energy excitations in the IAF Griffiths phase are domain walls, with the integrated density of states $n_{\omega} \sim \omega^{1 / I_{\mathrm{IAF}}}$. Such classical Ising excitations, however, do not contribute to the dynamical conductivity. The dominant contributions come from IAF-ordered clusters of lengths $L \sim \Gamma_{\omega} \Gamma_{\delta_{\text {IAF }}}$ (i.e., with effective spin-flip couplings of order $\omega$ ) that are isolated from the rest of the system by domain walls with $J^{z} \leq \omega$. Remembering that the number density of such Griffiths regions is $\sim \omega^{2 / 2}$ IAF, and noting that the corresponding "phase lengths" are of order $L \sim|\ln \omega|$, we immediately obtain Eq. (47) with $\alpha=2 / z_{\mathrm{IAF}}$. More formally, we sum over the possible separations of two such domain walls, with the constraint that the typical IAF-ordered region isolated by the two has significant spin fluctuations at the characteristic frequency $\omega$ :

$$
\sigma^{\prime}(\omega) \sim \frac{n_{\omega}^{2}}{\omega} \int d L \omega^{2} L^{2} \delta\left(\omega-\Omega_{0} e^{-c_{x} L}\right) .
$$

We thus obtain for the dynamical conductivity in the IAF phase

$$
\sigma^{\prime}(\omega)=\mathcal{K}_{\mathrm{IAF}} l_{v}\left(\omega / \Omega_{0}\right)^{2 / \mathrm{IAF}_{\mathrm{IAF}}} \ln ^{2}\left(\Omega_{0} / \omega\right),
$$

where $\mathcal{K}_{\mathrm{IAF}}$ is a numerical prefactor that depends continuously on $\delta_{\mathrm{IAF}}$. The scaling of $\mathcal{K}_{\mathrm{IAF}}$ with $\delta_{\mathrm{IAF}}$ for small $\delta_{\mathrm{IAF}}$ can be obtained by demanding consistency with the offcritical scaling form for the conductivity

$$
\sigma^{\prime}(\omega)=\mathcal{K}_{\mathrm{RS}} l_{v} \ln \left(\Omega_{0} / \omega\right) \Sigma_{\mathrm{IAF}}\left(\Gamma_{\omega} / \Gamma_{\delta_{\mathrm{IAF}}}\right),
$$

which immediately implies that $\mathcal{K}_{\mathrm{IAF}} \sim \delta_{\mathrm{IAF}}^{(2-\psi) / \lambda} \sim z_{\mathrm{IAF}}^{-1}$.

\section{Dynamical conductivity in $R D$ phases}

We now calculate the dynamical spin conductivity in the $X X$ and $X X X$ random dimer phases. Here, the same singlet RG can be employed all the way across the crossover scale $\Gamma_{\delta} \equiv 1 /|\delta|$, and into the energy regime of a well-developed $\mathrm{RD}$ phase. The dynamical conductivity is given by the same 
expression (42) as for the RS states: we simply add contributions from the even $\left(P^{\mathrm{e}}\right)$ and the odd $\left(P^{\mathrm{o}}\right)$ bonds in complete analogy with the calculation of the dynamic structure factor. Using the scaling solutions of Ref. 6, it is quite simple to calculate the full scaling function for the dynamical conductivity

$$
\sigma^{\prime}(\omega, \delta)=\mathcal{K}_{\mathrm{RS}} l_{v} \ln \left(\Omega_{0} / \omega\right) \Sigma_{\mathrm{RD}}\left[|\delta| \ln \left(\Omega_{0} / \omega\right)\right] .
$$

Here, we restrict ourselves to noting that $\Sigma_{\mathrm{RD}}(x) \sim$ const for $x \ll 1$, while for $x \gg 1, \Sigma_{\mathrm{RD}}(x)$ scales as $\Sigma_{\mathrm{RD}}(x) \sim x e^{-2 x}$. Thus, at frequencies $\omega$ well below the crossover scale $\Omega_{\delta}$, we have

$$
\sigma^{\prime}(\omega)=\mathcal{K}_{\mathrm{RD}} l_{v}\left(\omega / \Omega_{0}\right)^{1 / z_{\mathrm{RD}}} \ln ^{2}\left(\Omega_{0} / \omega\right),
$$

with the numerical prefactor $\mathcal{K}_{\mathrm{RD}} \sim|\delta|$ and the dynamicalexponent $z_{\mathrm{RD}} \sim|\delta|^{-1}$ for small $|\delta|$. We can now interpret this form directly in terms of the rare regions that dominate the conductivity: Assume, for concreteness, that $\delta>0$, i.e., that the even bonds are dominating; the main contribution to the dynamical conductivity at frequency $\omega \ll \Omega_{\delta}$ then comes from the even bonds with effective $\widetilde{J}_{\mathrm{e}}=\omega$. Such weak even bonds are generated only across rare long regions that are locally in the opposite dimerized phase, and these are precisely the regions that dominate the low-energy density of states and thus determine the dynamical exponent $z_{\mathrm{RD}}(\delta)$; this explains the factor $\omega^{1 / z_{\mathrm{RD}}}$ in Eq. (52). Moreover, all such bonds have a well-defined length proportional to $\ln \left(\Omega_{0} / \omega\right)$, which explains the $\ln ^{2}\left(\Omega_{0} / \omega\right)$ in Eq. (52).

\section{Perspective: Spinless interacting fermions with particle-hole symmetric disorder}

To put these transport properties in perspective, we recall that the spin-1/2 XXZ chain is equivalent, via the usual Jordan-Wigner transformation, to a system of spinless interacting fermions with particle-hole symmetric disorder. More specifically, we write the spin operators $s_{j}^{ \pm} \equiv s_{j}^{x} \pm i s_{j}^{y}$ in terms of fermion creation (annihilation) operators $c_{j}^{\dagger}\left(c_{j}\right)$ as

$$
\begin{aligned}
& s_{j}^{+}=\prod_{j^{\prime}<j}\left(1-2 n_{j^{\prime}}\right) c_{j}^{\dagger}, \\
& s_{j}^{-}=\prod_{j^{\prime}<j}\left(1-2 n_{j^{\prime}}\right) c_{j},
\end{aligned}
$$

while $s_{j}^{z}=n_{j}-1 / 2$ (here $n_{j} \equiv c_{j}^{\dagger} c_{j}$ is the fermion number operator at site $j$ ). In this language, $\mathcal{H}_{X X Z}$ can be written as

$$
\mathcal{H}=\sum_{j=1}^{L-1}\left[t_{j}\left(c_{j+1}^{\dagger} c_{j}+c_{j}^{\dagger} c_{j+1}\right)+V_{j}\left(n_{j}-1 / 2\right)\left(n_{j+1}-1 / 2\right)\right],
$$

with $t_{j}=J_{j}^{\perp} / 2$ and $V_{j}=J_{j}^{z}$. The coupling $J^{z}$ thus controls the strength of the nearest-neighbor particle-hole-symmetric repulsive interaction between the fermions. The IAF phase that obtains for large $J^{z}$ corresponds to a charge-density-wave state stabilized by interactions. In the absence of interactions ( $X X$ chain) we obtain a free-fermion random-hopping problem at zero chemical potential.
This free-fermion problem has been extensively studied in the past, and is known to have rather unusual localization properties due to the additional particle-hole symmetry present. ${ }^{24,25}$ For instance, an elementary calculation immediately reveals that the zero-temperature average Landauer conductance $\left[g_{L}\right]_{\text {av }}$ of a finite segment of length $L$ connected to perfect leads scales as $\left[g_{L}\right]_{\mathrm{av}} \sim 1 / \sqrt{L}$, in sharp contrast to the usual exponentially-localized behavior in one dimension; the corresponding conductivity, of course, scales as $\sqrt{L}$. Now, the strong-disorder RG predicts that lengths scale as the square of the logarithm of the energy scale in the lowenergy effective theory describing the $X X$-RS state; our result for the dynamical conductivity is thus consistent with the elementary Landauer calculation (see also our explicit finitesize scaling calculations in the Appendix). ${ }^{26}$ Notice, however, that our approach is not limited to the noninteracting case. It allows us to reliably treat the effects of interactions, and follow the dynamical conductivity through a "metalinsulator" transition that is driven by strong interactions in the presence of strong disorder.

\section{Numerical study of the dynamical conductivity at the $X X$ fixed point}

At the $X X$ point, the Hamiltonian Eq. (54) describes noninteracting fermions with random hopping amplitudes, and we are essentially faced with the problem of finding the lowenergy eigenvalues and eigenstates of the corresponding single-particle Hamiltonian (an $L \times L$ matrix operator) $\mathbf{H}$ $=\sum_{j=1}^{L-1} t_{j}(|j+1\rangle\langle j|+| j\rangle\langle j+1|)$, which defines the Schrödinger equation for this problem. Any fermionic state can then be represented as a Slater determinant of the corresponding (normalized) single-particle eigenstates $\left|\phi_{\mu}\right\rangle$ with eigenenergies $\epsilon_{\mu}$. In the single-particle language, the Kubo formula for the conductivity $\sigma^{\prime}(\omega)$ at zero chemical potential and at a finite temperature $T$ reads

$$
\begin{aligned}
\sigma^{\prime}(\omega)= & \frac{1}{\omega L} \sum_{\mu_{1}, \mu_{2}}\left|\left\langle\phi_{\mu_{2}}\left|\sum_{j} \mathbf{T}(j)\right| \phi_{\mu_{1}}\right\rangle\right|^{2} \\
& \times\left[f\left(\epsilon_{\mu_{1}}\right)-f\left(\epsilon_{\mu_{2}}\right)\right] \delta\left(\omega-\epsilon_{\mu_{2}}+\epsilon_{\mu_{1}}\right),
\end{aligned}
$$

where $\mathbf{T}(j) \equiv i t_{j}(|j\rangle\langle j+1|-| j+1\rangle\langle j|)$ is the current operator on the link $j$ and $f(\epsilon) \equiv 1 /\left(e^{\epsilon / T}+1\right)$. (This version of the Kubo formula will also prove useful when we analyze the full temperature dependence of the dynamical conductivity in Sec. VI.)

Here, we test the $T=0$ predictions by evaluating $\sigma^{\prime}(\omega)$ using exact numerical diagonalization of finite systems. The results of such calculations for system sizes $L=128,256$, and 512 with the hopping amplitudes $t_{j}$ drawn independently from a uniform distribution over $[0,1]$ are shown in Fig. 6, where we have averaged over 100000 samples for each $L$. In an infinite sample we expect the conductivity to diverge logarithmically, but with the system sizes studied here, we cannot quite probe this infinite-sample regime $1 \ll \ln \left(\Omega_{0} / \omega\right)$ $\ll \sqrt{L}$, rather, we are in the regime $1 \ll \ln \left(\Omega_{0} / \omega\right) \lesssim \sqrt{L}$. Nevertheless, the numerical results of Fig. 6 clearly show that the dynamical conductivity increases as the frequency is lowered 


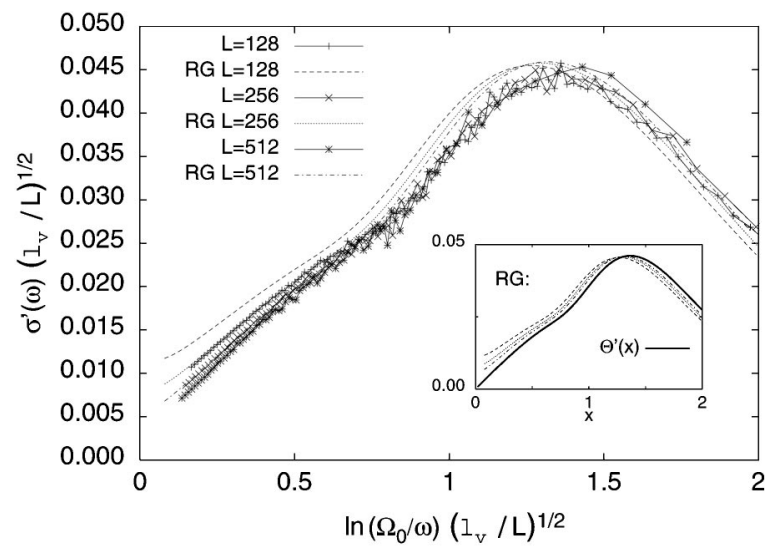

FIG. 6. Scaling plot of the dynamical conductivity $\sigma^{\prime}(\omega, L)$ at the $X X$ point for finite systems of sizes $L=128,256$, and 512, with free boundary conditions, calculated by exact numerical diagonalization and, for exactly the same systems, by the finite-size RG analysis of the Appendix. Free-fermion hopping amplitudes $t_{j}$ are drawn independently from a uniform distribution over $[0,1]$, and the (bare) phase lengths are set to $l_{j}=1$. We used $\Omega_{0}=2$ corresponding to the initial energy cutoff in the equivalent spin system; also, we used $l_{v}=1$ corresponding to the microscopic length scale in the problem. The agreement of the RG predictions with the results of the exact diagonalization is fairly good (given the not-sostrong initial disorder), and the dynamical conductivity is indeed increasing all the way to the (finite-size) crossover scale $\ln \left(\Omega_{0} / \omega\right.$ ) $\sim \sqrt{L / l_{v}}$. Note that with the sizes studied, we can only partially access the bulk scaling regime $\ln \left(\Omega_{0} / \omega\right) \ll \sqrt{L / l_{v}}$, in which we expect $\sigma^{\prime}(\omega, L) \sim \ln \left(\Omega_{0} / \omega\right)$ and which on the plot is toward the left of the horizontal axis. Also note that in the opposite regime $\ln \left(\Omega_{0} / \omega\right)>\sqrt{L / l_{v}}$, unlike in the bulk regime, $\sigma^{\prime}(\omega, L)$ is not selfaveraging; in this regime, the plotted average over different samples represents roughly the distribution of the lowest gap in the system. Inset shows how the $L \rightarrow \infty$ scaling form (thick line) is approached by the finite-size $\sigma^{\prime}(\omega, L)$ calculated from the RG for the given initial conditions (the lines plotted here are the same as in the main panel). Note that the vertical scale is set by the (numerical) prefactor of the scaling function in the bulk scaling regime [where $\left.\sigma^{\prime}(\omega) \approx \frac{7}{180} \ln \left(\Omega_{0} / \omega\right)\right]$.

all the way to the crossover scale $\ln \left(\Omega_{0} / \omega\right) \sim \sqrt{L}$, thus supporting our claim that $\sigma^{\prime}(\omega)$ diverges logarithmically at low frequencies.

For a more detailed test of our theoretical results, we need to quantitatively analyze the effects of a finite system size on our predictions for the dynamical conductivity. The calculation is summarized in the Appendix. Here, we only note that this analysis allows us to write the following scaling form for the conductivity:

$$
\sigma^{\prime}(\omega, L)=l_{v} \ln \left(\Omega_{0} / \omega\right) \Theta\left[l_{v} \ln ^{2}\left(\Omega_{0} / \omega\right) / L\right] ;
$$

the scaling function $\Theta$ is characterized in the Appendix, and the above result is expected to hold for large enough $L$ and $\ln \left(\Omega_{0} / \omega\right)$ [with no restrictions on the ratio $\ln ^{2}\left(\Omega_{0} / \omega\right) / L$ ]. However, the numerical results cannot be compared directly with this scaling result since it assumes that the distribution of bond lengths has reached the form characteristic of the $X X$ fixed point, which is not the case for the sizes that we can diagonalize numerically: the "length part" of the distribution $P(\zeta, l \mid \Gamma)$ is still evolving toward the corresponding scaling form from the initial condition $P\left(\zeta, l \mid \Gamma_{I}\right)=e^{-\zeta} \delta(l-1)$. Nevertheless, we can compare the results of the exact numerical diagonalization with the (formal) predictions of the RG for the same systems. This can be done by either running the RG on the same samples or by evaluating the analytical (within the RG) expression, given by the inverse Laplace transform Eq. (A9) for these initial conditions. In Fig. 6, we compare the RG result obtained in this manner with the numerically evaluated conductivity. Given that the initial disorder is not very strong, the agreement of the RG predictions with the $\sigma^{\prime}(\omega, L)$ from the exact diagonalization is fairly good.

\section{E. On validity of results}

So far, our calculations have relied on the leading-order renormalizations of the spin operators; in this subsection we will try to justify validity of this approximation. We will not address the corresponding question for the RG itself because this has been analyzed with great care in Refs. 6 and 4, and we have nothing to add here. Instead, we focus on issues specific to our calculation of dynamical quantities, and therefore not addressed as such in previous work. Here, we provide a (partial) justification of our leading-order results by analyzing the effects of the first corrections to the leadingorder expressions for the renormalized operators; this can be done consistently within the framework of the RG approach. Any consistent analysis of further corrections would require that we also consider higher-order corrections to the RG rules themselves, and we stop well short of doing that. ${ }^{27}$

As an illustrative example, we consider the dynamic structure factor in the RS states. Our leading-order calculations used only the zeroth-order result for the renormalized spin operators. The renormalized operators can also be easily worked out to first order; these were considered in the discussion of typical correlations in Ref. 4 . When a pair of spins 2 and 3 connected by a strong bond is frozen into a singlet state, the neighboring spin operators $\vec{s}_{1}$ and $\vec{s}_{4}$ do not change even to first order, while the spin operators $\vec{s}_{2}$ and $\vec{s}_{3}$ renormalize to

$$
\begin{gathered}
\left(s_{2}^{z}\right)^{\mathrm{eff}}=-\left(s_{3}^{z}\right)^{\mathrm{eff}}=-\frac{J_{1}^{z}}{2 J_{2}^{\perp}} s_{1}^{z}+\frac{J_{3}^{z}}{2 J_{2}^{\perp}} s_{4}^{z}, \\
\left(s_{2}^{+}\right)^{\mathrm{eff}}=-\left(s_{3}^{+}\right)^{\mathrm{eff}}=-\frac{J_{1}^{\perp}}{J_{2}^{\perp}+J_{2}^{z}} s_{1}^{+}+\frac{J_{3}^{\perp}}{J_{2}^{\perp}+J_{2}^{z}} s_{4}^{+} .
\end{gathered}
$$

Thus, the decimated spins $\vec{s}_{2}$ and $\vec{s}_{3}$ obtain small "components" of order $J_{1} / J_{2}, J_{3} / J_{2}$, onto the neighboring spins $\vec{s}_{1}$ and $\vec{s}_{4}$. As we run the RG and renormalize down to scale $\Gamma$, the system consists of $n_{\Gamma}$ active spins per unit length, separated from each other by "dead" regions (with lengths of order $\Gamma^{2}$ ) of decimated spins. Each decimated spin $r$ in the dead region between two remaining active spins $j$ and $k$ (where $j$ and $k$ are nearest neighbors in the effective Hamiltonian at scale $\Gamma$ ) will have some components $C_{r j}$ and $C_{r k}$ 
on spins $\vec{s}_{j}$ and $\vec{s}_{k}$. From the point of view of calculating the spectral sum Eq. (4), each active spin $j$ acquires some ( $k$-dependent) effective moment $\tilde{\mu}_{j}(k)$ coming from all decimated spins with nonzero components on $\vec{s}_{j}$ :

$$
\tilde{\mu}_{j}(k)=1+\sum_{i_{1}<r<i_{2}}^{\prime} C_{r j} e^{i k(r-j)}
$$

where the sum is over all previously decimated spins $r$ between the effective neighbors $i_{1}$ and $i_{2}$ of the spin $j, i_{1}<j$ $<i_{2}$. The components $C_{r j}$ are simply the ground-state correlations $^{28}\left\langle s_{r} s_{j}\right\rangle$; such typical correlators decay as a stretched exponential $-\left[\ln C_{r j}\right]_{\mathrm{av}} \sim|r-j|^{1 / 2}$. Note that the characteristic length scale for this decay is the microscopic length scale $l_{v}$. It is thus clear that the sum over $r$ in Eq. (58) converges quickly, and the renormalization of the moment $\tilde{\mu}_{j}$ away from its bare value of 1 comes mainly from the nearby spins that were decimated early in the RG. This renormalization is of order one, but only weakly $k$ dependent.

We now analyze the consequences of this renormalization of the moments for the two scaling forms of the dynamic structure factor derived earlier in the limit of low frequencies, one in the vicinity of $k=\pi / a$, and the other in the vicinity of $k=0$. First, consider $k=\pi / a+q$, with $|q| \ll l_{v}^{-1}$. For such small values of $q$, we can neglect the $q$ dependence of the moments and evaluate them at $k=\pi / a$. To evaluate the spectral sum Eq. (4), we need to add up the contributions coming from the strong bonds at scale $\Omega_{\text {final }}$. Each strong bond contributes $\left|\tilde{\mu}_{L}+\tilde{\mu}_{R} e^{i q l}\right|^{2}$, where $\mu_{L}$ and $\mu_{R}$ are the moments (evaluated at $k=\pi / a$ ) of the two spins connected by this strong bond. We can now proceed in two steps: First, we fix $l$ and average over the moments of all strong bonds with a given length. This gives us a quantity $c_{1}+c_{2} \mid 1$ $+\left.e^{i q l}\right|^{2}$ that we now need to average over the length distribution of the strong bonds; here $c_{1}$ and $c_{2}$ are now some fixed numbers of order one, since we expect that the main renormalization of each moment comes from few nearby spins and is roughly independent of the lengths of the adjoining bonds. Thus, we see that Eq. (24), with $\Phi$ given by Eq. (25), indeed describes the dynamic structure factor for $k$ close to $\pi / a$ and a fixed low $\omega$; the higher-order corrections renormalize the overall amplitude by a factor of order one, and also produce an "incoherent" background of a comparable strength that depends only weakly on $k$ (i.e., that changes significantly only when $k$ is changed by an amount of order $l_{v}^{-1}$ ).

For $k \ll l_{v}^{-1}$ (i.e., in the scaling regime near $k=0$ ), the discussion is very similar; each strong bond contributes $\mid \tilde{\mu}_{L}$ $-\left.\tilde{\mu}_{R} e^{i k l}\right|^{2}$, where the moments are now evaluated at $k=0$. This again gives us a quantity $c_{1}+c_{2}\left|1-e^{i k l}\right|^{2}$ to be averaged over the length distribution of the strong bonds. In general (away from the $X X X$ point), we now have to consider the $S^{z z}(k, \omega)$ component separately from the $S^{+-}(k, \omega)$ component, since the total $s_{\text {tot }}^{z}$ conservation constrains the constant $c_{1}$ to be identically zero for the case of $S^{z z}(k, \omega)$. Thus, in the case of $S^{z z}(k, \omega)$, higher-order corrections only produce an order one renormalization to the overall scale of our scaling result Eq. (26); of course, there will be additional corrections, but these will vanish faster than the scaling result in the low-frequency limit. In the case of $S^{+-}(k, \omega)$, an inspection of the renormalization rules Eq. (57) shows that to this order $c_{1}$ will be zero for $S^{+-}(k, \omega)$ as well, even in the absence of full Heisenberg symmetry; however, this is not expected to be true in general (to all orders), and we expect a small but nonzero background to be present in the general case. Thus, $S^{+-}(k, \omega)$ near $k=0$ will in general consist of two parts: the scaling part given by Eq. (57) with an order one nonuniversal overall scale (this part vanishes as $\sim k^{2}$ for small $k$ ), and a nonscaling, weakly $k$-dependent additive background of the same order as the scaling part.

The above arguments typify the general logic behind our justification of the leading-order results for all of our calculations; in some cases such a program can be carried out analytically, ${ }^{29}$ while in other cases we have to be satisfied with arguments like the ones presented above. Such arguments can also be bolstered by numerically implementing the higher-order operator renormalizations to calculate corrections within the RG to our leading order results [indeed, we have confirmed that such a numerical check for $S(k, \omega)$ in the Heisenberg model is in qualitative agreement with the arguments presented above].

\section{TRANSPORT IN STRONGLY RANDOM SPIN-1 CHAINS}

\section{A. Singlet RG description of the phases: A review}

The strong-randomness quantum critical point, which controls the transition from the Haldane state to the random singlet state in the spin-1 chains, and the immediate vicinity of this critical point, can be analyzed by a somewhat extended RG procedure introduced in Refs. 18 and 5, or by a variant of the same used in Ref. 8.

The basic idea is to replace the original spin- 1 chain by an effective model that is argued to describe the low-energy physics of the original system. As we shall see later, this effective model can be made plausible by thinking in terms of a bond-diluted chain (it is also possible to arrive at essentially the same model by starting with a random antiferromagnetic spin-1 chain and using the approximate ${ }^{30} \mathrm{RG}$ procedure of Ref. 8). This effective model is written entirely in terms of spin-1/2 degrees of freedom coupled by nearestneighbor Heisenberg exchange couplings. All even bonds are always antiferromagnetic and are drawn from an appropriate distribution of positive bonds, while odd bonds can be of either sign and are drawn from a different distribution.

This effective model can be analyzed using the extension of the singlet RG introduced in Refs. 18 and 5. One begins by looking for the largest antiferromagnetic bond in the system, say $J_{2}$ connecting spins 2 and 3; this defines our bare energy-cutoff $\Omega_{0}$. Further analysis can be split into three cases: (i) If the bonds adjacent to the largest AF bond are smaller in magnitude, the two spins are frozen into a singlet state and an effective coupling $\widetilde{J}_{14}$ is generated between spins 1 and 4 exactly as in the singlet RG for the spin-1/2 
chain. (ii) If both the adjacent bonds are larger in magnitude than $J_{2}$, then spins 1 and 2 and spins 3 and 4 are first combined to make effective spin-1 objects (since in this case $J_{1}$ and $J_{3}$ are necessarily ferromagnetic), and these effective spin-1 degrees of freedom are then frozen into a singlet state, generating an effective coupling $\widetilde{J}_{05}=4 J_{0} J_{4} / 3 J_{2}$ between spins 0 and 5. (iii) If only one of the adjacent bonds, say $J_{3}$, is larger in magnitude than $J_{2}$, then spins 3 and 4 are first combined into an effective spin-1 object. The system is then frozen into the subspace in which spin 2 and this effective spin-1 object are coupled together to form an effective spin$1 / 2$ object that we label as $s_{2}$ for consistency of notation. The corresponding renormalized couplings are given as $\widetilde{J}_{12}$ $=-J_{1} / 3$ and $\widetilde{J}_{25}=2 J_{4} / 3$. This procedure is now iterated with the energy-cutoff $\Omega$ being gradually reduced. It is important to note that there is no inconsistency in leaving ferromagnetic bonds $J<-\Omega$ untouched that are not adjacent to any antiferromagnetic bonds at the cutoff scale; we could equally well have combined all pairs of spins connected by such strong ferromagnetic bonds into effective spin-1 objects at the cost of cluttering up our notation.

A detailed analysis of this iterative procedure can be summarized as follows: ${ }^{18,5,8}$ Let $\Gamma \equiv \ln \left(\Omega_{0} / \Omega\right)$ and let $n_{\Gamma}$ be the fraction of active spins at $\log$-cutoff $\Gamma$. For the even bonds, we introduce the distribution $P(\zeta \mid \Gamma)$ of the corresponding logarithmic couplings $\zeta \equiv \ln (\Omega / J)$. For the odd bonds, let $N(\Gamma)$ be the fraction of odd bonds at scale $\Gamma$ that are strongly ferromagnetic with $J<-\Omega$; for large $\Gamma$, the remainder of the odd bonds are symmetrically distributed about zero and are therefore described by a distribution for $|J|$ that we characterize by the distribution $Q(\zeta \mid \Gamma)$ of the corresponding logarithmic couplings $\zeta \equiv \ln (\Omega /|J|)$. When $W$, the width of the distribution of the log-exchanges in the original spin-1 Hamiltonian Eq. (6), exceeds a critical value $W_{c}$, the system is in a spin-1 random singlet phase. In the language of the spin-1/2 effective model, this RS phase is described by a fixed point with $P(\zeta \mid \Gamma)=\Gamma^{-1} e^{-\zeta / \Gamma}, N(\Gamma)=1, n_{\Gamma} \sim 1 / \Gamma^{2}$, and $Q(\zeta \mid \Gamma)=Q_{0} e^{-Q_{0} \zeta}$ for large $\Gamma\left[Q_{0}\right.$ is some nonuniversal $\mathrm{O}(1)$ number]. As $W$ is decreased, the system undergoes a quantum phase transition to the so-called gapless Haldane phase; both the quantum critical point and the GH phase in the vicinity of it are still controlled by strong-disorder fixed points. At the critical fixed point (which is an infinitedisorder fixed point) we have $P(\zeta \mid \Gamma)=Q(\zeta \mid \Gamma)$ $=2 \Gamma^{-1} e^{-2 \zeta / \Gamma}, n_{\Gamma} \sim 1 / \Gamma^{3}$, and $N(\Gamma)=1 / 2$. The GH phase in the vicinity of the quantum critical point is controlled by a line of fixed points; each point on this line is characterized by some constant $P_{0}$ (which depends on the strength of disorder $W)$. At a point labeled by $P_{0}$, we have $P(\zeta \mid \Gamma)$ $=P_{0} e^{-P_{0} \zeta}, \quad Q(\zeta \mid \Gamma)=Q_{0}(\Gamma) e^{-Q_{0}(\Gamma) \zeta} \quad$ where $\quad Q_{0}(\Gamma)$ $\sim e^{-P_{0} \Gamma}, N(\Gamma) \rightarrow 0$, and $n_{\Gamma} \sim P_{0}^{3} e^{-P_{0} \Gamma}$. The continuously varying $P_{0}(W)$ vanishes at the transition as $P_{0} \sim\left(W_{c}\right.$ $-W)^{\nu / 3}$, where $\nu$ is the correlation length exponent obtained in Refs. 18 and 5; the GH phase is thus similar to the dimerized phases of the spin- $1 / 2$ chains.

\section{B. Spin transport}

\section{Doing calculations in the effective model}

Before we calculate anything, we need to describe how we think about the spin transport in this case. This is some- what nontrivial, for we are working in an effective model of spin-1/2 degrees of freedom, and some thought is required to decide what is the correct quantity to calculate.

For this, we go back for a moment to the original random spin- 1 chain and review an intuitive construction that leads to the effective model in terms of spin-1/2 variables only. Consider the case of dilute randomness, ${ }^{18}$ that is, consider a uniform spin-1 chain with a small fraction of very weak bonds that effectively break the chain into pure finitesegments weakly coupled with each other. The low-energy effective degrees-of-freedom of such a segment are two halfspins localized near the two edges of the segment-these are the spin-1/2's of the effective model. The coupling of the edge spins on neighboring segments is given roughly by the original coupling of the two segments, and is always antiferromagnetic - these are the even bonds of the effective model. On the other hand, the coupling of the two edge-spins of the same segment can be either antiferromagnetic or ferromagnetic depending on whether the length of the segment is even or odd; these couplings are represented by the odd bonds in the effective model.

We now need to express dynamical properties of the system in terms of these effective spin-1/2 degrees of freedom. In particular, we want to analyze the low-frequency power absorption when an oscillating magnetic field with a uniform gradient is applied to the system; this will give us the dynamical conductivity $\sigma^{\prime}(\omega)$. Since the magnetic field couples to the conserved "charge" in the system, the corresponding current operators that we need to use when working out the Kubo formula for the effective model are uniquely determined by spin conservation: The current operator on the odd bonds connecting the edge half-spins $\vec{s}_{1}$ and $\vec{s}_{2}$ of the same segment (which represents the total-spin current operator of this segment) is $\vec{\tau}=J_{12} l_{12} \vec{s}_{1} \times \vec{s}_{2}$; here $J_{12}$ is the corresponding effective coupling and $l_{12}$ is some effective phase length that we expect to be given roughly by the length of the segment. Naturally, the current operators on the even bonds connecting the edge half-spins of the neighboring segments have a similar form. (The argument in this case is even simpler: one only needs to know that the true edge spin-1 operator of a segment "projects" onto the corresponding effective edge spin-1/2 operator with an amplitude of order one.) Note that the precise values of the phase lengths in the initial effective model (for the dilute spin-1 chain) are not important, since at still lower energies we expect the distributions of couplings and the corresponding bond lengths to approach some universal distributions characteristic of the appropriate fixed point.

\section{Dynamical conductivity}

Having identified the appropriate current operators in the effective problem, we now work out the rules that govern their renormalization in the $\mathrm{RG}$ scheme used to analyze this effective model. As in the spin-1/2 case, and as discussed above, we write the part of the total current operator [in the spectral sum Eq. (5)] that is associated with a given bond $(j, j+1)$ in the form $l_{j} \vec{\tau}_{j}$ where $\vec{\tau}_{j}$ is the usual bond operator $\vec{\tau}_{j}=J_{j} \vec{s}_{j} \times \vec{s}_{j+1}$ and $l_{j}$ is the appropriate phase length. We 
can then follow renormalizations of the needed operators by keeping track of the phase lengths, in addition to the various bond-strengths. Unlike the spin-1/2 chains, these phase lengths need not equal the physical distances between the corresponding spins; in fact, even the physical position of an effective half-spin often cannot be specified unambiguously, as, for example, when this half-spin appears as an effective doublet formed by combining (via a strong AF bond) an effective spin-1 (which is an intermediate construction in the Hyman-Yang RG rules) and a neighboring spin-1/2. In such cases, our rules can actually be used to assign some meaning to the physical position of such an effective half-spin.

The rules for the phase lengths can be easily stated: In the cases (i) and (ii), when in the final step we form a singlet from either two spin-1/2 objects or two spin- 1 objects, the phase length of the new effective bond is simply the sum of the phase lengths of all the bonds that are eliminated. In the case (iii) the phase lengths associated with effective bonds $\widetilde{J}_{12}$ and $\widetilde{J}_{25}$ are $\tilde{l}_{12}=l_{1}+(4 / 3) l_{2}+(2 / 3) l_{3}$ and $\tilde{l}_{25}=l_{4}$ $-(1 / 3) l_{2}+(1 / 3) l_{3}$, respectively.

The rules for the phase lengths in the case (iii) are somewhat unusual; for example, negative phase lengths can be produced. Note, however, that there are many factors that prevent this from happening too often, and the phase lengths will in many instances coincide with the corresponding geometrical lengths: decimations in the cases (i) and (ii) tend to "correct" deviations of the phase lengths from the geometrical lengths, and in both the RS and GH phases there are simply no decimations of type (iii) at low-enough energies. Also, the lengths $l_{2}$ and $l_{3}$ in the above rule for the case (iii) are the lengths of the strong bonds that are eliminated and are therefore usually smaller than the lengths $l_{1}$ and $l_{4}$ of the more typical bonds. Finally, one can argue generally that the phase positions of the spins as dictated by the phase lengths have to agree, at least roughly, with their geometrical positions as inferred from the order of the (remaining) spins in the chain (i.e., from the spin labels). ${ }^{31}$ All of this implies that the phase lengths are roughly given by the geometrical distances between the spins; in particular their scaling with $\Gamma$ is given by the inverse of the density of the remaining spins, $l \sim n(\Gamma)^{-1}$.

We can now immediately deduce behavior of the dynamical conductivity in the different phases exactly as in our previous calculations for the spin-1/2 model; as the method remains the same, and the relevant details about the statistics of the fixed point Hamiltonians have already been summarized, we merely state our results.

In the RS phase the same result Eq. (42) applies, as is true for an RS state of an arbitrary- $S$ spin chain at a strongenough randomness.

At the critical point separating the RS phase from the GH phase, we find

$$
\sigma^{\prime}(\omega)=\mathcal{K}_{\mathrm{HY}} l_{v} \ln ^{2}\left(\Omega_{0} / \omega\right),
$$

which is a stronger divergence than in the RS phase (note that this difference from the result in the RS states can be traced to the fact that the density of the remaining spins behaves as $n_{\Gamma} \sim 1 / \Gamma^{3}$ at the critical point, in contrast to the $1 / \Gamma^{2}$ decay of the corresponding quantity in the RS states).

Finally, in the GH phases parametrized by $P_{0}$ we find

$$
\sigma^{\prime}(\omega)=\mathcal{K}_{\mathrm{GH}} l_{v}\left(\omega / \Omega_{0}\right)^{1 / z_{\mathrm{GH}}} \ln ^{2}\left(\Omega_{0} / \omega\right),
$$

where we have introduced the continuously varying dynamical-exponent $z_{\mathrm{GH}} \equiv P_{0}^{-1}$, and $\mathcal{K}_{\mathrm{GH}}$ is an order-one numerical prefactor that goes to a constant as $W \rightarrow W_{c}$. [Note that the factor $\ln ^{2}\left(\Omega_{0} / \omega\right)$ appears for exactly the same reasons as in the RD phases of the spin- $1 / 2$ chains: the lengths of the singlets that are decimated at scale $\omega$ are roughly $\sim \ln \left(\Omega_{0} / \omega\right)$.]

\section{DYNAMICS IN THE RANDOM TRANSVERSE-FIELD ISING CHAIN}

\section{A. Strong-disorder RG description of the phases: A review}

The strong-randomness cluster RG of Ref. 6, from which the low-energy long-distance behavior of a system near the critical point $(|\delta| \ll 1)$ can be obtained, proceeds as follows: One finds the largest coupling in the system, with energy $\Omega_{0} \equiv \max \left\{h_{j}, J_{j}\right\}$. If the largest coupling is a field, say $h_{2}$ on spin 2, this spin is frozen into the $\sigma_{2}^{x}=+1$ ground state of the local field term and is eliminated from the system leaving an effective coupling $\widetilde{J}_{13}=J_{12} J_{23} / h_{2}$ between the neighboring spins 1 and 3 . If the largest coupling is an interaction, say $J_{12}$ between spins 1 and 2, the two spins are combined into one new spin - a cluster-with an effective spin variable $\tilde{\sigma}_{(12)}$ (representing the two classical minimum-energy states $\left.\sigma_{1}^{z}=\sigma_{2}^{z}= \pm 1\right)$ and an effective transverse field $\tilde{h}_{(12)}$ $=h_{1} h_{2} / J_{12}$; the couplings of this new spin to the neighbors remain unchanged to leading order. Each such cluster $c$ has a moment $\tilde{\mu}_{c}$ given by the number of initial spins in the cluster; when two clusters are combined to form a bigger cluster, their moments add: $\tilde{\mu}_{(12)}=\mu_{1}+\mu_{2}$. This procedure is now iterated with the energy-cutoff $\Omega \equiv \max \left\{\widetilde{h}_{j}, \widetilde{J}_{j}\right\}$ of the new, effective Hamiltonian being gradually lowered.

A detailed analysis of this procedure was given in Ref. 6, of which a summary follows: Define the log-couplings $\beta_{i}$ $\equiv \ln \left(\Omega / h_{i}\right), \quad \zeta_{i} \equiv \ln \left(\Omega / J_{i}\right), \quad$ and also the log-cutoff $\Gamma$ $\equiv \ln \left(\Omega_{0} / \Omega\right)$; also, let $n_{\Gamma}$ be the number-density per unit length of the (remaining) clusters at scale $\Gamma$. The essential feature of the RG near the critical point is that the distributions of the log-couplings $R(\beta \mid \Gamma)$ and $P(\zeta \mid \Gamma)$ become broader and broader as the energy cutoff is lowered; the RG flows are characterized by a special family of scaling solutions with $R(\beta \mid \Gamma)=R_{0}(\Gamma) e^{-R_{0}(\Gamma) \beta} \quad$ and $P(\zeta \mid \Gamma)$ $=P_{0}(\Gamma) e^{-P_{0}(\Gamma) \zeta}$. At the critical point, $\delta=0$, we have $R_{0}(\Gamma)=P_{0}(\Gamma)=1 / \Gamma$; thus the widths of the two distributions grow without limit, and the number density decreases as $n_{\Gamma} \sim 1 / \Gamma^{2}$. Also, magnetic moments of the clusters scale as $\mu \sim \Gamma^{\phi}$, with $\phi=(1+\sqrt{5}) / 2$. In the disordered phase, $\delta>0$, beyond the crossover scale $\Gamma_{\delta} \equiv 1 /|\delta|$, the width of the field distribution saturates, with $R_{0}(\Gamma) \approx 2 \delta$ for $\Gamma \gg \Gamma_{\delta}$, while the width of the bond distribution grows without limit, with $P_{0}(\Gamma) \approx 2 \delta e^{-2 \delta \Gamma}$. In the ordered phase, $\delta<0$, the situation is reversed: $R_{0}(\Gamma) \approx 2|\delta| e^{-2|\delta| \Gamma}$ and $P_{0}(\Gamma) \approx 2|\delta|$ for $\Gamma$ 
$\gg \Gamma_{\delta}$. In both phases, we have $n_{\Gamma} \sim|\delta|^{2} e^{-2|\delta| \Gamma}$. Note also that the clusters that are being eliminated at scale $\Gamma \gg \Gamma_{\delta}$ all have a fairly well-defined length of order $|\delta|^{-1} \Gamma$ and magnetic moment of order $|\delta|^{1-\phi} \Gamma$.

\section{B. Average autocorrelations}

In this section, we obtain the long-time asymptotics of average imaginary-time autocorrelations in the critical region of the RTFIM-we will be heavily using results of Ref. 6 referring to sections in that paper by, e.g., F Sec. IVB. At the end of the section, we compare our predictions with the numerical results ${ }^{17}$ available in the literature.

We consider the local dynamical susceptibilities

$$
\chi_{j j}^{\alpha \alpha}=\sum_{m}\left|\left\langle m\left|\sigma_{j}^{\alpha}\right| 0\right\rangle\right|^{2} \delta\left(\omega-E_{m}\right),
$$

where the sum is over all excited states $|m\rangle$ with excitation energies $E_{m}$, and $\alpha=x$ or $\alpha=z$. The low-frequency behavior of these susceptibilities determines the long-time asymptotics of the corresponding imaginary-time autocorrelation functions

$$
C_{j j}^{\alpha \alpha}(\tau)=\left\langle\sigma_{j}^{\alpha}(\tau) \sigma_{j}^{\alpha}(0)\right\rangle,
$$

with $\alpha=z$ (local-magnetic-moment autocorrelation) or $\alpha$ $=x$ (local-energy autocorrelation); we are considering here only the fluctuating (time-dependent) parts of autocorrelations and will ignore any constant (time-independent) parts (such a constant part in, for example, spin autocorrelation represents a nonzero magnetization density in the system and is a static property). In the following, we simply write $C_{\text {loc }}(\tau)$ for the local magnetic moment autocorrelations $C_{j j}^{z z}(\tau)$ and $C_{\mathrm{loc}}^{e}(\tau)$ for the local-energy autocorrelations $C_{j j}^{x x}(\tau)$ (and similarly for susceptibilities). We first obtain (using our basic strategy) results for average susceptibilities, which can be conveniently defined as

$$
\left[\chi_{\mathrm{loc}}^{\alpha \alpha}\right]_{\mathrm{av}}(\omega)=\frac{1}{L} \sum_{j} \chi_{j j}^{\alpha \alpha}(\omega),
$$

where $L$ is the size of the system (in the thermodynamic limit of $L \rightarrow \infty$ this definition coincides with an ensemble average over disorder realizations). We also consider a semi-infinite chain, $j \geqslant 1$, and calculate average dynamical susceptibilities $\chi_{1}^{\alpha \alpha}$ of the boundary spin $\sigma_{1}$ (in this case, the average is over disorder realizations). These results are then immediately translated to the corresponding statements about the longtime behavior of average autocorrelations. As long as we are interested only in the asymptotic behavior of the average dynamical susceptibilities and autocorrelations, it suffices to use the leading-order results for the renormalization of the corresponding operators. ${ }^{29}$

Before we plunge into the details below, it is worth emphasizing that the calculations in this section are closely related to the discussion in Ref. 6 of static response functions at finite temperature $T$ : Such static response properties are calculated by assuming that all effective degrees of freedom that are present (in the sense of the RG) at energy-scale $T$ contribute freely to the response at this temperature, while in our calculations of the dynamical properties at frequency $\omega$ only the degrees of freedom that are being decimated at scale $\omega$ contribute to the dynamical response at this frequency. (The connection is even more apparent when the dynamical susceptibilities are translated to the imaginary-time autocorrelations, since average autocorrelations at time $\tau$ acquire contributions from all frequencies smaller than $1 / \tau$.) Our aim here is to present a unified approach, within the RG of Ref. 6, to the analytical calculation of such average dynamical properties. Also, these calculations, together with a detailed physical picture developed in Ref. 6 of the phases of the system near the critical point, serve as a valuable guide to our intuition in identifying the relevant Griffiths regions that dominate a particular response; on some occasions in the previous sections (particularly in the IAF phase of spin-1/2 chains), such Griffiths arguments were our only source of information about the behavior of dynamical quantities, and the opportunity to compare such suggestive arguments against the results of controlled calculations is most welcome.

\section{Average local-spin autocorrelation $\left[C_{\mathrm{loc}}\right]_{\mathrm{av}}(\tau)$}

The leading-order renormalizations of the $\sigma^{z}$ spin operators are particularly simple: As long as a given spin $j$ is active, the operator $\sigma_{j}^{z}$ is renormalized to the "spin" operator $\tilde{\sigma}_{c}^{z}$ of the cluster $c$ that the spin $j$ belongs to; when this cluster is decimated, the corresponding operator renormalizes to zero.

To calculate $\left[\chi_{\mathrm{loc}}\right]_{\mathrm{av}}(\omega)$, we run the RG down to energy scale $\Omega_{\text {final }}=\omega / 2$, and rewrite the spectral sum in terms of the degrees of freedom of the renormalized problem; excitations that contribute to this new sum are clearly the $\tilde{\sigma}^{x}$ $=+1$ excitations of the spin clusters that are being frozen into their $\tilde{\sigma}^{x}=-1$ states by the transverse fields at this scale, and the spectral sum is now easily evaluated:

$$
\begin{aligned}
{\left[\chi_{\mathrm{loc}}\right]_{\mathrm{av}}(\omega) } & =\frac{1}{L} \sum_{m} \tilde{\sum}_{c} \tilde{\mu}_{c}\left|\left\langle m\left|\tilde{\sigma}_{c}^{z}\right| 0\right\rangle\right|^{2} \delta\left(\omega-\widetilde{E}_{m}\right) \\
& \sim \frac{n\left(\Gamma_{\omega}\right)}{\omega} R_{0}\left(\Gamma_{\omega}\right) \bar{\mu}_{0}\left(\Gamma_{\omega}\right)
\end{aligned}
$$

where we used the fact that all $\tilde{\mu}_{c}$ spins that are active in an effective cluster $c$ contribute identically, and $\bar{\mu}_{0}(\Gamma)$ is the average magnetic moment of the clusters that are being eliminated at scale $\Gamma$. (Note that here, and in the following, we simply write $\Gamma_{\omega}$ instead of $\Gamma_{\omega / 2}$ to avoid clutter in our notation; since we are interested only in the leading behavior, the difference is not important for our purposes.)

At criticality $(\delta=0)$, we obtain

$$
\left[\chi_{\mathrm{loc}}\right]_{\mathrm{av}}(\omega) \sim \frac{1}{\omega|\ln \omega|^{3-\phi}},
$$

for $\omega \ll \Omega_{0}$. For the average spin autocorrelation in imaginary time $\tau \gg \Omega_{0}^{-1}$ we then find 


$$
\left[C_{\mathrm{loc}}\right]_{\mathrm{av}}(\tau) \sim \frac{1}{|\ln \tau|^{2-\phi}}
$$

In the disordered phase $(\delta>0)$, we obtain

$$
\begin{gathered}
{\left[\chi_{\mathrm{loc}}\right]_{\mathrm{av}}(\omega) \sim \delta^{4-\phi} \frac{|\ln \omega|}{\omega^{1-1 / z(\delta)}},} \\
{\left[C_{\mathrm{loc}}\right]_{\mathrm{av}}(\tau) \sim \delta^{4-\phi} \frac{|\ln \tau|}{\tau^{1 / z(\delta)}},}
\end{gathered}
$$

for $\omega \ll \Omega_{\delta}$ and $\tau \gg \Omega_{\delta}^{-1}$. Here, we have used scaling solutions for the off-critical flows to write the answer for the local susceptibility and have chosen to express the powerlaw in terms of the dynamical exponent $z(\delta)$. From the scaling solution to the RG flow equations, we have $z^{-1}=2|\delta|$; this is to be thought of as the leading term in a small- $\delta$ expansion for $z^{-1}$. Written in terms of $z(\delta)$, our result Eq. (67) is valid more generally, and can be understood directly in the simple picture of the disordered phase given in F Sec. IVB: The average spin autocorrelation at large time $\tau$ is dominated by the (rare) spins that belong to the rare strongly coupled clusters (Griffiths regions) with low effective "flipping rates" (i.e., effective transverse fields) smaller than $\omega$ $\sim 1 / \tau$. The density of such clusters, which is also the density of the most numerous excitations at these low energies, is $n(\omega) \sim \omega^{1 / z}$ (this is fixed by the relationship $\tau \sim l^{z}$ between length and time scales, which serves as the definition of $z$ ). Most of these clusters have their effective flipping rates between $\omega$ and some fraction of $\omega$, and therefore effective moments of order $|\ln \omega|$ (since all clusters that are being eliminated at a fixed energy-scale $\Omega$ have roughly the same magnetic moment proportional to $|\ln \Omega|)$. Estimating the contribution of such Griffiths regions clearly gives us Eq. (67) including the factor of $|\ln \tau|$.

Finally, in the ordered phase $(\delta<0)$, we obtain

$$
\begin{gathered}
{\left[\chi_{\mathrm{loc}}\right]_{\mathrm{av}}(\omega) \sim|\delta|^{4-\phi} \frac{|\ln \omega|}{\omega^{1-2 / z(\delta)}},} \\
{\left[C_{\mathrm{loc}}\right]_{\mathrm{av}}(\tau) \sim|\delta|^{4-\phi} \frac{|\ln \tau|}{\tau^{2 / z(\delta)}},}
\end{gathered}
$$

for $\omega \ll \Omega_{\delta}$ and $\tau \gg \Omega_{\delta}^{-1}$. In contrast to the case of the disordered phase, the interpretation of Eq. (68) in terms of the picture of the ordered phase presented in F Sec. IVA is more subtle. In the ordered phase, the typical excitations at low energies $\omega \ll \Omega_{\delta}$ are classical-they are "domain walls" that "break" large clusters apart in the places where the clusters are held together by weak (effective) bonds of strength of order $\omega$. Such weak effective bonds represent the rare, large regions (Griffiths regions) that are locally in the disordered phase. These domain-wall excitations are the most numerous excitations that define the relationship between the energy and the length scales and determine the dynamical exponent $z(\delta)$. Such excitations, however, even if they are localized in the neighborhood of site $j$, do not con- tribute to $\chi_{j j}^{z z}(\omega)$ since they cannot be "excited" from the (classical) ground state by the action of $\sigma_{j}^{z}$. Excitations that do contribute involve much more rare ferromagnetic clusters that are flipping back and forth in isolation, with flipping rates of order $\omega \sim 1 / \tau$ or slower (of course, we exclude the macroscopic-cluster flipping at a rate of order $e^{-c L}$ as we are subtracting out the time-independent part of the autocorrelation). In the RG language, these are precisely the clusters that are decimated at energy scales of order $\omega$, i.e., that happen to have (at these scales) anomalously strong transverse fields of order $\omega$ (remember that we are in the ordered phase). A simple construction, however, clearly shows that the density of such regions is indeed $\sim \omega^{2 / z}$, as predicted by the scaling solution: For such a cluster to occur we need a ferromagnetic segment of length $\sim|\ln \omega|$ (which is not rare in the ordered phase) that is isolated (from eventually becoming a part of the macroscopic cluster) on each side by a disordered region of comparable length. Each of the two disordered regions is actually a "typical" Griffiths region at these energy scales, and the two are required to occur much closer together than their typical separation $\omega^{-1 / z}$; this explains the appearance of the power $2 / z$ in Eq. (68). The factor $|\ln \tau|$ again comes from the typical magnetic moments of such ferromagnetic droplets.

\section{Average local-energy autocorrelation $\left[C_{\mathrm{loc}}^{e}\right]_{\mathrm{av}}(\tau)$}

We begin by working out the leading-order renormalizations of the $\sigma^{x}$ operators: When a given spin $j$ is combined with another spin $k$ into a new cluster (i.e., when the strong bond $J_{j k}$ is being eliminated) the operator $\sigma_{j}^{x}$ renormalizes to $\left(\widetilde{h}_{(j k)} / h_{j}\right) \tilde{\sigma}_{(j k)}^{x}$, where $h_{j}$ is the transverse field on the spin $j$ before the decimation, $\widetilde{h}_{(j k)}$ is the effective transverse field on the new cluster $(j k)$, and $\tilde{\sigma}_{(j k)}^{x}$ is the effective "spin-flip" operator of this cluster (this rule ignores a constant term proportional to the identity operator, which is unimportant for our purposes as we are not interested in the timeindependent constant piece of the energy autocorrelation function). On the other hand, when the spin $j$ is eliminated, the operator $\sigma_{j}^{x}$ becomes effectively zero to first order in the nearby interactions (we again ignore any constants). Iterating this, the operator $\sigma_{j}^{x}$ is renormalized to $\left(\tilde{h}_{c} / h_{j}^{(0)}\right) \tilde{\sigma}_{c}^{x}$ if the spin $j$ is active in some cluster $c$ with the effective field $\widetilde{h}_{c}$, and is renormalized to zero if the spin is not active; here $h_{j}^{(0)}$ is the original (bare) transverse field on the spin $j$.

We now run the RG down to energy scale $\Omega_{\text {final }}=\omega / 2$ and rewrite the spectral sum as

$$
\left[\chi_{\mathrm{loc}}^{e}\right]_{\mathrm{av}}(\omega)=\frac{1}{L} \sum_{m}^{\sim} \tilde{\sum}_{c} \tilde{g}_{c}\left|\left\langle m\left|\tilde{\sigma}_{c}^{x}\right| 0\right\rangle\right|^{2} \delta\left(\omega-\widetilde{E}_{m}\right),
$$

where

$$
\tilde{g}_{c}=\sum_{j \in c}\left(\frac{\tilde{h}_{c}}{h_{j}^{(0)}}\right)^{2} .
$$

In the last equation $\Sigma_{j \in c}$ is over all spins that are active in a given cluster $c$. Note that at low energies the effective 
cluster-field $\widetilde{h}_{c}$ is only weakly correlated with each of the bare fields $h_{j}^{(0)}$; if the bare-field distribution is not too broad, we can approximate $h_{j}^{(0)} \sim \Omega_{0}$, and write $\tilde{g}_{c} \sim \tilde{\mu}_{c}\left(\tilde{h}_{c} / \Omega_{0}\right)^{2}$, where $\tilde{\mu}_{c}$ is the moment of the cluster $c$. Doing this clearly misses some nonuniversal numerical factor of order one that depends on the bare (high-energy) physics. This factor is, in principle, a random quantity that differs from one cluster to another; however, this number is expected to be roughly the same for all clusters that contribute to the spectral sum at low frequencies due to averaging, since such clusters are all large, and in some sense similar. Thus, we expect that the low-frequency behavior is not affected. (Note that we would have been spared this discussion if we were to analyze the spectral sum with matrix elements of $h_{j}^{(0)} \sigma_{j}^{x}$, which is anyway a more natural operator to consider when thinking of the local energy fluctuations.) The excitations that contribute to the spectral sum Eq. (69) correspond to transitions from the $\tilde{\sigma}_{j}^{z}=\tilde{\sigma}_{k}^{z}$ states to the $\tilde{\sigma}_{j}^{z}=-\tilde{\sigma}_{k}^{z}$ states of two (effective) spins $j$ and $k$ that are being combined into one cluster $\tilde{\sigma}_{(j k)}$ by a strong bond at the energy-scale $\Omega_{\text {final }}$. Since the log-field distribution is broad (we are near the critical point and at low energy scales), for such a pair of spins to contribute significantly the transverse field on at least one of the two spins involved must be of order $\omega$; thus, we have

$$
\left[\chi_{\mathrm{loc}}^{e}\right]_{\mathrm{av}}(\omega) \sim \frac{\omega}{\Omega_{0}^{2}} n\left(\Gamma_{\omega}\right) P_{0}\left(\Gamma_{\omega}\right) R_{0}\left(\Gamma_{\omega}\right) \bar{\mu}_{0}\left(\Gamma_{\omega}\right),
$$

from which we immediately read-off our results.

At criticality, for $\omega \ll \Omega_{0}$ and $\tau \gg \Omega_{0}^{-1}$, we obtain

$$
\begin{gathered}
{\left[\chi_{\mathrm{loc}}^{e}\right]_{\mathrm{av}}(\omega) \sim \frac{\omega}{|\ln \omega|^{4-\phi}},} \\
{\left[C_{\mathrm{loc}}^{e}\right]_{\mathrm{av}}(\tau) \sim \frac{1}{\tau^{2}|\ln \tau|^{4-\phi}} .}
\end{gathered}
$$

Away from the critical point, both in the disordered phase and in the ordered phase, we obtain

$$
\begin{gathered}
{\left[\chi_{\text {loc }}^{e}\right]_{\mathrm{av}}(\omega) \sim|\delta|^{5-\phi} \omega^{1+2 / z(\delta)}|\ln \omega|,} \\
{\left[C_{\mathrm{loc}}^{e}\right]_{\mathrm{av}}(\tau) \sim|\delta|^{5-\phi} \frac{|\ln \tau|}{\tau^{2+2 / z(\delta)}},}
\end{gathered}
$$

for $\omega \ll \Omega_{\delta}$ and $\tau \gg \Omega_{\delta}^{-1}$; in the last formula we again used $z(\delta)$ as a more physical parameter characterizing the Griffiths phase at a given $\delta$. The off-critical energy autocorrelation function thus behaves similarly in the two phases, as expected from duality. It is again possible to interpret these results in terms of the statistical properties of appropriate rare regions that dominate the average energy autocorrelation at long times. As the results (and their interpretation) are identical in either phase, we sketch only the interpretation on the ordered side: As we have already noted, for a region to have significant energy fluctuations at the frequency scale of order $\omega$, it must contain two adjoining segments both having a characteristic energy of order $\omega-$ a predominantly disordered segment (in the RG language, an effective bond with $\widetilde{J} \sim \omega$ across this segment) and a predominantly ordered segment (in the RG language, a cluster with $\tilde{h} \sim \omega$ ). Clearly, the predominantly ordered segment with effective transverse field $\sim \omega$ can exist only if it is also isolated on the other side from the rest of the system by another predominantly disordered segment having the same characteristic energy scale. This situation has already been analyzed in the context of the spin autocorrelations in the ordered phase, and clearly one recovers precisely Eq. (73) from such an analysis.

\section{Autocorrelations of the boundary spin}

So far, we have calculated average autocorrelations for the spins in the bulk. Calculations for the first spin $\sigma_{1}$ in a semi-infinite chain $j \geqslant 1$ proceed analogously (using the detailed characterization of the boundary spin from F Sec. V), and we will simply state the results.

For the spin autocorrelation we find, at criticality,

$$
\left[C_{1}\right]_{\mathrm{av}}(\tau) \sim \frac{1}{|\ln \tau|},
$$

while away from the critical point

$$
\left[C_{1}\right]_{\mathrm{av}}(\tau) \sim \frac{\delta^{2}}{\tau^{1 / z(\delta)}} .
$$

As in the bulk case, we can interpret the average off-critical spin autocorrelation Eq. (75) in terms of the rare instances that dominate this average. In this case, the corresponding rare regions must start at $\sigma_{1}$ - this explains the absence of a $|\ln \tau|$ factor in Eq. (75) compared to the bulk results Eqs. (67) and (68). The only other difference is that in the ordered phase we do not need to isolate the ferromagnetic droplet (containing $\sigma_{1}$ ) from the left.

For the energy autocorrelation we find, at criticality,

$$
\left[C_{1}^{e}\right]_{\mathrm{av}}(\tau) \sim \frac{1}{\tau^{2}|\ln \tau|^{3}},
$$

while in the disordered phase

$$
\left[C_{1}^{e}\right]_{\mathrm{av}}(\tau) \sim \frac{\delta^{3}}{\tau^{2+2 / z(\delta)}},
$$

and in the ordered phase

$$
\left[C_{1}^{e}\right]_{\mathrm{av}}(\tau) \sim \frac{|\delta|^{3}}{\tau^{2+1 / z(\delta)}} .
$$

The average off-critical energy autocorrelation of the boundary spin differs from that of the bulk spins in exactly the same way (and for the same reasons) as in the case of the spin autocorrelation.

\section{Comparison with numerics}

The first paper of Ref. 17 computed average spin autocorrelations (in both phases for the boundary spin, but only in 
the disordered phase for bulk spins). For the boundary spin, the fits for $1 / z$ using the scaling form identical to our Eq. (75) in both ordered and disordered phases were in good agreement with other measures of $1 / z$. For bulk spins, similar fits produced values of $1 / z$ consistently smaller than obtained by other means. This could be explained by the logarithmic factor, predicted by our Eq. (67), which would cause a power law fit to underestimate $1 / z$. We are not aware of numerical data for the bulk-spin autocorrelation in the ordered phase, where we predict a scaling form with a power of $2 / z$, Eq. (68), different from the naive expectation of $1 / z$.

The second paper of Ref. 17 computed the average bulkenergy autocorrelation in the disordered phase, fitting to $G_{\text {loc }}^{e} \sim 1 / \tau^{2+1 / z}$. We predict instead Eq. (73), with a different power $1 / \tau^{2+2 / z}$ and an additional $\ln \tau$ factor, due to the properties of the Griffiths regions that dominate here. We suggest that the numerical evidence in that paper is probably affected by the finite-size effects and the missing logarithmic factor, which always tends to underestimate the "apparent" exponents of the fitted power laws. We also predict a different power for the end-spin-energy autocorrelation in the disordered phase, Eq. (77). For the end-spin case, the fitting should be more straightforward, since there are no additional logarithmic factors. It is hoped that future numerical work will reexamine this question in view of our new results.

\section{Dynamic structure factor of the spins}

Let us now briefly consider the dynamic structure factor $S^{z z}(k, \omega)$ defined as

$$
S^{z z}(k, \omega)=\frac{1}{L} \sum_{m}\left|\left\langle m\left|\sum_{j} e^{i k x_{j}} \sigma_{j}^{z}\right| 0\right\rangle\right|^{2} \delta\left(\omega-E_{m}\right) .
$$

$S^{z z}(k, \omega)$ characterizes the spatial structure of the excitations at energy $\omega$. Proceeding as before, we find

$$
S^{z z}(k, \omega) \sim \frac{n\left(\Gamma_{\omega}\right)}{\omega} R_{0}\left(\Gamma_{\omega}\right) \overline{\left|\mu_{0}(k)\right|^{2}}\left(\Gamma_{\omega}\right),
$$

where $\overline{\left|\mu_{0}(k)\right|^{2}}(\Gamma)$ is the average modulus squared of the effective magnetic moment at wave vector $k$ for the clusters that are being eliminated at scale $\Gamma$; for a given cluster $c$, this effective moment is defined as $\mu_{c}(k)=\Sigma_{j \in c} e^{i k x_{j}}$. The dynamic structure factor can also be written in terms of the function $D\left(\beta, x \mid \Gamma_{\omega}\right)$ defined in F Sec. III B 4; we have

$$
S^{z z}(k, \omega) \sim \frac{\hat{D}\left(0, k \mid \Gamma_{\omega}\right)}{\omega},
$$

where $\hat{D}\left(0, k \mid \Gamma_{\omega}\right)$ is the Fourier transform of $D\left(0, x \mid \Gamma_{\omega}\right)$ at wave vector $k$. We have not attempted to analyze $D\left(\beta, x \mid \Gamma_{\omega}\right)$, even though a detailed characterization is likely to be possible (see F Sec. III B 4). Instead, we will only analyze the behavior of the dynamic structure factor in some limiting cases using the scaling picture.

First, consider the system at criticality. Fix wave vector $k$. Then, for $\Gamma \ll \Gamma_{k} \equiv 1 / \sqrt{k}$ the effective cluster moments at wave vector $k$ "add coherently" (more precisely, the real parts of the effective moments of the clusters that are being combined into bigger clusters are of the same sign) and therefore scale as $\Gamma^{\phi}$. At scales $\Gamma \gg \Gamma_{k}$, the effective moments at $k$ "add incoherently" (the real parts of the moments being combined can be of any relative sign) and therefore

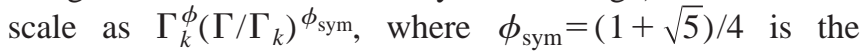
growth exponent for the cluster moments distributed symmetrically around zero (see Appendix of Ref. 4). Thus, we arrive at the following scaling form for the dynamic structure factor at criticality

$$
S^{z z}(k, \omega) \sim \frac{\Gamma_{\omega}^{2 \phi}}{\omega \Gamma_{\omega}^{3}} \Phi\left(k \Gamma_{\omega}^{2}\right),
$$

where $\Phi(x) \sim$ const for $x \ll 1$ and $\Phi(x) \sim 1 / x^{\phi-\phi_{\text {sym }}}$ for $x$ $\gg 1$. We cannot, however, address the regime $k \Gamma_{\omega}^{2} \sim 1$ by such a scaling analysis.

Now, consider the system that is not critical, either in the disordered or in the ordered phase, in the regime $\Gamma_{\omega} \gg \Gamma_{\delta}$. The length and the magnetic moment of a cluster that is eliminated at scale $\Gamma \gg \Gamma_{\delta}$ are sharply defined: $l_{0}(\Gamma)$ $=c_{l}\left(\Gamma / \Gamma_{\delta}\right) \Gamma_{\delta}^{2}+O\left(\sqrt{\Gamma / \Gamma_{\delta}} \Gamma_{\delta}^{2}\right)$ and $\mu_{0}(k=0, \Gamma)=c_{\mu}\left(\Gamma / \Gamma_{\delta}\right) \Gamma_{\delta}^{\phi}$ $+O\left(\sqrt{\Gamma / \Gamma_{\delta}} \Gamma_{\delta}^{\phi}\right)$, where $c_{l}$ and $c_{\mu}$ are numerical constants of order one. Such a cluster has some internal structure on the length scales below the correlation length $\sim \delta^{-2}$, but "looks" fairly uniform on larger length scales. Then, for the wave vectors $k \ll \delta^{2}$ we have

$$
\overline{\left|\mu_{0}(k)\right|^{2}}(\Gamma) \sim \frac{\delta^{4-2 \phi}}{k^{2}}\left[1+\cos \left(c_{l} k \Gamma /|\delta|\right) e^{-c k^{2} \Gamma /|\delta|^{3}}\right] ;
$$

note the oscillatory behavior at $k \sim|\delta| / \Gamma$ due to the "sharpness" of the lengths $l_{0}$ of the clusters; the gradual suppression of this oscillatory behavior at larger wave vectors comes from the uncertainty in $l_{0}$, which is much smaller than $l_{0}$ itself. To obtain the dynamic structure factor $S^{z z}(k, \omega)$ we simply need to multiply this "cluster structure factor" by the density $\rho(\omega)$ of such clusters at energy $\omega: \quad \rho(\omega)$ $\sim \delta^{3} / \omega^{1-1 / z(\delta)}$ in the disordered phase and $\rho(\omega)$ $\sim \delta^{3} / \omega^{1-2 / z(\delta)}$ in the ordered phase.

\section{A DISCUSSION OF $T \neq 0$ PROPERTIES}

So far we have calculated various dynamical and transport quantities at $T=0$. These results are clearly valid even at $T$ $\neq 0$ so long as the probe frequency $\omega$ satisfies $T \lesssim \omega$. Unfortunately, it is not straightforward to generalize our calculations to the complementary low-frequency regime $(\omega \ll T)$ dominated by thermal effects. There is, however, one exception. As mentioned earlier, the spin- $1 / 2 X X$ chain is equivalent to a model of spinless fermions with random nearestneighbor hopping and zero chemical potential. It should come as no surprise that the free-fermion nature of this problem allows us to straightforwardly calculate some dynamical and transport properties at small nonzero temperature. We begin this section by formulating a fermion analog of the RG procedure used for the spin chains. We will then use this RG approach to work out the low-frequency, low-temperature 
dynamical conductivity and the $z z$ component of the dynamic structure factor for the spin-1/2 $X X$ chain without any restriction on the relative magnitudes of $\omega$ and $T$ (the calculation of the perpendicular component of the structure factor for $\omega<T$ is much more complicated, and we will only be able to discuss its qualitative behavior). Naturally, these results are not at all generic, relying as they do on the freefermion nature of the problem. On the other hand, a weak $J^{z}$ coupling, which corresponds to the nearest-neighbor interaction between the fermions, is strongly irrelevant in the RG sense at the free-fermion point, and the system flows to the noninteracting point. Since this noninteracting limit is singular as far as finite-temperature transport properties are concerned, we have here an example of a "dangerously irrelevant operator," and the important physical question is how this weak, irrelevant interaction affects the $T \neq 0$ transport near the noninteracting point. ${ }^{32}$ This is what we turn to at the end of this section.

\section{A. Free-fermion $R G$}

The free-fermion problem $\mathcal{H}=\Sigma_{j} t_{j}\left(c_{j}^{\dagger} c_{j+1}+c_{j+1}^{\dagger} c_{j}\right)$ has been the subject of extensive investigation in the past using a variety of techniques (see, e.g., Ref. 25 and references therein). For our purposes, it is most convenient to introduce a RG procedure analogous to the singlet RG used in the spin problem. We formulate this procedure directly in terms of the corresponding single-particle Shrödinger problem $\mathbf{H}$ $=\sum_{j} t_{j}(|j\rangle\langle j+1|+| j+1\rangle\langle j|)$; this RG is, for the case of the Hamiltonian above, essentially just an efficient way of (approximately) diagonalizing random symmetric tridiagonal matrices with zeroes on the diagonal. We begin with the observation that the particle-hole symmetry of the problem causes eigenstates to occur in pairs, with energies $\pm \epsilon$. The strong-randomness RG proceeds by eliminating, at each step, such a pair of states with energies at the top and bottom of the band: One finds the largest (in absolute value) hopping amplitude in the system, say $t_{2}$ connecting sites 2 and 3 ; this defines the bandwidth $\Omega_{0}=2 \times \max \left\{\left|t_{j}\right|\right\}$ of the original problem. If the distribution of the $t_{j}$ is broad, the symmetric and antisymmetric wave functions living on these two sites will be good approximations to eigenstates with energies $\pm \Omega_{0} / 2$, as $t_{1 / 3}$ will typically be much smaller in magnitude than $t_{2}$. The couplings $t_{1 / 3}$ can then be treated perturbatively, and eliminating the high-energy states living on the sites 2 and 3 results in an effective hopping amplitude $\tilde{t}_{1}=-t_{1} t_{3} / t_{2}$ between the neighboring sites 1 and 4 . More precisely, in the effective Hamiltonian that describes the remaining $L-2$ states, the block 1-2-3-4 is represented as $\widetilde{\mathcal{H}}_{1-4}$ $=\tilde{t}_{1}(|\widetilde{1}\rangle\langle\widetilde{4}|+| \widetilde{4}\rangle\langle\widetilde{1}|)$, where the states $|\widetilde{1}\rangle$ and $|\widetilde{4}\rangle$ are essentially the original $|1\rangle$ and $|4\rangle$ states up to $O\left(t_{1 / 3} / t_{2}\right)$ corrections. This rule is essentially identical to the rule for the singlet $\mathrm{RG}$ at the $X X$ point, as the additional minus sign can be "gauged away" in the nearest-neighbor model in one dimension; we will, in fact, only keep track of the absolute values of the $t_{j}$. The distribution of $\left|t_{j}\right|$ in the renormalized problem with bandwidth $\Omega$ will thus be the same as the renormalized distribution of $J^{\perp}$ at cutoff scale $\Omega$ in the sin- glet RG for the spin problem. The analysis of the asymptotic validity of this approach thus carries over unchanged from the singlet RG.

This procedure can therefore be iterated to reach lower and lower energies; at each stage we trade in our current problem for a new problem defined on two fewer sites. This new problem will have the same low-energy eigenvalues as our original problem. However, evaluating matrix elements of operators between two low-energy states requires some care, as the states $|\tilde{j}\rangle$ in terms of which the renormalized problem is written are different from the states $|j\rangle$ of the original problem. As in the singlet RG, this is best handled by renormalizing the operators as we go along, so that the matrix elements of the renormalized operators between the states of the new problem are the same as the matrix elements of the bare operators between the corresponding states of the original problem. This allows us to calculate various dynamical properties by evaluating the corresponding spectral sums exactly as in the spin language. At $T=0$, this amounts to nothing more than a restatement in terms of the fermions of our previous calculations. The new language, however, has one important advantage: thermal effects are easily incorporated into this framework, essentially because the noninteracting nature of the problem is made explicit. [We emphasize again that the RG finds all eigenstates of the free-fermion problem. The corresponding statement can also be made in terms of the singlet RG on the $X X$ spin chain: when eliminating a pair of spins 2 and 3 the effective Hamiltonians in all sectors (corresponding to the states $\left|s_{0}\right\rangle,\left|t_{0}\right\rangle$, and $\left|t_{ \pm 1}\right\rangle$, of the pair) are identical up to a sign of $\widetilde{J}_{14}^{\perp}$ in the $\left|t_{ \pm 1}\right\rangle$ sector.]

Finally, we note in passing that this RG procedure can be generalized to analyze other particle-hole symmetric freefermion problems in one and two dimensions (which are not immediately equivalent to any quantum-spin problem) as well as analyze the general properties of the Bogoliubov-de Gennes equation for quasiparticles in a one-dimensional superconducting wire in the absence of spin-rotation symmetry (the results of such an analysis will be published separately).

\section{B. $T \neq 0$ dynamics and transport at the $X X$ point}

Let us begin by working out the full $T$ and $\omega$ dependence of the dynamical conductivity, Eq. (55), at the free fermion point. Our first task is to work out the rules that govern the renormalization of the current operators $\mathbf{T}(j)$. Assume once again that the hopping element $t_{2}$ has maximum magnitude. We wish to work out what operators we should use in place of $\mathbf{T}(1), \mathbf{T}(2)$, and $\mathbf{T}(3)$ when we renormalize down to lower energies by eliminating the corresponding two states at the top and bottom of the band (the other current operators to the left and right of this segment will be unchanged to leading order by this elimination). An explicit perturbative calculation immediately yields $\widetilde{\mathbf{T}}(2)=\widetilde{\mathbf{T}}(1 / 3)$ $=i \widetilde{t}_{1}(|\widetilde{1}\rangle\langle\widetilde{4}|-| \widetilde{4}\rangle\langle\widetilde{1}|)$; this is completely analogous to the rule obtained in the spin representation, and as before, we will call this operator $\widetilde{\mathbf{T}}(1)$ for consistency of notation. 


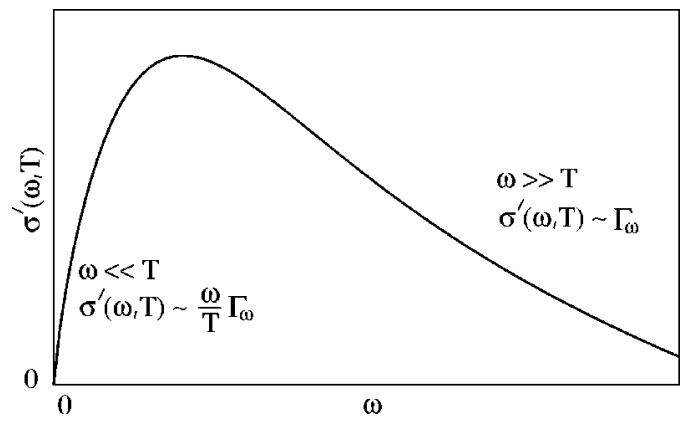

FIG. 7. A plot of the frequency dependence of $\sigma^{\prime}(\omega)$ at low $T \neq 0$ at the $X X$ point. Note that this result is expected to break down below a frequency scale $1 / \tau_{t r} \ll T$ when a small but nonzero $J^{z}$ interaction is turned on (see Sec. VI C).

As we carry out the RG and reduce the bandwidth by removing states from the top and bottom of the band, the above result implies that $\Sigma_{j} \mathbf{T}(j)$ renormalizes to $\widetilde{\Sigma}_{j} \widetilde{l}_{j} \widetilde{T}(j)$, where $j$ now labels the sites of the renormalized problem with bandwidth $\Omega$, and the $\widetilde{l}_{j}$ are the lengths of the renormalized bonds in this problem. With this in hand, we run the RG until the bandwidth is reduced to $\Omega_{\text {final }}=\omega$ and rewrite the spectral sum Eq. (55) as

$$
\begin{aligned}
\sigma^{\prime}(\omega)= & \frac{1}{\omega L} \sum_{\mu_{1}, \mu_{2}}^{\sim} \mid\left\langle\widetilde{\phi}_{\mu_{2}}\left|\sum_{j}^{\sim} \widetilde{l}_{j} \widetilde{\mathbf{T}}(j)\right| \widetilde{\phi}_{\mu_{1}}||^{2}\right. \\
& \times\left[f\left(\epsilon_{\mu_{1}}\right)-f\left(\epsilon_{\mu_{2}}\right)\right] \delta\left(\omega-\epsilon_{\mu_{2}}+\epsilon_{\mu_{1}}\right) .
\end{aligned}
$$

Because of the extremely broad distribution of the $\widetilde{t}_{j}$, the dominant contribution to the sum Eq. (84) comes from transitions between the two members, one at the bottom and the other at the top of the renormalized band, of each pair of states that is being eliminated at this energy scale. The matrix element for this transition is just $\widetilde{l}|\omega| / 2$, where $\widetilde{l}$ is the length of the hop in question. In the thermodynamic limit, we thus have

$$
\begin{aligned}
\sigma^{\prime}(\omega)= & {[f(-\omega / 2)-f(\omega / 2)] } \\
& \times \frac{n\left(\Gamma_{\omega}\right)}{\omega} \int d l d \zeta \omega^{2} l^{2} P\left(\zeta, l \mid \Gamma_{\omega}\right) \delta\left(\omega-\omega e^{-\zeta}\right) \\
= & \frac{\sinh (\omega / 2 T)}{2 \cosh ^{2}(\omega / 4 T)} \sigma_{T=0}^{\prime}(\omega),
\end{aligned}
$$

which is the leading behavior for $\omega, T \ll \Omega_{0}$. This result smoothly interpolates between the logarithmic frequency dependence seen earlier for $\omega \gg T$ and the limiting form $\sigma^{\prime}(\omega) \sim \omega \ln \left(\Omega_{0} / \omega\right) / T$ valid for $\omega \ll T$-a plot of this frequency dependence is shown in Fig. 7.

Let us now turn to the $T \neq 0$ spin dynamic structure factor at low frequencies in the vicinity of $k=\pi / a$. In the singleparticle language, the spectral representation for $S^{z z}(k, \omega)$ reads

$$
\begin{aligned}
S^{z z}(k, \omega)= & \frac{1}{\left(1-e^{-\omega / T}\right) L} \sum_{\mu_{1}, \mu_{2}}\left|\left\langle\phi_{\mu_{2}}\left|\hat{\mathbf{S}}^{z}(k)\right| \phi_{\mu_{1}}\right\rangle\right|^{2} \\
& \times\left[f\left(\epsilon_{\mu_{1}}\right)-f\left(\epsilon_{\mu_{2}}\right)\right] \delta\left(\omega-\epsilon_{\mu_{2}}+\epsilon_{\mu_{1}}\right) .
\end{aligned}
$$

Here $\hat{\mathbf{S}}^{z}(k) \equiv \sum_{j} \mathbf{S}^{z}(j) e^{i k x_{j}}$ is the Fourier transform of the position-dependent matrix operator $\mathbf{S}^{z}(j)$; in the real-space basis $\mathbf{S}^{z}(j)=\mathbf{n}(j)-1 / 2$, where $\mathbf{n}(j)=|j\rangle\langle j|$. This spectral sum can also be evaluated within our RG approach. The leading-order operator renormalizations in this case are, in complete analogy with the spin problem, very simple: each $\mathbf{S}^{z}(j)$ remains unchanged unless a state living on site $j$ is eliminated, in which case $\mathbf{S}^{z}(j)$ renormalizes to a multiple of the identity. As before, we run the RG till the bandwidth is reduced to $\Omega_{\text {final }}=\omega$ and do the spectral sum with the renormalized operators in the new problem. This renormalized sum may be evaluated by again recognizing that it is dominated by transitions between pairs of states with energies $\pm \omega / 2$ that live on pairs of sites connected by "strong" hopping amplitudes (of magnitude $\omega / 2$ ) in the renormalized problem. The corresponding matrix element is just (1 $\left.-e^{i k \tau}\right) / 2$, where $\tilde{l}$ is the length of the hop in question. Counting the contributions exactly as in our zerotemperature calculations, we thus get

$$
S^{z z}(k, \omega)=\frac{1}{\left(1+e^{-\omega / 2 T}\right)^{2}} S_{T=0}^{z z}(k, \omega) .
$$

Thus, we see that $S^{z z}$ is essentially unaffected by thermal fluctuations at the $X X$ point; in particular, the low-frequency divergence is not cut off by temperature effects even when $\omega \ll T$.

A similar analysis can clearly be performed in the $X X$-RD phase. Again, both $\sigma^{\prime}(\omega)$ and $S^{z z}(k, \omega)$ at $T>0$ are simply given by the corresponding expressions at $T=0$ multiplied by simple functions of $\omega / T$, exactly as in Eqs. (85) and (87). Thus, though temperature effects are simple to work out at the $X X$ point and in the $X X$-RD phase, the results are rather special due to the free-fermion character of the problem.

\section{Going beyond the free-fermion results}

What happens when we turn on the nearest-neighbor interaction? This is the question we need to address next.

Let us first consider the effects of small $J^{z}$ couplings added on to the $X X$ model. The analysis of Ref. 4 shows that this term is irrelevant in the RG sense; the typical value of $\widetilde{J}^{z} / \widetilde{J}^{\perp}$ at log-cutoff $\Gamma$ scales as $\left(\widetilde{J}^{z} / \widetilde{J}^{\perp}\right) \sim u_{0} \exp \left(-c \Gamma^{\phi}\right)$, where $c$ is an $O(1)$ constant, $\phi$ is the golden mean (1 $+\sqrt{5}) / 2$, and $u_{0}$ is the typical value of $J^{z} / J^{\perp}$ in the microscopic model. A useful way of thinking about the lowfrequency behavior of the conductivity is as follows: Imagine running the RG till the cutoff $\Omega \sim T$. In this renormalized problem the typical $\left(\widetilde{J}^{z} / \widetilde{J}^{\perp}\right) \sim u_{0} \exp \left(-c \Gamma_{T}^{\phi}\right)$, where $\Gamma_{T}$ $\equiv \ln \left(\Omega_{0} / T\right)$. In the fermion language, this is the typical value of the ratio of the nearest-neighbor interactions to the hopping amplitudes. In this renormalized problem, a naive Fermi's golden rule estimate of the corresponding inelastic col- 
lision rate due to interactions gives $1 / \tau_{\text {coll }}$ $\sim u_{0}^{2} T \exp \left(-2 c \Gamma_{T}^{\phi}\right)$. This gives us a frequency scale below which our $T \neq 0$ free-fermion results are expected to break down as a result of the residual interaction effects.

Unfortunately, we are unable to do a controlled calculation that determines the transport properties in the frequency regime $\omega<1 / \tau_{\text {coll }}$. The best we can do is to work out what a naive scaling argument would predict for the dc limit of the conductivity. The basic idea is as follows: The collision rate may be converted into a corresponding dephasing length $L_{\text {coll }}$ by appealing to the activated scaling that is a characteristic of our problem. This gives $L_{\text {coll }} \sim \ln ^{2}\left(\tau_{\text {coll }}\right) \sim \Gamma_{T}^{2 \phi}$. This is the length scale beyond which quantum coherence is lost due to inelastic collisions. Now, we can imagine breaking up the system into blocks of length $L_{\text {coll }}$. A dc current $I$ passing through the system will see a chain of resistors corresponding to these blocks; the resistance values of each of these blocks is simply given by the $T=0$ Landauer resistance of the corresponding system of length $L_{\text {coll }}$. The voltage developed across a system of total length $L$ will therefore be $V$ $=I \sum_{j=1} L / L_{\mathrm{coll}} R_{j}=I L R_{\mathrm{av}}\left(L_{\mathrm{coll}}\right) / L_{\mathrm{coll}}$. Since $R_{\mathrm{av}}\left(L_{\mathrm{coll}}\right)$ $\sim e^{c_{1} L_{\text {coll }}}$ [where $c_{1}$ is an $O(1)$ scale factor], the dc conductivity works out to be $\sigma_{\mathrm{dc}} \sim L_{\text {coll }} e^{-c_{1} L_{\text {coll }}}$ $\sim \ln ^{2 \phi}\left(\Omega_{0} / T\right) e^{-c^{\prime} \ln ^{2 \phi}\left(\Omega_{0} / T\right)}$. Note that in the absence of interactions, we had earlier found $\sigma^{\prime}(\omega) \rightarrow 0$ as $\omega \rightarrow 0$ at $T>0$; our scaling argument implies that interactions render this conclusion invalid. Unfortunately, while this scaling argument is certainly plausible, the question of the true lowfrequency limit can only be settled by a controlled calculation in the regime $\omega \ll 1 / \tau_{\text {coll }}$, which is beyond our current capabilities.

The above arguments also suggest that $S^{z z}(k, \omega)$ will deviate from the $T \neq 0$ free-fermion result for $\omega<1 / \tau_{\text {coll }}$. In particular, one expects that the $\omega \rightarrow 0$ divergence of $S^{z z}$ would be cut off below this frequency scale. Similar behavior is also expected of $S^{+-}$, but again, what is really needed is a controlled calculation as opposed to a scaling argument. Note also that we expect something different at the $X X X$ and $X X Z C$ quantum critical points: since the theory at these critical points already includes interactions, one expects that $1 / \tau_{\text {coll }} \sim T$, and the relaxational behavior characteristic of an interacting system at finite temperature will set in for $\omega \sim T$, in contrast to the behavior in the vicinity of the $X X$ point.

\section{PROSPECTS FOR EXPERIMENTAL TESTS}

Previous experimental work on one-dimensional randomexchange Heisenberg antiferromagnetic spin chains has characterized the dynamics of these systems in terms of the observed NMR $1 / T_{1}$ relaxation rate, ${ }^{33}$ and ESR relaxation rates and linewidths. ${ }^{11}$

As far as the NMR measurements are concerned, our calculations are unfortunately not directly relevant to the experimental measurements of $1 / T_{1}$. This may be seen as follows: In the usual case of a pure, translationally invariant system, $1 / T_{1}$ is directly related, by Fermi's golden rule, to the local dynamic structure factor $S_{\text {loc }}$ evaluated at frequency $\omega$ equal to the nuclear-resonance frequency $\gamma_{N} H$, where $\gamma_{N}$ is the nuclear magnetic moment and $H$ is the external field. In a random system, with a broad variation in the value of $S_{\text {loc }}(\omega)$, the following question immediately arises: what measure of the distribution of $S_{\text {loc }}(\omega)$ does the experimentally measured $1 / T_{1}(H)$ reflect?

Now, we have seen that the average $S_{\text {loc }}(\omega)$ diverges strongly as $\omega \rightarrow 0$ at $T=0$. Naively, one might have thought that this would imply a corresponding divergence in $1 / T_{1}$ at small $H$, at least when $T \ll H$. However, the divergence in $S_{\text {loc }}(\omega)$ comes from a few very rare sites that give a very large contribution. Clearly, the observed $1 / T_{1}$ will be completely insensitive to this effect, since all that will happen is that a tiny fraction of nuclear spins (in the neighborhoods of those rare electron spins that have significant spin fluctuations at the frequency $\omega=\gamma_{N} H$ ) will flip almost instantaneously, while the rest of the nuclear spins will have an extremely small probability to flip, and this is what will be reflected in the spin relaxation experiments. In this sense, it is the typical value of $S_{\text {loc }}(\omega)$ that is more relevant for comparisons with NMR $1 / T_{1}$ data. A typical nuclear spin will in fact have essentially no spin fluctuations to couple to at frequency $\omega=\gamma_{N} H$, it can therefore relax only by paying an activation energy that is set by $\gamma_{e} H$ (where $\gamma_{e} \gg \gamma_{N}$ is the electron magnetic moment) since the external field acts to freeze out all modes below this energy scale in most of the system (with the exception of the rare regions alluded to above). The experiments actually do see activated behavior for $1 / T_{1}$ at finite temperature. However, the activation gap seems to scale as $\Delta \sim H^{1.6}$; the rough argument above of course cannot explain this non-trivial $H$ dependence of the observed activation energy.

Our second remark relates to the ESR linewidth measurements by Tippie and Clark. ${ }^{11}$ Here, again, our results do not address the experimentally relevant questions. This is because all our calculations for the $X X X$ case are done within the context of the simple Heisenberg exchange Hamiltonian, while the observed linewidth in the experiments is determined by other effects such as dipolar interactions or anisotropy.

Inelastic-neutron-scattering experiments, on the other hand, if they can be done on these systems, provide a direct testing ground for our predictions. We conclude with some remarks on the relevance of our calculations of the dynamic structure factor to such experiments. First of all, note that we considered randomness in the exchanges only, with the spins themselves assumed positioned on regular lattice sites; thus, our results are restricted to compounds with chemical disorder in exchanges. It is clear that small randomness in the positions of spins (e.g., due to thermal fluctuations) will result only in some further suppression (by the standard Debye-Waller factor at wave vector $k$ ) of the features relative to an overall background. In the dimer phase, a possible difference in the lengths of even and odd bonds will result only in some phase factor in the cosine of Eq. (28). Also note that the nonmagnetic neutron scattering from such spin chains will actually be suppressed near $k=\pi / a$, and this may facilitate a possible experimental observation of the predicted features. We caution, however, that while it would be extremely interesting to see the sharp oscillatory structure 
predicted in the Griffiths phases, this may be difficult to achieve without going to very low temperatures and energy transfers. Regarding transport, we hope that our results will motivate experiments to probe the spin conductivity in these systems.

\section{ACKNOWLEDGMENTS}

We thank P. W. Anderson, R. N. Bhatt, D. Dhar, D. S. Fisher, F. D. M Haldane, M. Hastings, A. Millis, A. Madhav, R. Moessner, S. Sachdev, T. Senthil, S. L. Sondhi, and A. Vishwanath for useful discussions. This work was supported by NSF Grant Nos. DMR-9809483 and DMR-9802468.

\section{APPENDIX: FINITE-SIZE SCALING FUNCTION FOR THE CONDUCTIVITY}

Consider a finite chain with an even number of sites $N_{I}$ $=L+1$, where $L$ is the length of the chain, and with free boundary conditions (a similar analysis can be carried out for chains with an odd number of sites, and also for chains with periodic boundary conditions). We want to calculate the real part of the dynamical conductivity averaged over the distribution of bond strengths in the limit of low frequencies and large $L$. We work this out for the $X X$ chain; the result in the presence of $J^{z}$ couplings will differ only in the values of some non-universal scale factors so long as the system does not develop Ising antiferromagnetic order in the thermodynamic limit. To proceed, we need to keep track of the joint distribution at scale $\Gamma$ of the number of remaining spins $N$, the $N-1$ couplings $\zeta_{i}$, and the corresponding bond lengths $l_{i}$. In a finite system, the couplings become correlated due to the constraint imposed by the finite length of the system. However, following Fisher and Young, ${ }^{34}$ we note that the couplings remain "quasi-independent," and can be described in terms of the infinite-chain distribution $P(\zeta, l \mid \Gamma)$ exactly as in Ref. 34. More precisely, if we also keep track of the lengths $l_{F}$ and $l_{R}$ of the "dead" regions (consisting of singlet pairs formed at higher energy-scales) at the left and right ends of the chain, then a distribution of the form

$$
\begin{aligned}
d \operatorname{Prob} & {\left[N ; \zeta_{1}, l_{1} \cdots \zeta_{N-1}, l_{N-1} ; l_{F}, l_{R} \mid L, \Gamma\right] } \\
= & a_{N}(L \mid \Gamma) P\left(\zeta_{1}, l_{1}\right) d \zeta_{1} \cdots P\left(\zeta_{N-1}, l_{N-1}\right) d \zeta_{N-1} \\
& \times \mathcal{L}\left(l_{F}\right) \mathcal{L}\left(l_{R}\right) \delta_{l_{1}+\cdots+l_{N-1}+l_{F}+l_{R}, L}
\end{aligned}
$$

for even $N \geqslant 2$ has its from preserved under renormalization if $a_{N}(L \mid \Gamma)$ is independent of $N$ with

$$
\frac{1}{a} \frac{d a}{d \Gamma}=2 P_{0}(\Gamma)=2 \int d l P(0, l) .
$$

Here, $P(\zeta, l \mid \Gamma)$ satisfies the same flow equation as in the infinite chain, and $\mathcal{L}(l \mid \Gamma)$ satisfies

$$
\frac{\partial \mathcal{L}}{\partial \Gamma}=\mathcal{L}(\cdot) *{ }_{l} P(0, \cdot) * \int_{0}^{\infty} P(\zeta, \cdot) d \zeta-P_{0} \mathcal{L} .
$$

In the above, the $\Gamma$ dependence is left implicit, and $f(\cdot) * l g(\cdot)$ is used to denote a (discrete) convolution in the length variables. For clarity, we work explicitly with discrete lengths, with $l_{F}$ and $l_{R}$ even, and $l_{i}$ odd integers; this is clearly preserved under the $\mathrm{RG}$.

We start the RG with $\Omega_{0}=1, \Gamma_{I}=0$, the initial bond distribution $P\left(\zeta \mid \Gamma_{I}\right)=e^{-\zeta}$ (this corresponds simply to choosing the initial $J^{\perp}$ to be uniformly distributed in the interval $[0,1]), l_{i}=1, l_{F}=l_{R}=0$, and $N_{I} \equiv L+1$; with initial distributions $P\left(\zeta, l \mid \Gamma_{I}\right)$ and $\mathcal{L}\left(l \mid \Gamma_{I}\right)$ normalized to unity, the normalization factor is $a\left(\Gamma_{I}\right)=1$. The dynamical conductivity is now given by

$$
\sigma^{\prime}(\omega, L)=\frac{1}{4} \frac{a\left(\Gamma_{\omega}\right)}{L} A\left(L \mid \Gamma_{\omega}\right)
$$

with $a(\Gamma)=(\Gamma+1)^{2}$ (for our specific choice of initial conditions), and

$$
\begin{aligned}
A(L \mid \Gamma)= & \sum_{N=2}^{L+1} '(N-1)_{l_{1}, l_{2}, \ldots, l_{N-1}, l_{F}, l_{R}} P\left(0, l_{1}\right) l_{1}^{2} \\
& \times \int_{0}^{\infty} P\left(\zeta_{2}, l_{2}\right) d \zeta_{2} \ldots \int_{0}^{\infty} P\left(\zeta_{N-1}, l_{N-1}\right) d \zeta_{N-1} \\
& \times \mathcal{L}\left(l_{F}\right) \mathcal{L}\left(l_{R}\right) \delta_{l_{1}+l_{2}+\cdots+l_{N-1}+l_{F}+l_{R}, L}
\end{aligned}
$$

where the sum is over even $N$.

Now, multiplying $A(L \mid \Gamma)$ by $e^{-y L}$ and summing over odd $L \geqslant 1$, i.e., doing a (discrete) Laplace transform in $L$, removes the constraint on the lengths, and we find

$$
A(y \mid \Gamma)=\mathcal{L}^{2}(y) Q(y) \frac{1+T^{2}(y)}{\left[1-T^{2}(y)\right]^{2}},
$$

where $Q(y)$ and $T(y)$ are respectively the Laplace transforms of $P(0, l) l^{2}$ and $\int_{0}^{\infty} P(\zeta, l) d \zeta$. Thus, we can straightforwardly work out $A(y)$, given $P(\zeta, y)$ and $\mathcal{L}(y)$. Using the results of Refs. 34 and 6, we can write the following explicit expressions for these two functions:

$$
\begin{gathered}
P(\zeta, y \mid \Gamma)=Y(y \mid \Gamma) e^{-\zeta u(y \mid \Gamma)}, \\
\mathcal{L}(y \mid \Gamma)=\frac{u(0 \mid \Gamma) u\left(y \mid \Gamma_{I}\right)}{u(y \mid \Gamma) u\left(0 \mid \Gamma_{I}\right)} \mathcal{L}\left(y \mid \Gamma_{I}\right),
\end{gathered}
$$

where $u(y \mid \Gamma)=D(y) \operatorname{coth}[D(y)(\Gamma+C(y))]$ and $Y(y \mid \Gamma)$ $=D(y) / \sinh [D(y)(\Gamma+C(y))]$. The functions $D(y)$ and $C(y)$ depend on the initial distribution $P\left(\zeta, y \mid \Gamma_{I}\right)$, and in our case are given by $D(y)=\sqrt{1-e^{-2 y}}$ and $D(y) C(y)=y$ $+\ln \left(1+\sqrt{1-e^{-2 y}}\right)$. Also, $\mathcal{L}\left(y \mid \Gamma_{I}\right)=1$.

With this in hand, it is a relatively simple matter to work out $A(L \mid \Gamma), L$ odd, by performing the inverse Laplace transform:

$$
A(L)=\frac{1}{\pi i} \int_{c-i \pi / 2}^{c+i \pi / 2} A(y) e^{y L} d y .
$$

In Sec. III D 5, we evaluated this integral numerically to compare the RG predictions with the results of the exactdiagonalization studies (note that in the main body of the paper we didn't make a distinction between $N_{I}$ and $L$, since 
it is irrelevant in the thermodynamic limit; in the more detailed notation of this appendix, our numerical results of Sec. III D 5 are for system sizes $N_{I}=128,256$, and 512).

In the scaling limit $\Gamma \gg 1, L \gg 1$, the integral Eq. (A9) for $A(L \mid \Gamma)$ is dominated by small $y$ and can be approximated by

$$
A(L \mid \Gamma)=2 \mathrm{LT}^{-1} A(y \mid \Gamma),
$$

where $\mathrm{LT}^{-1}$ denotes the inverse of the continuous Laplace transform. Moreover, in this limit, $A(y)$ may be worked out using the following scaling forms for $\mathcal{L}(y)$ and $P(\zeta, y)$ :

$$
\begin{gathered}
\mathcal{L}(y \mid \Gamma)=\frac{1}{\Gamma \sqrt{2 y} \operatorname{coth}[\Gamma \sqrt{2 y}]}, \\
P(\zeta, y \mid \Gamma)=\frac{\sqrt{2 y}}{\sinh [\Gamma \sqrt{2 y}]} e^{-\zeta \sqrt{2 y} \operatorname{coth}[\Gamma \sqrt{2 y}]} .
\end{gathered}
$$

Putting everything together, we can now write $A(L, \Gamma)$ $=\Gamma f\left(\Gamma^{2} / L\right)$, which immediately implies a scaling form for the conductivity: $\sigma^{\prime}(\omega, L)=\Gamma_{\omega} \Theta\left(\Gamma_{\omega}^{2} / L\right)$. Thus, we see that the dynamical conductivity in a finite system satisfies a scaling form that reflects the activated dynamical scaling at the
$X X$ fixed point. Note that while the scaling form holds more generally, the values of the nonuniversal scale factors that we have used are specific to our choice of initial distribution. Analyzing the behavior starting with an arbitrary initial distribution allows one to relate these nonuniversal scale factors to the properties of the initial distribution under the assumption that "bad decimations" early in the RG do not affect these values. Such an analysis allows us to write $\sigma^{\prime}(\omega, L)$ $=l_{v} \ln \left(\Omega_{0} / \omega\right) \Theta\left[l_{v} \ln ^{2}\left(\Omega_{0} / \omega\right) / L\right]$, with the microscopic energy-scale $\Omega_{0}$ and the microscopic length-scale $l_{v}$ precisely as defined in the main text. Moreover, it is clear that the same scaling function also describes the low-frequency dynamical conductivity in a large but finite system even in the presence of $J^{z}$ interactions as long as the system is in a random singlet state; only the values of the nonuniversal scale factors are expected to change.

While it is possible to calculate the full scaling-function $\Theta(x)$ by a detailed analysis of the inverse Laplace transform, we will confine ourselves here to working out $\Theta(x)$ in two limiting cases: For $x \ll 1, \Theta(x)=7 / 180$ (which correctly reproduces the infinite-size result, as it must), while in the limit $x \gg 1$ we have $\Theta(x)=e^{-x / 2} / \sqrt{2 \pi x}$. This is the result used in the Griffiths argument in Sec. III D.
${ }^{1}$ S. Sachdev, Philos. Trans. R. Soc. London, Ser. A 356, 173 (1998).

${ }^{2}$ D. Belitz and T. R. Kirkpatrick, Rev. Mod. Phys. 66, 261 (1994).

${ }^{3}$ Perspectives in Quantum Hall Effects: Novel Quantum Liquids in Low-Dimensional Semiconductor Structures, edited by S. D. Sarma and A. Pinczuk (Wiley, New York, 1997).

${ }^{4}$ D. S. Fisher, Phys. Rev. B 50, 3799 (1994).

${ }^{5}$ R. A. Hyman and Kun Yang, Phys. Rev. Lett. 78, 1783 (1997).

${ }^{6}$ D. S. Fisher, Phys. Rev. Lett. 69, 534 (1992); Phys. Rev. B 51, 6411 (1995).

${ }^{7}$ O. Motrunich, S. C. Mau, D. A. Huse, and D. S. Fisher, Phys. Rev. B 61, 1160 (2000).

${ }^{8}$ C. Monthus, O. Golinelli, and Th. Jolicoeur, Phys. Rev. Lett. 79, 3254 (1997).

${ }^{9}$ R. A. Hyman, Kun Yang, R. N. Bhatt, and S. M. Girvin, Phys. Rev. Lett. 76, 839 (1996).

${ }^{10}$ K. Damle, O. Motrunich, and D. A. Huse, Phys. Rev. Lett. 84, 3434 (2000).

${ }^{11}$ L. C. Tippie and W. G. Clark, Phys. Rev. B 23, 5846 (1981); 23, 5854 (1981).

${ }^{12}$ C. P. Landee et al., Bull. Am. Phys. Soc. 44, 1942 (1999).

${ }^{13}$ M. Takigawa et al., Phys. Rev. Lett. 76, 2173 (1996).

${ }^{14}$ C. Payen, E. Janod, K. Schoumacker, C. Batista, K. Hallberg, and A. Aligia, Phys. Rev. B 69, 2998 (2000).

${ }^{15}$ Kun Yang and R. A. Hyman, Phys. Rev. Lett. 84, 2044 (2000).

${ }^{16}$ Recent work (Ref. 7) has shown that the quantum Ising model in two dimensions can also be described by a strong-disorder effective theory at low energies. Low-frequency dynamical properties for the two-dimensional case can be readily obtained from these results using our approach; see also Ref. 35 for the calculation of average dynamical susceptibilities in the dilute quantum Ising system.
${ }^{17}$ F. Igloi et al., Phys. Rev. B 57, 11404 (1998); 59, 11308 (1999); A. P. Young, ibid. 56, 11691 (1997); J. Kisker and A. P. Young, ibid. 58, 14397 (1998).

${ }^{18}$ R. A. Hyman, Ph.D. dissertation, Indiana University, 1996.

${ }^{19}$ C. A. Doty and D. S. Fisher, Phys. Rev. B 45, 2167 (1992).

${ }^{20}$ C. Dasgupta and S. K. Ma, Phys. Rev. B 22, 1305 (1980).

${ }^{21}$ The fact that the volumes and moments of the Griffiths regions of a given characteristic energy are sharply defined can also be made plausible by reasoning as follows: These regions can be thought of as being selected by a saddle-point criterion that balances the probability of their occurrence against the strength of their contribution to, say, the susceptibility at a given low frequency. In the limit of very low frequencies, the corresponding saddle point becomes very sharp, leading to a sharp definition of the lengths and moments of these regions.

${ }^{22}$ While this paper was being written, we came across a preprint [F. Igloi et al., cond-mat/9911480 (unpublished)] that numerically calculates average autocorrelation functions at the $X X$ point and in the $X X$-RD phase and in addition obtains numerical information about the corresponding probability distributions. Their results for the averages provide a stringent numerical check of our analytical predictions, and the results are in complete agreement.

${ }^{23}$ We need to be critical about evaluating $\sigma^{\prime}(\omega)$ using the finitesize result Eq. (45). In the regime considered, the main contribution to the (average) dynamical conductivity at frequency $\omega$ of a finite segment of length $L$ comes from the cases when the last bond decimated has $\widetilde{J}^{\perp}=\omega$, i.e., precisely from the cases when the lowest gap of the segment is $\omega$; in almost all of these cases the bond stretches essentially across the whole length $L$ and the conductivity is simply related to the distribution of gaps in such segments of length $L$. Thus, two spins in a typical pair contributing to Eq. (45) (i.e., "resonating" at $\omega$ ) are near the 
two respective ends of such region and are likely to have strong $\widetilde{J}^{z}>\omega$ couplings with the rest of the system, so the pair will not contribute to the dynamical conductivity at all. Clearly, we need to consider cases when a pair with $\widetilde{J}^{\perp}=\omega$ occurs deep inside our $\mathrm{RS}$ region and is thus well-isolated from the rest of the system - in some sense, we need to consider distribution of the second energy-gap in such finite segments. One can analyze these situations along the lines of a similar analysis of Ref. 34 for the distribution of the first gap, with the result that the contributions to the dynamical conductivity from such events, which will give the actual $\sigma^{\prime}(\omega)$, are further suppressed as compared to Eq. (45) by an additional factor $\sim \exp \left(-c_{2}|\ln \omega|^{2} / L\right)$; this will modify the exponent $\alpha$ in Eq. (47) (which is unknown to us in any case from these arguments), but not the factor $|\ln \omega|^{2}$. Our scaling arguments in the main text provide a more consistent constructive approach for addressing these issues.

${ }^{24}$ T. P. Eggarter and R. Riedinger, Phys. Rev. B 18, 569 (1978).

${ }^{25}$ L. Balents and M. P. A. Fisher, Phys. Rev. B 56, 12970 (1997); H. Mathur, ibid. 56, 15794 (1997).

${ }^{26}$ There is also a calculation (Ref. 36) referred to in literature (Ref. 37) of the dynamical conductivity at the free-fermion point that relies on a diagrammatic resummation technique, which predicts that $\sigma^{\prime}(\omega) \rightarrow$ const as $\omega \rightarrow 0$, which is a weaker signature of the unusual localization properties of this problem than predicted by us. We have been unable to pinpoint the error in this diagrammatic calculation; numerical simulations in Sec. III D 5, however, seem to agree with our predictions.

${ }^{27}$ T. Hikihara, A. Furusaki, and M. Sigrist, Phys. Rev. B 60, 12116 (1999), implemented numerically a somewhat extended RG scheme; calculations (even if numerical) in this spirit could be used to further check validity of the basic RG procedures.

${ }^{28}$ This is in fact a rather generic feature of the first corrections to our leading-order results for dynamical properties, namely, that such corrections turn out to be closely related to ground-state correlators of the problem. A particularly explicit example of such a connection occurs in the free-fermion random-hopping problem (see Sec. VIA) where the leading correction to the wave function at energy $\epsilon$ (or equivalently, the leading correction to the Green function at frequency $\epsilon$ ) is directly related to the statistics of the zero-energy wave function of the problem [D. S. Fisher (unpublished); O. Motrunich et al. (unpublished)].

${ }^{29}$ For the random transverse-field Ising chain, analysis in the spirit of Sec. III E and Ref. 6, Sec. VI A shows that higher-order corrections only renormalize the effective cluster moments entering the average dynamical response functions by numerical factors of order one. This only affects the values of some nonuniversal prefactors and has no effect on the asymptotic behavior of the correlators in the low-frequency long-time limit. More precisely, one can argue that the average dynamical response of a given spin $\sigma_{j}$ at low-frequency $\omega$ is dominated by instances when one of the $\sigma_{j}$ 's close neighbors (within few lattice spacings) is dynamically active at this frequency; these are precisely the situations captured by the leading-order result. Moreover, for the case of the autocorrelations of the boundary spin, it is easy to carry through an explicit calculation of the first correction using the results of Ref. 6, Sec. V B and Ref. 34. The result shows explicitly that only the nonuniversal prefactor acquires a correction, and the long-time behavior of the correlator is unchanged.

${ }^{30}$ Note, however, that Rule 4 of the RG procedure of Ref. 8 neglects next-nearest-neighbor bonds that are generated to the same order (Ref. 18). This error will affect the accuracy of the RG in the early stages but will cease to matter at low-energies. This is because the broad distributions that arise at low energies guarantee that the use of Rule 4 will almost always be immediately followed by an application of Rule 2, which will eliminate the next-nearest-neighbor couplings.

${ }^{31}$ Suppose that in the initial system, with spins labeled $i=1,2, \ldots$, the phase lengths $l_{1}, l_{2}, \ldots$, are all positive, and define $l_{0}=0$; we assign each initial spin $i$ a phase position $x_{i}$ and an identical geometrical position $d_{i}, d_{i}=x_{i}$, via $x_{1}=l_{0}, x_{2}=x_{1}+l_{1}$, etc. At each step of the RG, we keep labels of the remaining spins unchanged, and in the case (iii) assign to the newly created half-spin the label of one of its "parents" (it does not matter which one); as we run the RG, the phase positions of the remaining spins can then be referenced according to their labels relative to the (fixed) geometrical positions of the initial spins. The precise statement is: Let $j_{1}<j_{2}<\ldots$, label the remaining spins with the corresponding phase lengths $\widetilde{l}_{0}, \widetilde{l}_{1}, \widetilde{l}_{2}, \ldots$, and the phase positions $\tilde{x}\left(j_{1}\right)=\widetilde{l}_{0}, \tilde{x}\left(j_{2}\right)=\tilde{x}\left(j_{1}\right)+\tilde{l}_{1}$, etc.; there exists a sequence of intervening initial spins $\left\{a_{k}\right\}, a_{1} \leqslant j_{1} \leqslant a_{2} \leqslant j_{2}$ $\leqslant \ldots$, such that for each remaining spin $j_{k}$ we have $d\left(a_{k}\right)$ $-\left[d\left(a_{k+1}\right)-d\left(a_{k}\right)\right] \leqslant \tilde{x}\left(j_{k}\right) \leqslant d\left(a_{k+1}\right)+\left[d\left(a_{k+1}\right)-d\left(a_{k}\right)\right]$, that is, the remaining spins cannot deviate very far outside the corresponding bounding geometrical intervals. Thus, the phase positions of the remaining spins agree roughly with the geometrical positions that we would assign to them according to their labels.

${ }^{32}$ Z. Wang, M. P. A. Fisher, S. M. Girvin, and J. T. Chalker, Phys. Rev. B 61, 8326 (2000).

${ }^{33}$ L. J. Azevedo et al., Solid State Commun. 16, 1267 (1975).

${ }^{34}$ D. S. Fisher and A. P. Young, Phys. Rev. B 58, 9131 (1998).

${ }^{35}$ T. Senthil and S. Sachdev, Phys. Rev. Lett. 77, 5292 (1996).

${ }^{36}$ A. A. Gogolin and V. I. Melnikov, Zh. Éksp. Teor. Fiz. 73, 706 (1977) [Sov. Phys. JETP 46, 369 (1977)].

${ }^{37}$ I. M. Lifshits, S. A. Gredeskul, and L. A. Pastur, Introduction to the Theory of Disordered Systems (Wiley, New York, 1988). 